\title{
Synthesen von Organogalliumhydriden und Organogalliumhalogeniden
}

\author{
Dissertation \\ zur Erlangung des Doktorgrades \\ der Mathematisch-Naturwissenschaftlichen Fakultäten \\ der Georg-August-Universität zu Göttingen
}

vorgelegt von

Hans-Jürgen Ahn

aus Erfurt

Göttingen 2005 
D7

Referent:

Prof. Dr. Dr. h.c. mult. H. W. Roesky

Korreferent:

Priv. Doz. Dr. N. Mösch-Zanetti

Tag der mündlichen Prüfung: $\quad$ 28.06.2005 
Die vorliegende Dissertation wurde in der Zeit von Mai 2000 bis Mai 2005 unter Anleitung von Prof. H. W. Roesky am Institut für Anorganische Chemie der GeorgAugust-Universität zu Göttingen angefertigt.

Meinem verehrten Lehrer,

\section{Herrn Prof. Dr. Dr. h. c. mult. H. W. Roesky}

danke ich sehr herzlich für die interessante Themenstellung, die ausgezeichneten Arbeitsbedingungen, sowie sein stetiges Interesse am Fortgang dieser Arbeit und seine freundliche Betreuung.

Für die Durchführung der Einkristallröntgenstrukturanalysen danke ich den Herren Dr. M. Noltemeyer, H.-G. Schmidt und Prof. Dr. J. Magull. Für die Durchführung der spektroskopischen und analytischen Arbeiten gilt mein Dank den Damen und Herren Dr. G. Elter, R. Schöne, W. Zolke, Dr. V. Jancik und S. Singh (NMR), T. Schuchardt, A. Rehbein und J. Schöne (MS), sowie Dipl.-Chem. H. Rusev, D. Kumpert, S. Petrich, C. Wittenberg und A. Wraage (Elementaranalysen). Herrn J. Schimkoviak und M. Schlote danke ich für die Bereitstellung des Materials und der Chemikalien. Allen meinen Kolleginnen und Kollegen des Instituts danke ich für die gute Zusammenarbeit und das nette Arbeitsklima. Besonders freundlicher Dank gebührt

Dr. C. Ackerhans, T. Blunck, Dr. M. Gorol, Dr. V. Jancik, Dr. J. Janssen, Dr. S.S. Kumar, Dr. D. Reddy, S. Singh, Dr. A. Stasch und Dr. M. Witt.

Ein ganz besonderes Dankeschön geht an meine Ehefrau Soo-Hyun Ahn für die liebeund verständnisvolle Unterstützung beim Entstehen dieser Arbeit. 


\section{Inhaltsverzeichnis}

Inhaltsverzeichnis

Abkürzungsverzeichnis $\quad$ VI

1. Einleitung 1

1.1 Anwendungen von Gallium in der modernen

Informationstechnologie 1

$\begin{array}{lll}1.2 & \text { Aluminium und Galliumhydride } & 3\end{array}$

2. Aufgabenstellung 5

3. Theoretischer Teil 6

$\begin{array}{lll}\text { 3.0 Modifizierte Darstellung von } \mathbf{G a H}_{3} \cdot \mathrm{NMe}_{3} & 6\end{array}$

3.1 Umsetzung von $\mathrm{GaH}_{3} \cdot \mathrm{NMe}_{3}$ mit Acetylenen $\quad 7$

3.1.1.1 Darstellung von $\mathrm{Me}_{3} \mathrm{~N} \cdot \mathrm{Ga}(-\mathrm{C} \equiv \mathrm{C}-\mathrm{Ph})_{3} \quad 8$

3.1.1.2 Diskussion der Einkristallröntgenstrukturanalyse von $\mathrm{Me}_{3} \mathrm{~N} \cdot \mathrm{Ga}(-\mathrm{C} \equiv \mathrm{C}-\mathrm{Ph})_{3}$

3.1.2.1 Darstellung von $\mathrm{Me}_{3} \mathrm{~N} \cdot \mathrm{Ga}(-\mathrm{C} \equiv \mathrm{C}-\mathrm{TMS})_{3}$

3.1.2.2 Diskussion der Einkristallröntgenstrukturanalyse von $\mathrm{Me}_{3} \mathrm{~N} \cdot \mathrm{Ga}(-\mathrm{C} \equiv \mathrm{C}-\mathrm{TMS})_{2} \mathrm{Cl}$

3.1.3 Darstellung von $\mathrm{Me}_{3} \mathrm{~N} \cdot \mathrm{Ga}(-\mathrm{C} \equiv \mathrm{C}-\mathrm{S}-n-\mathrm{Bu})_{3} \quad 16$

3.1.4 Darstellung von $\mathrm{Me}_{3} \mathrm{~N} \cdot \mathrm{Ga}\left(-\mathrm{C} \equiv \mathrm{C}-\mathrm{SO}_{2}-\left(p-\mathrm{MeC}_{6} \mathrm{H}_{4}\right)\right)_{3} \quad 16$

3.2 Austausch von $\mathrm{NMe}_{3}$ durch Arduengocarbene am $\mathbf{G a H}_{3} \quad 17$

3.2.1.1 Darstellung von $\mathrm{H}_{3} \mathrm{Ga} \cdot \mathrm{C}(\mathrm{N}(\text { iso-propyl }))_{2} \mathrm{C}_{2} \mathrm{H}_{2}$

3.2.1.2 Diskussion der Einkristallröntgenstrukturanalyse

von $\mathrm{H}_{3} \mathrm{Ga} \cdot \mathrm{C}(\mathrm{N}(\text { iso-propyl }))_{2} \mathrm{C}_{2} \mathrm{H}_{2}$ 
3.2.2 Darstellung von $\mathrm{H}_{3} \mathrm{Ga} \cdot \mathrm{C}(\mathrm{NMes}){ }_{2} \mathrm{C}_{2} \mathrm{H}_{2}$

3.3 Umsetzung von $\mathrm{GaH}_{3}$-Arduengocarbenadukt mit TMSI

3.3.1.1 Umsetzung von $\mathrm{H}_{3} \mathrm{Ga} \cdot \mathrm{C}(\mathrm{NMes})_{2} \mathrm{C}_{2} \mathrm{H}_{2}$ mit 1,2 und 3 Äq. TMSI

3.3.1.2 Diskussion der Einkristallröntgenstrukturanalyse von $\mathrm{I}_{3} \mathrm{Ga} \cdot \mathrm{C}(\mathrm{NMes})_{2} \mathrm{C}_{2} \mathrm{H}_{2}$

3.4 Darstellung von (Dipp) 2 NacNacGa-Halogeniden und Hydriden 24

3.4.1.1 Darstellung von (Dipp) ${ }_{2} \mathrm{NacNacGaBr}_{2}$

3.4.1.2 Diskussion der Einkristallröntgenstrukturanalyse von (Dipp) ${ }_{2} \mathrm{NacNacGaBr}_{2}$

3.4.2.1 Darstellung von (Dipp) ${ }_{2} \mathrm{NacNacGaF}_{2}$

3.4.2.2 Diskussion der Einkristallröntgenstrukturanalyse von (Dipp) ${ }_{2} \mathrm{NacNacGaF}_{2}$

3.4.3.1 Darstellung von (Dipp) ${ }_{2} \mathrm{NacNacGaH}_{2}$ aus (Dipp) ${ }_{2} \mathrm{NacNacGaI}_{2}$ oder (Dipp) ${ }_{2} \mathrm{NacNacGaCl}_{2}$ und Superhydrid

3.4.3.2 Diskussion der Einkristallröntgenstrukturanalyse von $(\text { Dipp })_{2} \mathrm{NacNacGaH}_{2}$

3.4.4 Darstellung von (Dipp) ${ }_{2} \mathrm{NacNacGaHCl}$

3.5 Untersuchung der Reaktivität von (Dipp) ${ }_{2} \mathrm{NacNacGaH}_{2}$ gegenüber unterschiedlichen Funktionalitäten

3.5.1 Umsetzung von (Dipp) ${ }_{2} \mathrm{NacNacGaH}_{2}$ mit $\mathrm{H}-\mathrm{C} \equiv \mathrm{C}-\mathrm{Ph} \quad 40$

3.5.2 Umsetzung von (Dipp) ${ }_{2} \mathrm{NacNacGaH}_{2}$ mit 1,2-cis-Diphenylethen 41

3.5.3 Umsetzung von (Dipp) ${ }_{2} \mathrm{NacNacGaH}_{2}$ mit ICN 41

3.5.4 Umsetzung von (Dipp) ${ }_{2} \mathrm{NacNacGaH}_{2}$ mit Benzonitril 41

3.5.5 Umsetzung von (Dipp) ${ }_{2} \mathrm{NacNacGaH}_{2}$ mit Methylhydrazin 42 
3.5.6 Umsetzung von (Dipp) ${ }_{2} \mathrm{NacNacGaH}_{2}$ mit tert-Butylhydroperoxid

3.5.7 Umsetzung von (Dipp) ${ }_{2} \mathrm{NacNacGaH}_{2}$ mit Trifluorethanol

3.6 Umsetzung von (Dipp) ${ }_{2} \mathrm{NacNacGaH}_{2}$ mit Säuren

3.6.1 Umsetzung von (Dipp) ${ }_{2} \mathrm{NacNacGaH}_{2}$ mit Benzoesäure

3.6.2 Umsetzung von (Dipp) ${ }_{2} \mathrm{NacNacGaH}_{2}$ mit Essigsäure

3.6.2.1 Umsetzung von (Dipp) ${ }_{2} \mathrm{NacNacGaH}_{2}$ mit Trifluoressigsäure

3.6.2.2 Diskussion der Einkristallröntgenstrukturanalyse

von $\left[(\mathrm{Dipp})_{2} \mathrm{NacNacH}_{2}\right]^{+}\left[\mathrm{OOCCF}_{3}\right]^{-}$

4. Experimenteller Teil

4.0 Allgemeine Arbeitstechniken, Meßmethoden und Darstellung $\begin{array}{ll}\text { der Ausgangsverbindungen } & 48\end{array}$

$\begin{array}{lll}\text { 4.0.1 Allgemeine Arbeitstechniken } & 48\end{array}$

$\begin{array}{lll}\text { 4.0.2 Meßmethoden } & 48\end{array}$

$\begin{array}{lll}\text { 4.0.3 Darstellung der Ausgangsverbindungen } & 50\end{array}$

4.0.3.1 Modifizierte Darstellung von $\mathrm{GaH}_{3} \cdot \mathrm{NMe}_{3} \quad 50$

4.1 Umsetzung von $\mathrm{GaH}_{3} \cdot \mathrm{NMe}_{3}$ mit Acetylenen 52

4.1.1 Darstellung von $\mathrm{Me}_{3} \mathrm{~N} \cdot \mathrm{Ga}(-\mathrm{C} \equiv \mathrm{C}-\mathrm{Ph})_{3}$

4.1.2 Darstellung von $\mathrm{Me}_{3} \mathrm{~N} \cdot \mathrm{Ga}(-\mathrm{C} \equiv \mathrm{C}-\mathrm{TMS})_{3}$

4.1.3 Darstellung von $\mathrm{Me}_{3} \mathrm{~N} \cdot \mathrm{Ga}(-\mathrm{C} \equiv \mathrm{C}-\mathrm{S}-n-\mathrm{Bu})_{3}$

4.1.4 Darstellung von $\mathrm{NMe}_{3} \cdot \mathrm{Ga}\left(-\mathrm{C} \equiv \mathrm{C}-\mathrm{SO}_{2}-\left(p-\mathrm{MeC}_{6} \mathrm{H}_{4}\right)\right)_{3}$

4.2 Austausch von $\mathrm{NMe}_{3}$ durch Arduengocarbene am $\mathrm{GaH}_{3}$

4.2.1 Darstellung von $\mathrm{H}_{3} \mathrm{Ga} \cdot \mathrm{C}(\mathrm{N}(\text { iso-propyl }))_{2} \mathrm{C}_{2} \mathrm{H}_{2}$

4.2.2 Darstellung von $\mathrm{H}_{3} \mathrm{Ga} \cdot \mathrm{C}(\mathrm{NMes})_{2} \mathrm{C}_{2} \mathrm{H}_{2}$ 
4.3 Umsetzung von $\mathrm{GaH}_{3}$-Arduengocarbenadukt mit TMSI

4.3.1 Umsetzung von $\mathrm{H}_{3} \mathrm{Ga} \cdot \mathrm{C}(\mathrm{NMes})_{2} \mathrm{C}_{2} \mathrm{H}_{2}$ mit 1,2 und 3 Äq. TMSI

4.4 Darstellung von (Dipp) ${ }_{2}$ NacNacGa-Halogeniden und Hydriden 58

4.4.1 Darstellung von (Dipp) ${ }_{2} \mathrm{NacNacGaBr}_{2} \quad 58$

4.4.2 Darstellung von (Dipp) ${ }_{2} \mathrm{NacNacGaF}_{2} \quad 59$

4.4.3.a Darstellung von (Dipp) ${ }_{2} \mathrm{NacNacGaH}_{2}$ aus (Dipp) ${ }_{2} \mathrm{NacNacGaI}_{2}$ und Superhydrid

4.4.3.b Darstellung von (Dipp) ${ }_{2} \mathrm{NacNacGaH}_{2}$ aus (Dipp) ${ }_{2} \mathrm{NacNacGaCl}_{2}$ und Superhydrid

4.4.4 Darstellung von (Dipp) ${ }_{2} \mathrm{NacNacGaHCl}$

4.5 Untersuchung der Reaktivität von (Dipp) ${ }_{2} \mathrm{NacNacGaH}_{2}$ gegenüber unterschiedlichen Funktionalitäten

4.5.1 Umsetzung von (Dipp) ${ }_{2} \mathrm{NacNacGaH}_{2}$ mit $\mathrm{H}-\mathrm{C} \equiv \mathrm{C}-\mathrm{Ph}$

4.5.2 Umsetzung von (Dipp) ${ }_{2} \mathrm{NacNacGaH}_{2}$ mit 1,2-cis-Diphenylethen

4.5.3 Umsetzung von (Dipp) ${ }_{2} \mathrm{NacNacGaH}_{2}$ mit ICN 65

4.5.4 Umsetzung von (Dipp) ${ }_{2} \mathrm{NacNacGaH}_{2}$ mit Benzonitril 66

4.5.5 Umsetzung von (Dipp) ${ }_{2} \mathrm{NacNacGaH}_{2}$ mit Methylhydrazin 67

4.5.6 Umsetzung von (Dipp) ${ }_{2} \mathrm{NacNacGaH}_{2}$ mit tert-Butylhydroperoxid 67

4.5.7 Umsetzung von (Dipp) ${ }_{2} \mathrm{NacNacGaH}_{2}$ mit Trifluorethanol 68

4.6 Umsetzung von (Dipp) ${ }_{2} \mathrm{NacNacGaH}_{2}$ mit Säuren 68

4.6.1 Umsetzung von (Dipp) ${ }_{2} \mathrm{NacNacGaH}_{2}$ mit Benzoesäure 68

4.6.2 Umsetzung von (Dipp) ${ }_{2} \mathrm{NacNacGaH}_{2}$ mit Essigsäure 69

4.6.3 Umsetzung von (Dipp) ${ }_{2} \mathrm{NacNacGaH}_{2}$ mit Trifluoressigsäure 71

$\begin{array}{ll}\text { 5. Ergebnisse } & 72\end{array}$ 
6. Behandlung und Entsorgung der Abfälle 74

$\begin{array}{ll}\text { 7. Röntgenographischer Anhang } & 77\end{array}$

$\begin{array}{lll}\text { 7.1 } & \mathrm{Me}_{3} \mathbf{N} \cdot \mathbf{G a}(\mathrm{C} \equiv \mathrm{C}-\mathrm{Ph})_{3} & 75\end{array}$

$\begin{array}{lll}\text { 7.2 } & \mathrm{Me}_{3} \mathrm{~N} \cdot \mathrm{Ga}(\mathrm{C} \equiv \mathrm{C}-\mathrm{TMS})_{2} \mathrm{Cl} & 76\end{array}$

$\begin{array}{lll}7.3 & \mathbf{H}_{3} \mathbf{G a} \cdot \mathbf{C}(\mathbf{N}(\text { iso-propyl }))_{2} \mathbf{C}_{2} \mathbf{H}_{2} & 77\end{array}$

$\begin{array}{lll}7.4 & \mathrm{I}_{3} \mathrm{Ga} \cdot \mathrm{C}(\mathrm{NMes})_{2} \mathrm{C}_{2} \mathrm{H}_{2} & 78\end{array}$

$\begin{array}{lll}7.5 & (\text { Dipp })_{2} \mathrm{NacNacGaBr}_{2} & 79\end{array}$

$\begin{array}{llr}\text { 7.6 (Dipp) }{ }_{2} \mathrm{NacNacGaF}_{2} & 80\end{array}$

$\begin{array}{lll}7.7 & (\text { Dipp })_{2} \mathrm{NacNacGaH}_{2} & 81\end{array}$

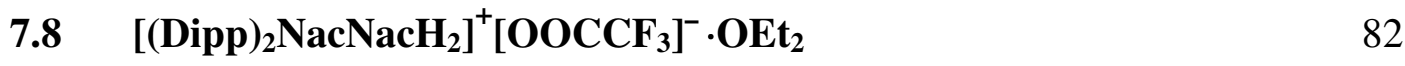

8. Literaturverzeichnis 83 


\section{Abkürzungsverzeichnis}

\begin{tabular}{|c|c|}
\hline$\AA$ & Ångström \\
\hline Äq. & Äquivalent \\
\hline Ar & Aryl \\
\hline ber. & berechnet \\
\hline br & breites Signal \\
\hline $\mathrm{Bu}$ & Buthyl \\
\hline bzw. & beziehungsweise \\
\hline $\mathrm{d}$ & $\operatorname{Tag}(\mathrm{e})$ \\
\hline$\delta$ & chemische Verschiebung \\
\hline Dipp & 2,6-Di-iso-propylphenyl \\
\hline \multirow[t]{2}{*}{$(\mathrm{Dipp})_{2} \mathrm{NacNac}$} & [N,N'-Bis(2,6-di-iso-propylphenyl)pentan-2,4-diiminato- \\
\hline & $\left.\mathrm{N}, \mathrm{N}^{\prime}\right]$-Substituent \\
\hline EI & Elektronenstoss-Ionisation \\
\hline Et & Ethyl \\
\hline gef. & gefunden \\
\hline${ }^{\circ} \mathrm{C}$ & Grad Celsius \\
\hline $\mathrm{h}$ & Stunde(n) \\
\hline $\mathrm{Hz}$ & Hertz \\
\hline iso & verzweigt \\
\hline IR & Infrarot \\
\hline$J$ & Kopplungskonstante \\
\hline $\mathrm{K}$ & Kelvin \\
\hline
\end{tabular}


$\lambda$

Frequenz

$m$

meta

$\mathrm{m}$

Meter

M

Molekülion

$\mathrm{Me}$

Methyl

$\mathrm{m} / \mathrm{e}$

Masse-Ladungsverhältnis

Mes

Mesityl

$\mathrm{Mg}$

Megagramm

$\mathrm{MHz}$

Megahertz

$\mathrm{mm}$

Millimeter

MS

Massenspektrum

$n$

unverzweigt

nm

Nanometer

NMR

kernmagnetische Resonanz

$o$

ortho

$p$

para

$\mathrm{Ph}$

Phenyl

pm

Pikometer

ppm

parts per million

$\mathrm{R}$

organischer Rest

THF

Tetrahydrofuran

TMS

Trimethylsilyl

$\tilde{v}$

Wellenzahl 
Kristalls 


\section{1. $\quad$ Einleitung}

\subsection{Anwendungen von Gallium in der modernen Informations- technologie ${ }^{[1,2]}$}

Gallium spielt in der modernen Informationstechnologie eine zunehmende Rolle. In der Grundlagenforschung und der Halbleiterindustrie wird beispielsweise Galliumarsenid vor allem im Rahmen des Materialsystems Aluminium-GalliumArsenid zur Herstellung von Halbleiterheterostrukturen verwendet. Durch daraus herstellbare, schnell schaltende Bauteile - zehnmal schneller als solche aus Silicium die zudem noch weitaus weniger Störungen in analogen Signale einbringen und einen geringeren Energiebedarf aufweisen als Silicium-Bauteile, gilt Galliumarsenid als wichtiger Grundstoff für die Telekommunikation. In leistungsfähigen Mobiltelefonen basieren die Bauelemente (Integrierte Schaltkreise) für den Empfang und das Senden von Signalen auf Galliumarsenid. Darüber hinaus wird Galliumarsenid benutzt, um mit Hilfe von Lasern bzw. oberflächenemittierenden Lasern (VCSEL) Informationen durch Glasfasernetze zu senden sowie Satelliten mit Energie aus hochspezialisierten Solarzellen (Photovoltaik) zu versorgen. Selbst im Alltag kommt Galliumarsenid zur Anwendung, wie etwa beim Abspielen einer CD, wo ein Laser aus Galliumarsenid zum Musikgenuss verhilft. Ein weiteres wichtiges Produkt sind Leuchtdioden. Dennoch hat Galliumarsenid das Silicium als Massen-Halbleiter für eher alltägliche Anwendungen nicht verdrängen können. Die Gründe dafür sind der geringere Preis von Silicium, dessen größere Einkristalle und die Möglichkeit, in Silicium-Strukturen 
leichter sehr gute Isolator-Bereiche aus Quarz (Siliciumdioxid) zu erzeugen. Dazu kommt die völlige Ungiftigkeit von Silicium im Gegensatz zum gesundheitlich sehr problematischen Arsen. Während Galliumarsenid bei den Verbindungshalbleitern nach wie vor das dominierende Substratmaterial für die Herstellung integrierter Höchstfrequenzschaltkreise ist, gewinnen für sehr große Datenübertragungsraten Indiumphosphid und für Leistungsanwendungen die sogenannten "Wide Band Gap" (WBG) - Halbleiter (Siliciumcarbid und Galliumnitrid) an Bedeutung. Im Zuge der immer höheren Anforderungen an die Datenübertragungsraten (Fernziel 160 Gbit/s) sind FuE-Aktivitäten für neue Komponenten und Technologien in der Hochgeschwindigkeitselektronik unumgänglich. Das Material der Wahl ist hier Indiumphosphid (InP). Nur dieser Halbleiter ist in der Lage, die gewünschte Performance in der Signalverarbeitung und in der "High Speed Elektronik" zu erreichen. Die entsprechenden Forschungsaktivitäten konzentrieren sich daher auf den Gebieten InP-Substratentwicklung und Bauelement- und Schaltkreisherstellung auf InP-Basis. Die wissenschaftlich-technische Entwicklung auf dem Gebiet der InPTechnologie wurde vom BMBF im Rahmen der institutionellen Förderung schon frühzeitig aufgegriffen und forciert. Die jetzt geplanten Fördermaßnahmen basieren auf dem dadurch geschaffenen Grundlagenwissen. Insbesondere für den erheblich gestiegenen Leistungsbedarf der Systeme in der mobilen Kommunikationstechnik sind neue Komponenten gefragt. Hier bieten die WBG - Halbleiter auf Grund ihrer physikalischen Eigenschaften (große Bandlücke) ein enormes Potenzial. Sie können bei hohen Spannungen und sehr hohen Frequenzen betrieben werden, sie verfügen über große Stromdichten und hohe Ladungsträgergeschwindigkeiten. Bei gleichzeitiger Reduzierung der Bauelementeabmessungen lassen sich somit sehr hohe 
Leistungsdichten erzielen, so dass Bauelemente auf GaN- bzw. SiC-Basis prädestiniert sind für robuste und extrem leistungsstarke Verstärker. (z.B. in Mobilfunk-Basisstationen, in der Satelliten- und Telekommunikation). Für leistungsfähige Lichtquellen und Laser insbesondere im Bereich Blau und Ultraviolett wird ebenfalls das Material Galliumnitrid $(\mathrm{GaN})$ benötigt. Die im blauen Spektralbereich emittierenden Laserdioden sowie die Mehrschicht-Disktechnologien gelten als Meilensteine zur Steigerung der Datenspeicherkapazität von optischen Speicherplatten.

\subsection{Aluminium und Galliumhydride}

$\mathrm{AlH}_{3}$ wurde 1966 das erste mal von E.C. Ashby ${ }^{[3]}$ dargestellt. Dabei handelt es sich um einen hochpolymeren weißen bis $\mathrm{zu} 100{ }^{\circ} \mathrm{C}$ thermisch beständigen Feststoff. Jedes Aluminiumatom ist dabei von 6 Wasserstoffen umgeben. Analog zum Aluminiumhydrid wurde $1971\left(\mathrm{GaH}_{3}\right)_{\mathrm{n}}$ dargestellt ${ }^{[4]}$. Dies lässt sich zum Unterschied zum Aluminiumhydrid als niedermolekulare viskose Flüssigkeit herstellen, die oberhalb von $-15^{\circ} \mathrm{C}$ thermisch instabil ist. Der lewissaure Charakter der Verbindung führt aber auch hier nach kurzer Zeit zu einer vollständigen Polymerisation. Die monomeren Hydride lassen sich nur durch Adduktbildung mit Lewis-Basen wie Ether, Amine oder Phosphane gewinnen. Obwohl dreiwertiges Gallium eine große Analogie zum Aluminium zeigt, besteht hier ein deutlicher Unterschied. Wird ein Aluminiumhydridetherat auf $100^{\circ} \mathrm{C}$ erhitzt, bildet sich festes, polymeres $\left(\mathrm{AlH}_{3}\right)_{\mathrm{n}}$. Das bedeutet, dass die koordinative Bindung zwischen Aluminium und, in dem Fall 
Sauerstoff, schwächer ist als die Al-H-Bindung. Monomere Aluminiumhydridaddukte sind demnach für Reaktionen bei höheren Temperaturen geeignet. Galliumhydridetherate zersetzen sich beim Erhitzen in elementares $\mathrm{Ga}$ und $\mathrm{H}_{2}$. Das kann einerseits bedeuten, dass die Ga-H-Bindung schwächer ist, als die koordinative Bindung. Andererseits ist es wahrscheinlicher, dass das monomer gebildete „freie“ $\mathrm{GaH}_{3}$ so instabil ist, dass der Zerfall viel schneller verläuft als die Polymerisation. Monomere Galliumhydridaddukte sind demnach viel schlechter bei hohen Temperaturen einsetzbar als analoge Aluminiumverbindungen.

Die Metall-Wasserstoff-Bindung beim Aluminiumhydrid ist stärker polarisiert als beim Galliumhydrid, die negative Partialladung am Hydridwasserstoff ist also höher. Das führt zu unterschiedlichen Reaktivitäten. 


\section{Aufgabenstellung}

Die Aufgabe besteht darin unterschiedliche Galliumhydride darzustellen, mit dem Ziel eine höhere thermische Belastbarkeit zu erreichen, um diese einer größeren Vielzahl von Reaktionsmöglichkeiten zugänglich $\mathrm{zu}$ machen, als es Galliumhydridtrimethylamin zulässt. Weiterhin soll untersucht werden inwieweit Organogalliumhydridhalogenide bzw. Organogalliumhalogenide darstellbar sind. 


\section{Theoretischer Teil}

\subsection{Modifizierte Darstellung von $\mathrm{GaH}_{3} \cdot \mathrm{NMe}_{3}$}

Die Darstellung von $\mathrm{GaH}_{3} \cdot \mathrm{NMe}_{3}$ ist zweistufig. Zuerst wurde $\mathrm{LiH}$ mit $\mathrm{GaCl}_{3}$ in Diethylether zu $\mathrm{LiGaH}_{4}$ umgesetzt. ${ }^{[5]} \mathrm{Da} \mathrm{LiH}$ in Diethylether unlöslich ist, wurde ein vierfacher Überschuss eingesetzt. Da LiH nur an der Oberfläche reagiert, war es notwendig diese zu vergrößern. Dazu wurde das $\mathrm{LiH}$ in einer Kugelmühle $2 \mathrm{~h}$ lang gemahlen. Dadurch wurde auch eine eventuell vorhandene passivierende $\mathrm{LiOH}-$ Schicht zerstört und die Reaktivität enorm gesteigert. Das erhaltene $\mathrm{LiGaH}_{4}$ musste nach Beendigung der Synthese in Diethylether gelöst bleiben, da sich nach dem Entfernen des Lösungsmittels das Produkt zersetzt hätte. Die Ausbeute wurde auf ca. 70-80 \% geschätzt. Diese Methode ist genauer als teilweise zersetztes $\mathrm{LiGaH}_{4} \mathrm{zu}$ wiegen. Im zweiten Schritt wurde das erhaltene $\mathrm{LiGaH}_{4}$ mit $\mathrm{NMe}_{3} \cdot \mathrm{HCl}$ solange umgesetzt bis kein $\mathrm{H}_{2}$ mehr entstand. ${ }^{[6]}$ Das bei der Reaktion entstandene LiCl brauchte nicht abfiltriert zu werden, da das Produkt sublimiert wurde. Um dies zu tun, musste erst das Lösungsmittel entfernt werden. $\mathrm{Da} \mathrm{GaH}_{3} \cdot \mathrm{NMe}_{3}$ leicht flüchtig ist, wurde der Diethylether bei $-45^{\circ} \mathrm{C}$ abdestilliert. Danach wurde das Produkt bei Raumtemperatur in ein spezielles Schlenkgefäss, welches auf $-45^{\circ} \mathrm{C}$ gekühlt war, sublimiert. Dabei blieben chlorhaltige Zwischenprodukte und $\mathrm{LiCl}$ zurück. $\mathrm{GaH}_{3} \cdot \mathrm{NMe}_{3}$ ist bei Raumtemperatur nicht beständig und muss bei $-27^{\circ} \mathrm{C}$ gelagert werden. 


\subsection{Umsetzung von $\mathrm{GaH}_{3} \cdot \mathrm{NMe}_{3}$ mit Acetylenen}

$\mathrm{GaH}_{3} \cdot \mathrm{NMe}_{3}$ reagiert unter Wasserstoffbildung mit Acetylenen. Die Ölbadtemperatur darf $55^{\circ} \mathrm{C}$ nicht übersteigen, da sonst Zersetzung in Gallium und Wasserstoff eintritt. Die Zersetzungsgeschwindigkeit ist dabei viel höher als die Reaktionsgeschwindigkeit, wodurch die Ausbeute drastisch reduziert wird. Die thermische Empfindlichkeit des Galliumhydrids grenzt die Reaktionsvielfalt im Vergleich zum Aluminiumhydrid stark ein. $\mathrm{AlH}_{3} \cdot \mathrm{NMe}_{3}$ bildet beispielsweise mit einem Unterschuss an Acetylenen ${ }^{[7,8,9]}$ oder Nitrilen $^{[10]}$ in siedendem Toluol innerhalb einer Stunde Cluster. Analoge Versuche mit Galliumhydrid scheiterten. Die Reaktionsgeschwindigkeit ist von der Acidität des Acetylens abhängig. Elektronenziehende Substituenten erhöhen hierbei die Reaktionsfreudigkeit.

$\mathrm{TMS}<\mathrm{S}-\mathrm{Bu}<\mathrm{Ph}<\mathrm{SO}_{2}-\left(p-\mathrm{MeC}_{6} \mathrm{H}_{4}\right)$

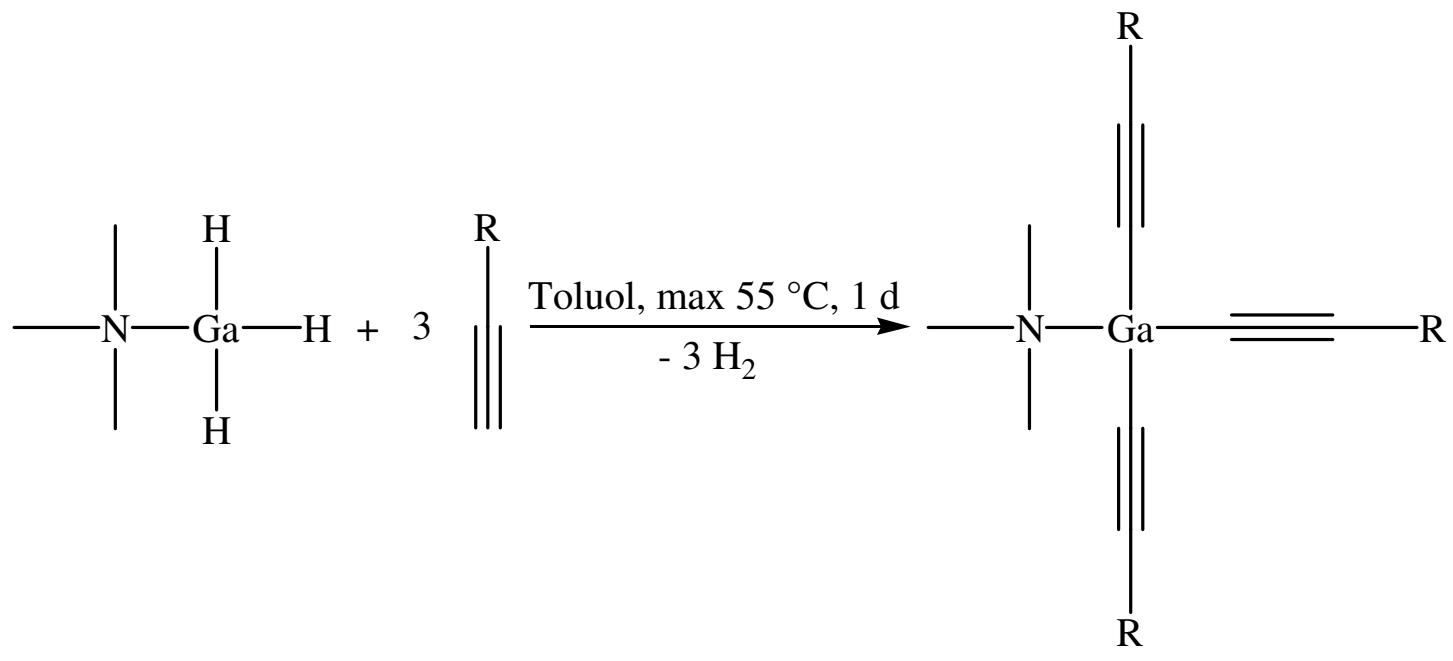

$\mathrm{R}=\mathrm{Ph}, \mathrm{TMS}, \mathrm{S}-\mathrm{Bu}, \mathrm{SO}_{2}-\left(p-\mathrm{MeC}_{6} \mathrm{H}_{4}\right)$

\section{Reaktionsgleichung 3.1}




\subsubsection{Darstellung von $\mathrm{Me}_{3} \mathrm{~N} \cdot \mathrm{Ga}(-\mathrm{C} \equiv \mathrm{C}-\mathrm{Ph})_{3}$}

Zur Herstellung von $\mathrm{Me}_{3} \mathrm{~N} \cdot \mathrm{Ga}(-\mathrm{C} \equiv \mathrm{C}-\mathrm{Ph})_{3}$ wurde $\mathrm{GaH}_{3} \cdot \mathrm{NMe}_{3}$ mit 10 Äquivalenten Phenylacetylen bei $55^{\circ} \mathrm{C}$ in Toluol umgesetzt. Der Überschuss an Phenylacetylen konnte nach Beendigung der Reaktion mit dem Lösungsmittel destillativ entfernt werden. Nach Kristallisation in Toluol wurden farblose Kristalle mit einem Schmelzpunkt von $151^{\circ} \mathrm{C}$ erhalten, die für eine Einkristallröntgenstrukturanalyse geeignet waren. Die Analysen ergaben:

Das EI-Massenspektrum zeigt einen Molekülionenpeak bei m/e von 431 mit einer 30\%igen Intensität. Durch Abspaltung der Trimetylamingruppe erscheint ein weiteres Signal bei m/e von 372 mit einer Intensität von $100 \%$.

Das IR-Spektrum zeigt zwei starke Absorptionsbanden bei Wellenzahlen von 2137 und $1210 \mathrm{~cm}^{-1}$.

Das ${ }^{1}$ H-NMR-Spektrum zeigt erwartungsgemäß ein Singulett bei 2.28 ppm für die Protonen der Aminfunktion und zwei Multipletts im aromatischen Bereich für die Phenylprotonen.

Im ${ }^{13}$ C-NMR-Spektrum kann eindeutig das breite Signal bei einer chemischen Verschiebung von 99.2 ppm den drei äquivalenten Kohlenstoffkernen, die direkt am Gallium gebunden sind, zugeordnet werden. Das Signal der Kohlenstoffkerne der Aminfunktion erscheinen bei 47.3 ppm. Ein schwaches (keine Protonen in der Nähe) aber scharfes Signal bei $107.2 \mathrm{ppm}$ ist dem nicht direkt am Gallium gebundenem Acetylenkohlenstoffkern zuzuordnen $(\mathrm{Ga}-\mathrm{C} \equiv C-\mathrm{Ph})$. Weitere vier Signale sind im aromatischen Bereich zwischen 125.1 und 132.3 ppm zu sehen. 


\subsubsection{Diskussion der Einkristallröntgenstrukturanalyse}

von $\mathrm{Me}_{3} \mathrm{~N} \cdot \mathrm{Ga}(-\mathrm{C} \equiv \mathrm{C}-\mathrm{Ph})_{3}$

Die strukturelle Untersuchung von $\mathrm{Me}_{3} \mathrm{~N} \cdot \mathrm{Ga}(-\mathrm{C} \equiv \mathrm{C}-\mathrm{Ph})_{3}$ bestätigt den angenommenen Aufbau des Moleküls. Bei der Reaktion mit Acetylenen werden alle hydridischen Wasserstoffe durch den organischen Substituenten bei Erhaltung der Dreifachbindung ersetzt.

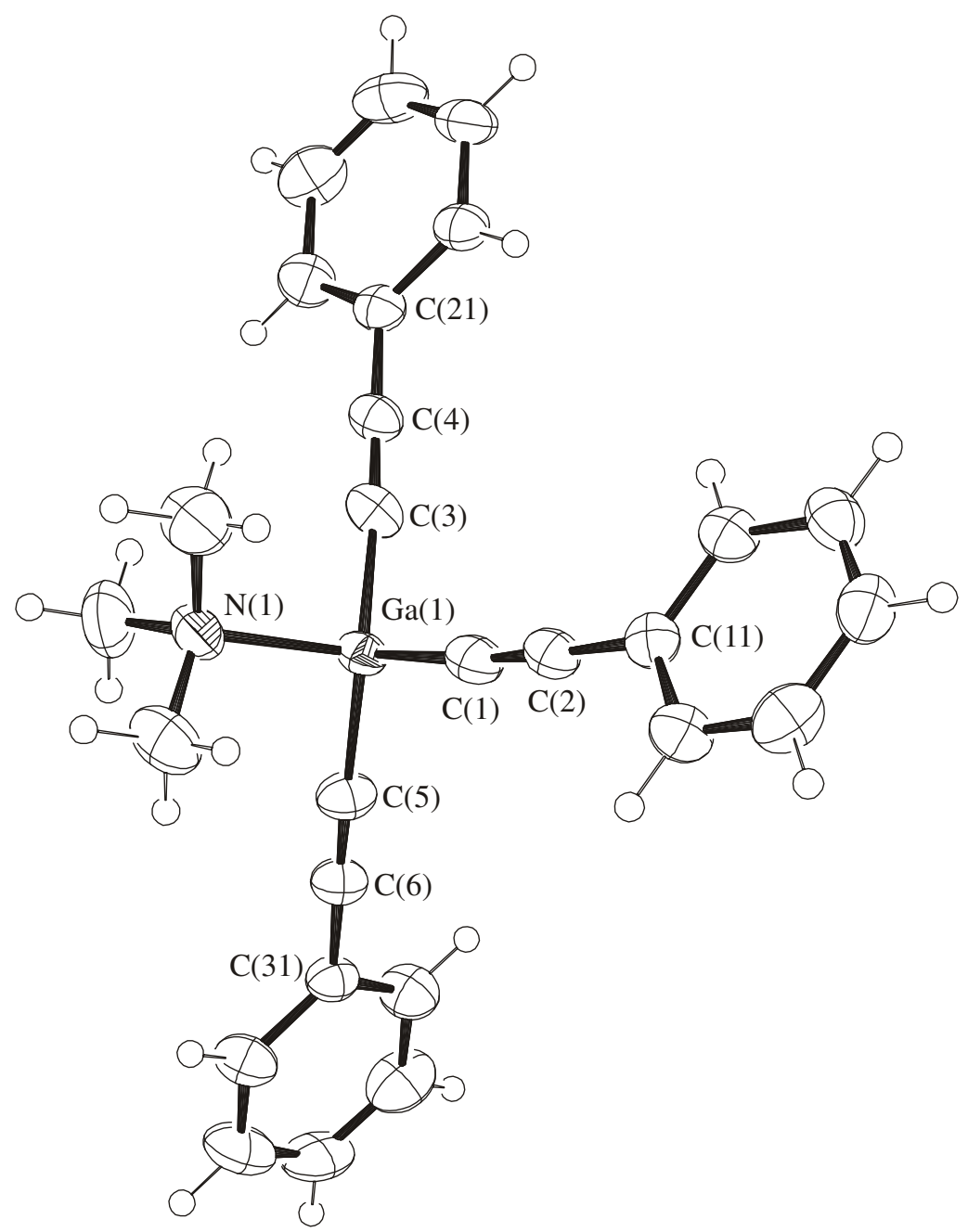

Abbildung 3.1.1.2: Struktur von $\mathrm{Me}_{3} \mathrm{~N} \cdot \mathrm{Ga}(-\mathrm{C} \equiv \mathrm{C}-\mathrm{Ph})_{3}$ im Kristall 
Tabelle 3.1.1.2: Ausgewählte Bindungslängen [pm] und Winkel $\left[^{\circ}\right]$ von $\mathrm{Me}_{3} \mathrm{~N} \cdot \mathrm{Ga}(-\mathrm{C} \equiv \mathrm{C}-\mathrm{Ph})_{3}$

$\begin{array}{llll}\mathrm{Ga}(1)-\mathrm{C}(5) & 192.9(4) & \mathrm{Ga}(1)-\mathrm{C}(1) & 194.0(4) \\ \mathrm{Ga}(1)-\mathrm{C}(3) & 194.0(4) & \mathrm{Ga}(1)-\mathrm{N}(1) & 203.4(4) \\ \mathrm{C}(1)-\mathrm{C}(2) & 120.2(5) & \mathrm{C}(3)-\mathrm{C}(4) & 120.7(5) \\ \mathrm{C}(5)-\mathrm{C}(6) & 119.8(6) & \mathrm{C}(2)-\mathrm{C}(11) & 143.3(5) \\ \mathrm{C}(4)-\mathrm{C}(21) & 143.5(6) & \mathrm{C}(6)-\mathrm{C}(31) & 143.8(6) \\ & & & \\ \mathrm{C}(5)-\mathrm{Ga}(1)-\mathrm{C}(1) & 116.25(17) & \mathrm{C}(5)-\mathrm{Ga}(1)-\mathrm{C}(3) & 115.82(16) \\ \mathrm{C}(1)-\mathrm{Ga}(1)-\mathrm{C}(3) & 113.21(16) & \mathrm{C}(5)-\mathrm{Ga}(1)-\mathrm{N}(1) & 103.72(15) \\ \mathrm{C}(1)-\mathrm{Ga}(1)-\mathrm{N}(1) & 101.23(16) & \mathrm{C}(3)-\mathrm{Ga}(1)-\mathrm{N}(1) & 104.01(16) \\ \mathrm{C}(4)-\mathrm{C}(3)-\mathrm{Ga}(1) & 173.8(3) & \mathrm{C}(6)-\mathrm{C}(5)-\mathrm{Ga}(1) & 178.6(4) \\ \mathrm{C}(1)-\mathrm{C}(2)-\mathrm{C}(11) & 178.1(4) & \mathrm{C}(3)-\mathrm{C}(4)-\mathrm{C}(21) & 176.1(4) \\ \mathrm{C}(5)-\mathrm{C}(6)-\mathrm{C}(31) & 177.7(4) & \end{array}$

Die Kristallisation erfolgte in der monoklinen Raumgruppe P2(1)/n. Die vier Substituenten sind verzerrt tetraedrisch um das zentrale Galliumatom angeordnet. Dabei sind die Acetylenfunktionen mit einem Bindungswinkel von $115^{\circ}$ sterisch anspruchsvoller als das Trimetylamin mit einem $\mathrm{C}-\mathrm{Ga}-\mathrm{N}-$ Winkel von $103^{\circ}$. Die Ga-N-Bindungslänge ist erwartungsgemäß mit 203.4 pm etwas länger als eine kovalente Ga-N-Einfachbindung (unter 200 pm), da es sich bei dem Amin um einen Donor handelt. Die Ga-C-Bindungen, mit einer durchschnittlichen Länge von 193 pm, liegen im, für diese Bindungsart, üblichen Bereich. Mit 120 pm für die 
Dreifachbindung der Acetylenfunktion ist bewiesen, dass es sich bei der Reaktion nicht um eine Hydrogallierung ${ }^{[11,12]}$ handelt, sondern das die Dreifachbindung erhalten bleibt. Die $\mathrm{Ga}-\mathrm{C} \equiv \mathrm{C}$ - und $\mathrm{C} \equiv \mathrm{C}-\mathrm{C}$-Winkel sind alle geringfügig kleiner als $180^{\circ}$, was auf eine Delokalisierung der $\pi$-Elektronen zurückzuführen ist.

\subsubsection{Darstellung von $\mathrm{Me}_{3} \mathrm{~N} \cdot \mathrm{Ga}(-\mathrm{C} \equiv \mathrm{C}-\mathrm{TMS})_{3}$}

Zur Herstellung von $\mathrm{NMe}_{3} \cdot \mathrm{Ga}(\mathrm{TMSacetylenid})_{3}$ wurde $\mathrm{GaH}_{3} \cdot \mathrm{NMe}_{3}$ mit 10 Äquivalenten Trimetylsilylacetylen bei $55^{\circ} \mathrm{C}$ in Toluol umgesetzt. Der Überschuss an Trimetylsilylacetylen konnte nach Beendigung der Reaktion mit dem Lösungsmittel destillativ entfernt werden. Die Ölbadtemperatur durfte $55^{\circ} \mathrm{C}$ nicht übersteigen, da sich sonst das Galliumhydrid zersetzt hätte. Die Zersetzungsgeschwindigkeit wäre dabei viel höher als die Reaktionsgeschwindigkeit, was die Ausbeute drastisch reduziert hätte. Strukturell untersucht werden konnte allerdings nur ein Nebenprodukt und zwar $\mathrm{Me}_{3} \mathrm{~N} \cdot \mathrm{GaCl}(\mathrm{TMSacetylenid})_{2}$. Dieses könnte auf zwei Wegen entstanden sein. Einerseits war möglicherweise das Galliumhydrid mit $\mathrm{GaH}_{2} \mathrm{Cl} \cdot \mathrm{NMe}_{3}$ verunreinigt. Andererseits könnte auch das Trimetylsilylacetylen mit TMSCl (wird bei der Herstellung von TMSacetylen

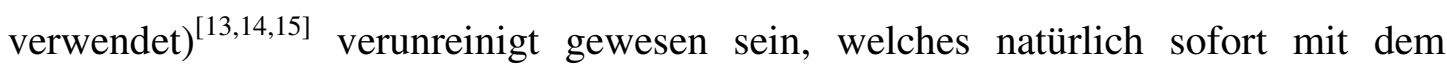
Galliumhydrid reagieren würde. Dieses strukturell nachgewiesene Nebenprodukt beweist allerdings die Reaktivität von Trimetylsilylacetylen gegenüber Galliumhydriden, da das Chlor am Galliumatom den hydridischen Charakter der Wasserstoffe vermindert und somit auch ihre Reaktivität. Die Ausbeute ist mit $44 \%$ im Vergleich zum Phenylderivat geringer, da der elektronenschiebende Effekt durch 
das Silicium die Acidität des Acetylens herabsetzt. NMR-spektroskopisch, massenspektrometrisch und elementaranalytisch wurde nur eine und zwar die Zielverbindung nachgewiesen. Für die Einkristallröntgenstrukturanalyse geeignete Kristalle wurden erst nach mehreren Kristallisationsversuchen und dann auch nur in einer sehr geringen Menge und Größe erhalten. Das chlorhaltige Derivat der Zielverbindung neigt durch seine polare Ga-Cl-Bindung viel stärker zur Kristallisation als die Zielverbindung und der eine vermessene Kristall ist dadurch nicht repräsentativ für die gesamte Synthese. Die analytischen Untersuchungen ergaben:

Das EI-Massenspektrum zeigt einen Molekülionenpeak bei m/e von 419 mit einer 9\%igen Intensität. Durch Abspaltung der Trimethylamingruppe erscheint ein weiteres Signal bei m/e von 359 mit einer Intensität von $16 \%$. Das Fragment, das durch die Abspaltung der Trimethylamingruppe und einer Methylgruppe entstand, erzeugte ein Peak bei m/e von 344 mit einer Intensität von $100 \%$. Das Signal einer chlorhaltigen Verbindung ist nicht vorhanden.

Das ${ }^{1}$ H-NMR-Spektrum zeigt erwartungsgemäß ein Singulett bei 1.96 ppm für die Protonen der Aminfunktion und ein Singulett bei einer chemischen Verschiebung von 0.12 ppm für die Trimethysilylprotonen. Im Bereich um $0.12 \mathrm{pm}$ sind mehrere leichte Verunreinigungen erkennbar. Bei ca. 2.05 ppm erscheint ein sehr kleines Signal, was auf das kristallisierte und röntgenographisch untersuchte Nebenprodukt hinweisen könnte. Die Intensität beträgt nur ca. 1-2\% der Protonen der Amingruppe des Hauptproduktes.

Im ${ }^{13}$ C-NMR-Spektrum kann die schwächste Resonanz bei einer chemischen Verschiebung von 89.7 ppm den drei äquivalenten Kohlenstoffkernen, die direkt am 
Gallium gebunden sind, zugeordnet werden. Das Signal der Kohlenstoffkerne der Aminfunktion erscheint deutlich bei $46.7 \mathrm{ppm}$. Ein weiteres Signal, was auf ein Nebenprodukt hinweist, ist in diesem Bereich nicht erkennbar. Ein schwaches Signal bei 93.9 ppm ist dem nicht direkt am Gallium gebundenem Acetylenkohlenstoffkern zuzuordnen $(\mathrm{Ga}-\mathrm{C} \equiv C-\mathrm{TMS})$. Das ${ }^{29} \mathrm{Si}$-NMR-Spektrum zeigt ein Signal bei einer chemischen Verschiebung von $-19.64 \mathrm{ppm}$, was dem ${ }^{29} \mathrm{Si}-\mathrm{Kern}$ der TMS-Gruppe zugeordnet werden kann.

\subsubsection{Diskussion der Einkristallröntgenstrukturanalyse von $\mathrm{Me}_{3} \mathrm{~N} \cdot \mathrm{Ga}(-\mathrm{C} \equiv \mathrm{C}-\mathrm{TMS})_{2} \mathrm{Cl}$}

Die Einkristallröntgenstrukturanalyse bestätigt die Reaktivität von TMSacetylen gegenüber den hydridischen Wasserstoffen des Galliumhydrids. Das Chloratom resultiert höchtwahrscheinlich durch die Verunreinigung des TMSacetylens mit TMSCl. 


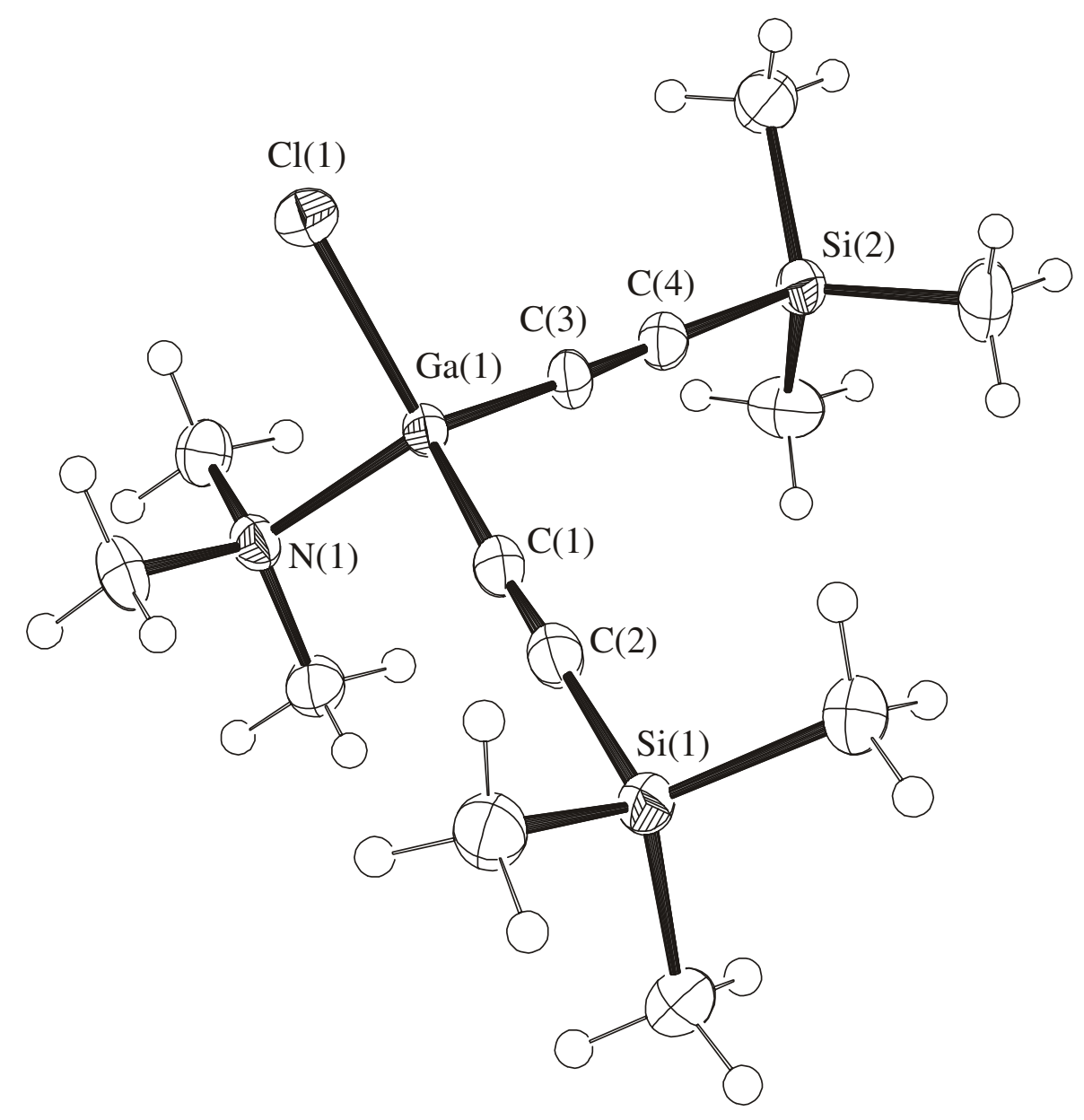

Abbildung 3.1.2.2: Struktur von $\mathrm{Me}_{3} \mathrm{~N} \cdot \mathrm{Ga}(-\mathrm{C} \equiv \mathrm{C}-\mathrm{TMS})_{2} \mathrm{Cl}$ im Kristall

Tabelle 3.1.2.2: Ausgewählte Bindungslängen [pm] und Winkel $\left[^{\circ}\right]$ von $\mathrm{Me}_{3} \mathrm{~N} \cdot \mathrm{Ga}(-\mathrm{C} \equiv \mathrm{C}-\mathrm{TMS})_{2} \mathrm{Cl}$

$\begin{array}{llll}\mathrm{Ga}(1)-\mathrm{C}(1) & 193.1(4) & \mathrm{G}(1)-\mathrm{C}(3) & 193 .(3) \\ \mathrm{Ga}(1)-\mathrm{N}(1) & 202.4(3) & \mathrm{Ga}(1)-\mathrm{Cl}(1) & 219.54(9) \\ \mathrm{Si}(1)-\mathrm{C}(2) & 184.5(4) & \mathrm{C}(1)-\mathrm{C}(2) & 120.5(5) \\ \mathrm{Si}(2)-\mathrm{C}(4) & 184.6(3) & \mathrm{C}(3)-\mathrm{C}(4) & 121.0(5)\end{array}$




$\begin{array}{llll}\mathrm{C}(1)-\mathrm{Ga}(1)-\mathrm{C}(3) & 116.56(14) & \mathrm{C}(1)-\mathrm{Ga}(1)-\mathrm{N}(1) & 106.27(13) \\ \mathrm{C}(3)-\mathrm{Ga}(1)-\mathrm{N}(1) & 107.69(12) & \mathrm{C}(1)-\mathrm{Ga}(1)-\mathrm{Cl}(1) & 112.73(10) \\ \mathrm{C}(3)-\mathrm{Ga}(1)-\mathrm{Cl}(1) & 110.28(11) & \mathrm{N}(1)-\mathrm{Ga}(1)-\mathrm{Cl}(1) & 102.07(8) \\ \mathrm{C}(2)-\mathrm{C}(1)-\mathrm{Ga}(1) & 176.7(3) & \mathrm{C}(1)-\mathrm{C}(2)-\mathrm{Si}(1) & 179.4(3) \\ \mathrm{C}(4)-\mathrm{C}(3)-\mathrm{Ga}(1) & 173.4(3) & \mathrm{C}(3)-\mathrm{C}(4)-\mathrm{Si}(2) & 179.2(3)\end{array}$

Die Kristallisation erfolgte in der triklinen Raumgruppe P-1. Die vier Substituenten sind verzerrt tetraedrisch um das zentrale Galliumatom angeordnet. Dabei sind die Acetylenfunktionen mit einem Bindungswinkel von $116^{\circ}$ sterisch am anspruchsvollsten. Der $\mathrm{Cl}-\mathrm{Ga}-\mathrm{N}-$ Winkel ist mit $102^{\circ}$ am kleinsten. Die $\mathrm{C}-\mathrm{Ga}-$ $\mathrm{N}-$ Winkel sind mit $107^{\circ}$ etwas kleiner als die $\mathrm{Cl}-\mathrm{Ga}-\mathrm{C}-$ Winkel mit $111^{\circ}$. Die Ga-N-Bindungslänge ist erwartungsgemäß mit 202.4 pm etwas länger als eine normale kovalente Ga-N-Bindung (unter 200 pm), da es sich bei dem Amin um eine Donor handelt. Sie ist aber ein pm kürzer als im chlorfreien Phenylacetylenderivat, was wahrscheinlich weniger an den TMS-Gruppen liegt, als am elektronenziehenden Chloratom. Die Ga-C-Bindungen, mit einer durchschnittlichen Länge von 193 pm, liegen im für diese Bindungsart üblichen Bereich. Mit $120 \mathrm{pm}$ für die Dreifachbindung der Acetylenfunktion ist bewiesen, das es sich bei der Reaktion nicht um eine Hydrogallierung ${ }^{[11,12]}$ handelt, sondern dass die Dreifachbindung erhalten bleibt. Die $\mathrm{Ga}-\mathrm{C} \equiv \mathrm{C}$ - und $\mathrm{C} \equiv \mathrm{C}-\mathrm{Si}$-Winkel sind alle geringfügig kleiner als $180^{\circ}$, was auf eine Delokalisierung der $\pi$-Elektronen zurückzuführen ist. 


\subsubsection{Darstellung von $\mathrm{Me}_{3} \mathrm{~N} \cdot \mathrm{Ga}(-\mathrm{C} \equiv \mathrm{C}-\mathrm{S}-n-\mathrm{Bu})_{3}$}

Zur Herstellung von $\mathrm{Me}_{3} \mathrm{~N} \cdot \mathrm{Ga}(-\mathrm{C} \equiv \mathrm{C}-\mathrm{S}-n-\mathrm{Bu})_{3}$ wurde $\mathrm{GaH}_{3} \cdot \mathrm{NMe}_{3}$ mit 3 Äquivalenten $\mathrm{H}-\mathrm{C} \equiv \mathrm{C}-\mathrm{S}-n-\mathrm{Bu}$ bei $55^{\circ} \mathrm{C}$ in Toluol umgesetzt. Nach dem Entfernen des Lösungsmittels wurde der Rückstand in Diethylether aufgenommen und filtriert. Es gelang nicht vom Produkt Einkristalle zu erhalten, da es sich um eine ölige Substanz handelt, die auch nicht bei tiefen Temperaturen kristallisiert. Die Analysen ergaben:

Das EI-Massenspektrum zeigt keinen Molekülionenpeak sondern nur Fragmente und zwar bei m/e von 393 mit einer Intensität von $2 \%$ für das Fragment, das durch Abspaltung der Trimethylamingruppe und einer Methylgruppe entstand. Durch Abspaltung der Amingruppe und von zwei Acetylfunktionen erscheint ein weiteres Signal bei m/e von 182 für $\mathrm{Ga}-\mathrm{C} \equiv \mathrm{C}-\mathrm{S}-n$-Bu mit einer Intensität von $15 \%$. Das Fragment $\mathrm{Ga}-\mathrm{C} \equiv \mathrm{C}$ erzeugte einen Peak bei m/e von 92 mit einer 100\%igen Intensität. Das ${ }^{1}$ H-NMR-Spektrum zeigt erwartungsgemäß vier Multipletts bei 0.87, 1.41, 1.68 und 2.70 ppm für die Protonen der Butylfunktion und ein Singulett bei $2.47 \mathrm{ppm}$ für die Protonen der Trimethylamingruppe.

\subsubsection{Darstellung von $\mathrm{Me}_{3} \mathrm{~N} \cdot \mathrm{Ga}\left(-\mathrm{C} \equiv \mathrm{C}-\mathrm{SO}_{2}-\left(p-\mathrm{MeC}_{6} \mathrm{H}_{4}\right)\right)_{3}$}

Zur Herstellung von $\mathrm{Me}_{3} \mathrm{~N} \cdot \mathrm{Ga}\left(-\mathrm{C} \equiv \mathrm{C}-\mathrm{SO}_{2}-\left(p-\mathrm{MeC}_{6} \mathrm{H}_{4}\right)\right)_{3}$ wurde $\mathrm{GaH}_{3} \cdot \mathrm{NMe}_{3}$ mit 3 Äquivalenten $\mathrm{H}-\mathrm{C} \equiv \mathrm{C}-\mathrm{SO}_{2}-\left(p-\mathrm{MeC}_{6} \mathrm{H}_{4}\right)$ bei Raumtemperatur in Toluol umgesetzt. Nach dem Entfernen des Lösungsmittels wurde der Rückstand in Diethylether aufgenommen und filtriert. Es gelang nicht vom Produkt Einkristalle zu erhalten, da 
es sich um eine ölige Substanz handelt, die auch nicht bei tiefen Temperaturen kristallisiert. Die Analysen ergaben:

Das EI-Massenspektrum zeigt keinen Molekülionenpeak sondern nur Signale von Fragmenten und zwar bei $\mathrm{m} / \mathrm{e}$ von 202 für $\mathrm{Ga}-\mathrm{C} \equiv \mathrm{C}-\mathrm{S}-\mathrm{Ph}$ mit einer 8\%igen Intensität, bei m/e von 150 für $\mathrm{NMe}_{3} \mathrm{Ga}-\mathrm{C} \equiv \mathrm{C}$ mit einer $55 \%$ igen Intensität und bei m/e von 114 für $\mathrm{GaNMe}_{2}$ mit einer Intensität von $45 \%$. Der Peak mit einer 100\%igen Intensität wurde erzeugt durch $\mathrm{NMe}_{2} \mathrm{CH}$ bei m/e von 57 .

Das ${ }^{1}$ H-NMR-Spektrum zeigt erwartungsgemäß zwei Multipletts im aromatischen Bereich für die Protonen der Arylfunktion und ein Singulett bei $2.42 \mathrm{ppm}$ für die Protonen der Trimethylamingruppe. Weiterhin ist noch das Singulett der Protonen der p-Methylgruppe am Aromaten bei einer chemischen Verschiebung von $2.58 \mathrm{ppm} \mathrm{zu}$ erkennen.

\subsection{Austausch von $\mathrm{NMe}_{3}$ durch Arduengocarbene am $\mathrm{GaH}_{3}$}

\subsubsection{Darstellung von $\mathrm{H}_{3} \mathrm{Ga} \cdot \mathrm{C}(\mathrm{N}(\text { iso-propyl }))_{2} \mathrm{C}_{2} \mathrm{H}_{2}$}

Bei der Darstellung von $\mathrm{H}_{3} \mathrm{Ga}$-iso-propylarduengocarben wurde $\mathrm{GaH}_{3} \cdot \mathrm{NMe}_{3}$ mit einem Äquivalent des iso-Propylarduengocarbens in Toluol bei Raumtemperatur umgesetzt. Die bei der Reaktion anfallenden unlöslichen Produkte wurden abfiltriert. Nach Kristallisation in Diethylether bei $-27{ }^{\circ} \mathrm{C}$ wurde das Produkt mit einer $10 \%$ igen Ausbeute erhalten.

Die Substitution des Trimethylamins durch ein Arduengocarben ist deshalb möglich, da das Carben mit seinem freien Elektronenpaar stärker lewisbasisch ist als das Amin. 
Das Carbenaddukt ist thermisch stabiler als die Ausgangssubstanz. Es eröffnet sich hierdurch die Möglichkeit, Reaktionen bei höheren Temperaturen als $55^{\circ} \mathrm{C}$ mit aciden Verbindungen zu machen. Da das Carben selber über zwei acide Protonen verfügt, reagiert das Produkt eventuell miteinander. Dies könnte der Grund für die geringe Ausbeute und die Bildung von unlöslichen Nebenprodukten sein. Dies und eine sehr geringe Löslichkeit in den gängigen Lösungsmittel schränkt die Synthesemöglichkeiten mit diesem Produkt stark ein. Die Analysen ergaben folgendes:

Das ${ }^{1} \mathrm{H}-\mathrm{NMR}$-Spektrum zeigt deutlich ein breites Singulett bei einer chemischen Verschiebung von $4.55 \mathrm{ppm}$. Weiterhin sind mit einem Duplett bei $0.91 \mathrm{ppm}$, einem Septett bei $5.18 \mathrm{ppm}$ und einem Singulett bei $6.23 \mathrm{ppm}$ alle für dieses Arduengocarbenaddukt typischen Signale erkennbar.

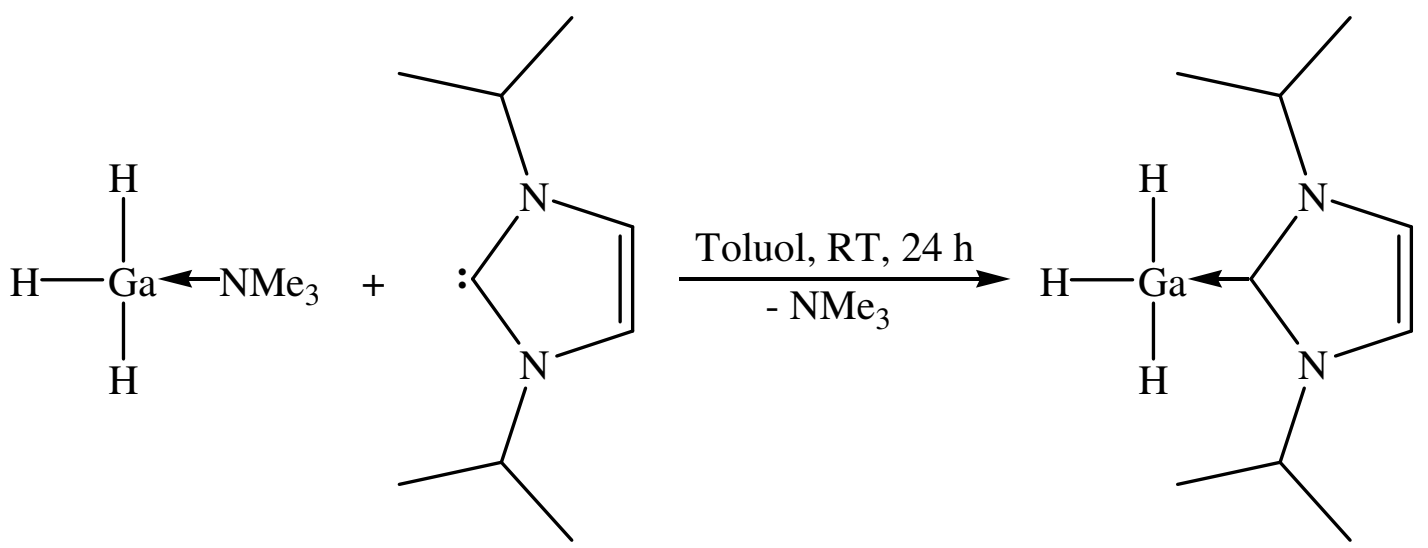

Reaktionsgleichung 3.2.1.1 


\subsubsection{Diskussion der Einkristallröntgenstrukturanalyse}

\section{von $\mathrm{H}_{3} \mathrm{Ga} \cdot \mathrm{C}(\mathrm{N}(\text { iso-propyl }))_{2} \mathrm{C}_{2} \mathrm{H}_{2}$}

Die strukturelle Untersuchung eines Einkristalls bestätigt den angenommen Aufbau des Hydrids und beweist, dass Arduengocarbene als Donor am Gallium fungieren können.

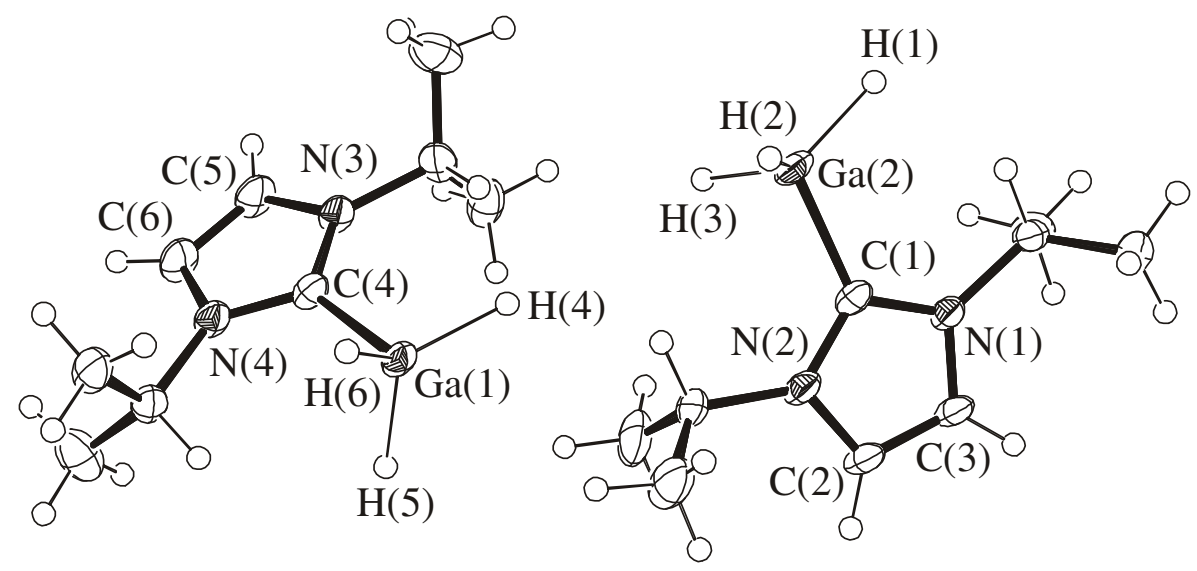

Abbildung 3.2.1.2: Struktur von $\mathrm{H}_{3} \mathrm{Ga} \cdot \mathrm{C}(\mathrm{N}(\text { iso-propyl }))_{2} \mathrm{C}_{2} \mathrm{H}_{2}$ im Kristall

Tabell 3.2.1.2: Ausgewählte Bindungslängen $[\mathrm{pm}]$ und Winkel $\left[{ }^{\circ}\right]$ von $\mathrm{H}_{3} \mathrm{Ga} \cdot \mathrm{C}(\mathrm{N}(\text { iso-propyl }))_{2} \mathrm{C}_{2} \mathrm{H}_{2}$

$\begin{array}{llll}\mathrm{Ga}(1)-\mathrm{C}(4) & 192.2(2) & \mathrm{N}(3)-\mathrm{C}(4) & 129.2(4) \\ \mathrm{N}(3)-\mathrm{C}(5) & 136.7(4) & \mathrm{C}(4)-\mathrm{N}(4) & 140.0(4) \\ \mathrm{N}(4)-\mathrm{C}(6) & 127.2(4) & \mathrm{C}(5)-\mathrm{C}(6) & 136.7(5)\end{array}$




$\begin{array}{llll}\mathrm{C}(4)-\mathrm{N}(3)-\mathrm{C}(5) & 102.8(3) & \mathrm{N}(3)-\mathrm{C}(4)-\mathrm{N}(4) & 109.9(2) \\ (\mathrm{N}(3)-\mathrm{C}(4)-\mathrm{Ga}(1) & 123.0(2) & \mathrm{N}(4)-\mathrm{C}(4)-\mathrm{Ga}(1) & 127.0(2) \\ \mathrm{C}(6)-\mathrm{N}(4)-\mathrm{C}(4) & 110.4(3) & \mathrm{C}(6)-\mathrm{C}(5)-\mathrm{N}(3) & 113.4(3) \\ \mathrm{N}(4)-\mathrm{C}(6)-\mathrm{C}(5) & 103.4(3) & & \end{array}$

Die Kristallisation erfolgte in der othorhombische Raumgruppe Pca2(1). Der Abstand Ga(1)-C(4) liegt mit 192.2 pm im Bereich einer normalen Ga-C-Einfachbindung. Das bedeutet, dass bei dieser Art Verbindung ein sehr großer stabilisierender Effekt des Donors auftritt.

\subsubsection{Darstellung von $\mathrm{H}_{3} \mathrm{Ga} \cdot \mathrm{C}(\mathrm{NMes})_{2} \mathrm{C}_{2} \mathrm{H}_{2}$}

Bei der Darstellung $\mathrm{H}_{3} \mathrm{Ga}$-iso-propylarduengocarben wurde $\mathrm{Me}_{3} \cdot \mathrm{GaH}_{3}$ mit einem Äquivalent des Mesitylarduengocarbens in Benzol bei Raumtemperatur umgesetzt. Dabei bildete sich im Laufe eines Tages die schwer lösliche Zielverbindung mit einer Ausbeute von $96 \%$. Es entstanden keine unlöslichen Polymerisationsprodukte wie beim iso-Propylderivat, was an der in kleinen Mengen vollständigen Löslichkeit in $\mathrm{CDCl}_{3}$ erkannt wurde.

Die Analysen ergaben folgendes:

Im EI-Massenspektrum ist ein Molekülionenpeak mit m/e 376 erkennbar. Durch die Isotopenverteilung und dem geringen Fragmentierungsabstand von 1 durch die Wasserstoffabspaltung, ist eine separate Intensitätangabe nicht möglich. Das größte Signal erscheint bei m/e 375 mit einer Intensität von $100 \%$. 
Im IR-Spektrum ist eine sehr starke Absorptionsbande der Ga-H-Valenzschwingung bei einer Wellenzahl von $1797 \mathrm{~cm}^{-1} \mathrm{zu}$ sehen.

Das ${ }^{1} \mathrm{H}-\mathrm{NMR}$-Spektrum zeigt eine breite Resonanz bei $3.05 \mathrm{ppm}$ für die direkt am Gallium gebundenen Protonen. Die weiteren Signale bei 2.33, 2.60, 7.39 und 7.51 ppm liegen, im für das Carben, typischen Bereich.

\subsection{Umsetzung von $\mathrm{GaH}_{3}$-Arduengocarbenadukt mit TMSI}

Ziel dieser Reaktion ist es festzustellen, ob es möglich ist, selektiv ein oder zwei Hydridwasserstoffe durch den Einsatz von Trimethylsilyiodid zu substituieren.

\subsubsection{Umsetzung von $\mathrm{H}_{3} \mathrm{Ga} \cdot \mathrm{C}(\mathrm{NMes})_{2} \mathrm{C}_{2} \mathrm{H}_{2}$ mit 1,2 und 3 Äq. TMSI}

$\mathrm{H}_{3} \mathrm{Ga} \cdot \mathrm{C}(\mathrm{NMes})_{2} \mathrm{C}_{2} \mathrm{H}_{2}$ wurde jeweis mit einem, zwei, und drei Äquivalenten TMSI in Benzol 24 h lang umgesetzt. Das entstandene TMSH wurde nach der Reaktion mit dem Lösungsmittel destillativ entfernt. Bei den Reaktionen mit ein und zwei Äquivalenten erhielt man Gemische aus Edukt und der dreifach substituierten Verbindung. Bei einer Stöchiometrie von 1:3 war die Reaktion wie erwartet nahezu quantitativ und es wurde nur $\mathrm{I}_{3} \mathrm{Ga} \cdot \mathrm{C}(\mathrm{NMes})_{2} \mathrm{C}_{2} \mathrm{H}_{2}$ isoliert, welches röntgenographisch untersucht werden konnte. Die Analysen für die dreifache Umsetzung ergaben folgendes: 
Im EI-Massenspektrum ist ein Molekülionenpeak mit m/e 753 zu erkennen. Durch die suksessive Abspaltung der Iod-Atome sind weitere Signale mit m/e 627, 499 und 373 zu sehen.

Im IR-Spektrum erscheint eine sehr starke Absoptionsbande bei einer Wellenzahl von $1733 \mathrm{~cm}^{-1}$. Die Bande der Ga-H-Valenzschwingung bei $1797 \mathrm{~cm}^{-1}$ ist nicht mehr vorhanden, was auf eine vollständige Reaktion rückschließen lässt.

Im ${ }^{1} \mathrm{H}-\mathrm{NMR}$-Spektrum fehlt das breite Hydridprotonensignal bei $3.05 \mathrm{ppm}$, das ebenfalls auf eine quantitative Umsetzung hinweist. Die vorhandenen Signale liegen alle in einem Bereich, der für den Donorsubstituenten typisch ist. Sie unterscheiden sich aber von den Resonanzen des Edukts und des freien Carbens.

\subsubsection{Diskussion der Einkristallröntgenstrukturanalyse von $\mathrm{I}_{3} \mathrm{Ga} \cdot \mathrm{C}(\mathrm{NMes})_{2} \mathrm{C}_{2} \mathrm{H}_{2}$}

Die strukturelle Untersuchung eines Einkristalls bestätigt den angenommen Aufbau des Iodids und beweist, dass alle Hydridwasserstoffe durch TMSI substituiert worden sind. Weiterhin wird gezeigt, dass Arduengocarbene bei Galliumiodid einsetzbar sind. 


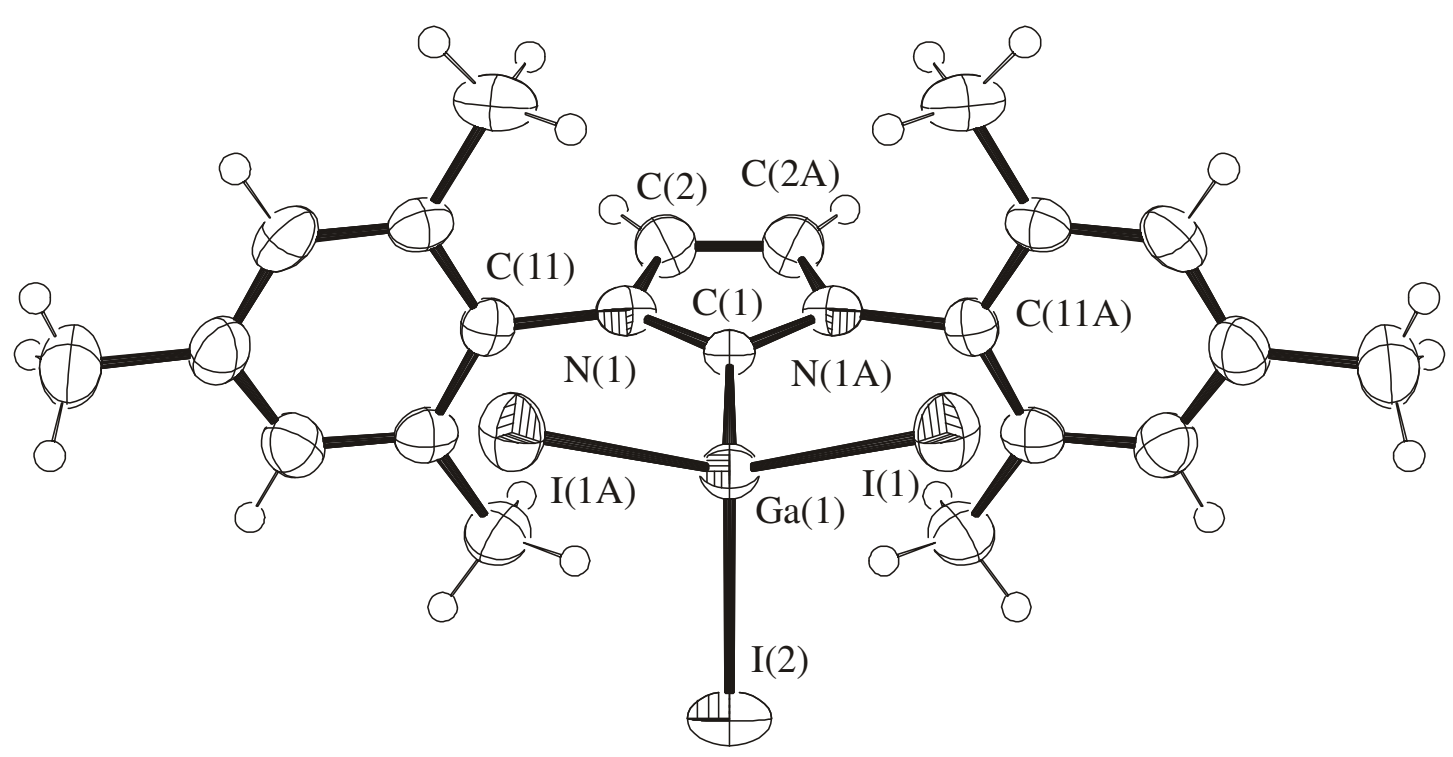

Abbildung 3.3.1.2: Struktur von $\mathrm{I}_{3} \mathrm{Ga} \cdot \mathrm{C}(\mathrm{NMes})_{2} \mathrm{C}_{2} \mathrm{H}_{2}$ im Kristall

Tabelle 3.3.1.2: Ausgewählte Bindungslängen [pm] und Winkel $\left[{ }^{\circ}\right]$ von $\mathrm{I}_{3} \mathrm{Ga} \cdot \mathrm{C}(\mathrm{NMes})_{2} \mathrm{C}_{2} \mathrm{H}_{2}$

$\begin{array}{llll}\mathrm{Ga}(1)-\mathrm{C}(1) & 204.7(9) & \mathrm{Ga}(1)-\mathrm{I}(1 \mathrm{~A}) & 253.79(10) \\ \mathrm{Ga}(1)-\mathrm{I}(1) & 253.79(10) & \mathrm{Ga}(1)-\mathrm{I}(2) & 255.15(12) \\ \mathrm{N}(1)-\mathrm{C}(1) & 133.9(8) & \mathrm{N}(1)-\mathrm{C}(2) & 136.6(9) \\ \mathrm{N}(1)-\mathrm{C}(11) & 145.6(9) & \mathrm{C}(1)-\mathrm{N}(1 \mathrm{~A}) & 133.9(8) \\ \mathrm{C}(2)-\mathrm{C}(2 \mathrm{~A}) & 134.3(15) & & \\ & & & \\ \mathrm{C}(1)-\mathrm{Ga}(1)-\mathrm{I}(1 \mathrm{~A}) & 111.77(11) & \mathrm{C}(1)-\mathrm{Ga}(1)-\mathrm{I}(1) & 111.77(11) \\ \mathrm{I}(1 \mathrm{~A})-\mathrm{Ga}(1)-\mathrm{I}(1) & 108.54(5) & \mathrm{C}(1)-\mathrm{Ga}(1)-\mathrm{I}(2) & 110.2(2) \\ \mathrm{I}(1 \mathrm{~A})-\mathrm{Ga}(1)-\mathrm{I}(2) & 107.16(3) & \mathrm{I}(1)-\mathrm{Ga}(1)-\mathrm{I}(2) & 107.16(3) \\ \mathrm{C}(1)-\mathrm{N}(1)-\mathrm{C}(2) & 110.4(6) & \mathrm{C}(1)-\mathrm{N}(1)-\mathrm{C}(11) & 128.4(6) \\ \mathrm{C}(2)-\mathrm{N}(1)-\mathrm{C}(11) & 121.2(6) & \mathrm{N}(1 \mathrm{~A})-\mathrm{C}(1)-\mathrm{N}(1) & 105.6(8)\end{array}$




$\begin{array}{lll}\mathrm{N}(1 \mathrm{~A})-\mathrm{C}(1)-\mathrm{Ga}(1) & 127.1(4) & \mathrm{N}(1)-\mathrm{C}(1)-\mathrm{Ga}(1) \\ \mathrm{C}(2 \mathrm{~A})-\mathrm{C}(2)-\mathrm{N}(1) & 106.8(4)\end{array}$

Die Kristallisation erfolgte in einer orthorhombischen Raumgruppe Pnma. Die 3 Iodatome und die Donorfunktion sind hierbei verzerrt tetraedrisch um das zentrale Galliumatom angeordnet. Die I-Ga-C-Winkel mit $111^{\circ}$ sind dabei aber nur unwesentlich größer als die I-Ga-I-Winkel mit $107^{\circ}$. Diese Winkel beweisen einen enormen sterischen Anspruch des $\mathrm{C}(\mathrm{NMes})_{2} \mathrm{C}_{2} \mathrm{H}_{2}$-Substituenten. Ein weiteres Indiz dafür ist auch die mit 205 pm sehr große Ga(1)-C(1)-Bindungslänge. Die analoge Bindung in $\mathrm{H}_{3} \mathrm{Ga} \cdot \mathrm{C}(\mathrm{N}(\text { iso-propyl }))_{2} \mathrm{C}_{2} \mathrm{H}_{2}$ ist mit $192 \mathrm{pm}$ wesentlich kürzer. Hierbei spielt natürlich auch der hohe Platzbedarf der 3 Iodatome eine Rolle, der bei $\mathrm{H}_{3} \mathrm{Ga} \cdot \mathrm{C}\left(\mathrm{N}(\right.$ iso-propyl $){ }_{2} \mathrm{C}_{2} \mathrm{H}_{2}$ mit seinen 3 hydridischen Wasserstoffen nicht so groß ist. Bei Betrachtung der Einkristallröntgenstruktur von $\mathrm{I}_{3} \mathrm{Ga} \cdot \mathrm{C}(\mathrm{NMes})_{2} \mathrm{C}_{2} \mathrm{H}_{2}$ fällt auf, dass die Mesitylfunktionen am Donor so angeordnet sind, dass sie möglicht wenig Raum in Anspruch nehmen. Ob diese Gruppen in ihrer Drehbarkeit stark gehindert sind, lässt sich auch nicht mit dem ${ }^{1} \mathrm{H}-\mathrm{NMR}-$ Spektrum beantworten, da die $o$-Methylprotonen auch dann enantiotrop sind und somit nicht unterscheidbar sind.

\subsection{Darstellung von (Dipp) ${ }_{2}$ NacNacGa-Halogeniden und Hydriden}

Die Chlor- und Iodderivate der (Dipp) ${ }_{2} \mathrm{NacNacGa-Halogenide} \mathrm{sind}$ literaturbekannt. ${ }^{[16]}$ Es war folglich wahrscheinlich, dass die Fluor- und Bromderivate synthetisierbar sein müssten. Zur Darstellung von (Dipp) ${ }_{2} \mathrm{NacNacGaBr}_{2}$ sollte eine ähnliche Strategie wie bei den Iod- und Chlorverbindungen möglich sein. Beim 
Fluorderivat soll gezeigt werden, dass eine Fluorierung mit $\mathrm{Me}_{3} \mathrm{SnF}$ nicht nur bei Übergangsmetallen ${ }^{[17,18,19,20]}$ möglich ist, sondern auch bei Galliumverbindungen.

Vielversprechend sollte auch die Synthese von (Dipp) ${ }_{2} \mathrm{NacNacGaH}_{2}$ sein, da die entsprechende Aluminiumverbindung bekannt ist. ${ }^{[21]}$

\subsubsection{Darstellung von (Dipp) ${ }_{2} \mathrm{NacNacGaBr}_{2}$}

Zur Darstellung von (Dipp) ${ }_{2} \mathrm{NacNacGaBr}_{2}$ wurde $\mathrm{GaBr}_{3}$ mit einem Äquivalent (Dipp) ${ }_{2} \mathrm{NacNacLi} \cdot \mathrm{Et}_{2} \mathrm{O}$ in Diethylether bei $-78{ }^{\circ} \mathrm{C}$ umgesetzt. Zur vollständigen Abtrennung des entstandenen LiBr musste nach dem Entfernen des Diethylethers das Produkt in $n$-Hexan aufgenommen und filtriert werden. Einkristalle wurden durch Kristallisation in Diethylether mit einer Ausbeute von $82 \%$ erhalten.
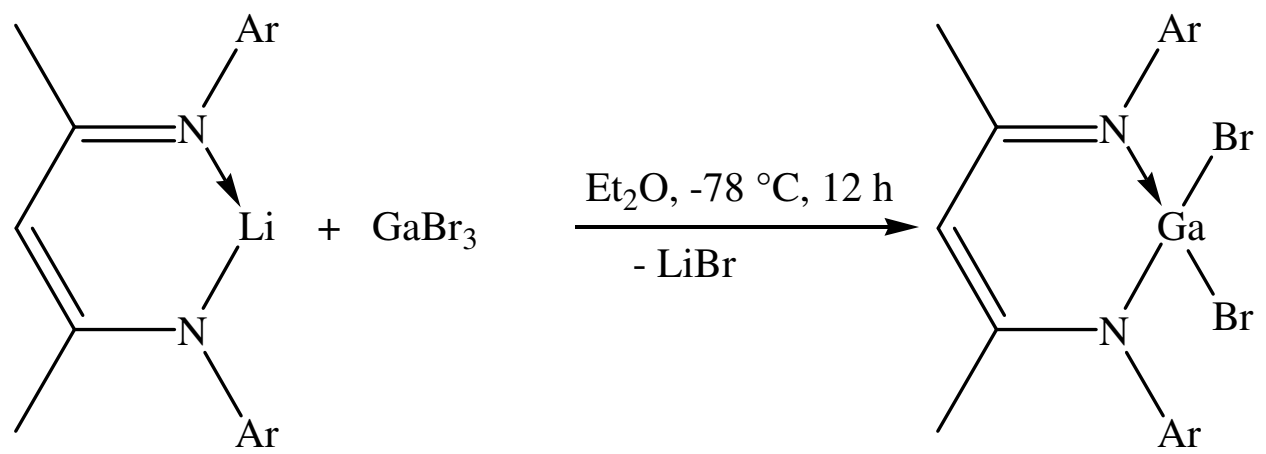

Reaktionsgleichung 3.4.1.1 
Die Analysen ergaben:

Das EI-Massenspektrum (Dipp) ${ }_{2} \mathrm{NacNacGaBr}_{2}$ zeigt einen Molekülionenpeak bei m/e 646. Bei der Fragmentierung des Moleküls wurden nacheinander beide Bromatome abgespalten, was durch Signale bei m/e 567 und 486 erkennbar ist.

Im IR-Spektrum sind zwei sehr starke Absorptionsbanden bei Wellenzahlen von 1099 und $1021 \mathrm{~cm}^{-1} \mathrm{zu}$ erkennen.

Das ${ }^{1}$ H-NMR-Spektrum zeigt ein für diese Verbindung typisches Septett bei einer chemischen Verschiebung von $3.37 \mathrm{ppm}$, das den $4 \mathrm{CH}\left(\mathrm{CH}_{3}\right)_{2}$-Protonen zugeordnet werden kann. Weiterhin sichtbar sind zwei Dubletts bei 1.20 und $1.31 \mathrm{ppm}$, welche von den 24 Methylprotonen der 4 iso-Propylgruppen stammen. Es erscheinen zwei verschiedene Signale, da die iso-Propylfunktionen in ihrer Drehbarkeit eingeschränkt sind. Das Singulett bei $1.90 \mathrm{ppm}$ ist den Protonen der am Vinamidin gebundenen Methylgruppen, das Singulett bei $5.31 \mathrm{ppm}$ dem am $\gamma$-Kohlenstoff gebundenen Proton zuzuordnen. Die Arylprotonen ergeben im Bereich von 7.20-7.30 ppm ein Multiplett.

Das $\quad{ }^{13} \mathrm{C}-\mathrm{NMR}-\mathrm{Spektrum} \quad$ von $\quad(\text { Dipp })_{2} \mathrm{NacNacGaBr}_{2}$ zeigt für das Vinamidingrundgerüst drei Resonanzen (24.15 ppm $\left(\mathrm{NC}\left(\mathrm{CH}_{3}\right)\right), 97.52 \mathrm{ppm}(\gamma-C)$, $171.18 \mathrm{ppm}(\mathrm{NC}))$. Die Dipp-Liganden liefern mehrere Resonanzen für die aromatischen Kohlenstoffkerne (124.21, 127.86, 138.19 und 144.50 ppm) und drei Signale der Kohlenstoffkerne der iso-Propylgruppen (24.57 ppm $\left(\mathrm{CH}\left(\mathrm{CH}_{3}\right)\right), 25.54$ und $28.58 \mathrm{ppm}\left(\mathrm{CH}\left(\mathrm{CH}_{3}\right)_{2}\right)$. 


\subsubsection{Diskussion der Einkristallröntgenstrukturanalyse} von (Dipp) ${ }_{2} \mathrm{NacNacGaBr}_{2}$

Die Einkristallröntgenstrukturanalyse bestätigt den angenommenen Aufbau der Zielverbindung und den zu den Chlor- und Iodderivaten analogen Reaktionsverlauf.

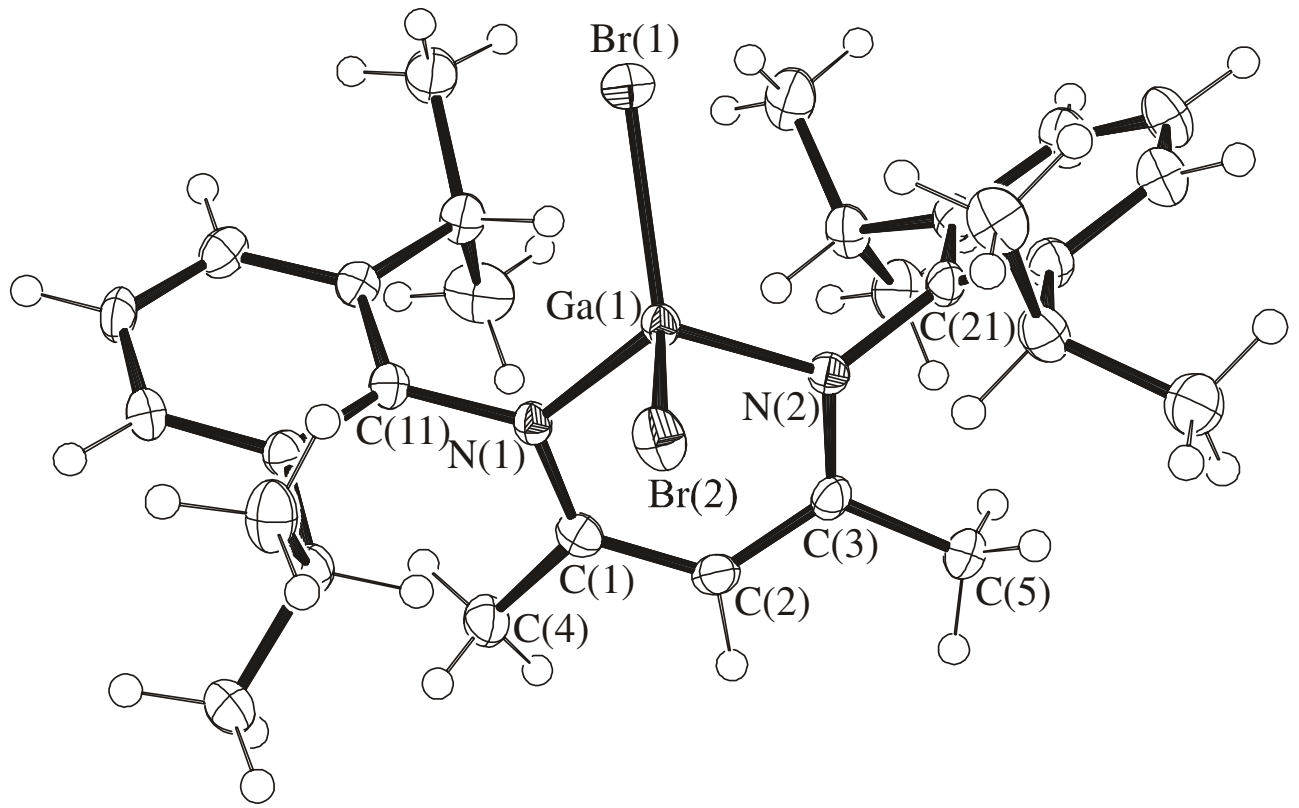

Abbildung 3.4.1.2: Struktur von (Dipp) ${ }_{2} \mathrm{NacNacGaBr}_{2}$ im Kristall

Tabelle 3.4.1.2: Ausgewählte Bindungslängen [pm] und Winkel $\left[{ }^{\circ}\right]$ von (Dipp) ${ }_{2} \mathrm{NacNacGaBr}_{2}$

$\begin{array}{llll}\mathrm{Ga}(1)-\mathrm{N}(2) & 190.8(3) & \mathrm{Ga}(1)-\mathrm{N}(1) & 192.5(3) \\ \mathrm{Ga}(1)-\mathrm{Br}(1) & 228.62(5) & \mathrm{Ga}(1)-\mathrm{Br}(2) & 232.97(5) \\ \mathrm{N}(1)-\mathrm{C}(1) & 133.5(4) & \mathrm{N}(1)-\mathrm{C}(11) & 145.9(4) \\ \mathrm{N}(2)-\mathrm{C}(3) & 134.2(4) & \mathrm{N}(2)-\mathrm{C}(21) & 145.1(4) \\ \mathrm{C}(1)-\mathrm{C}(4) & 150.1(5) & \mathrm{C}(2)-\mathrm{C}(3) & 139.7(5) \\ \mathrm{C}(3)-\mathrm{C}(5) & 150.6(4) & \end{array}$




$\begin{array}{llll}\mathrm{N}(2)-\mathrm{Ga}(1)-\mathrm{N}(1) & 99.81(11) & \mathrm{N}(2)-\mathrm{Ga}(1)-\mathrm{Br}(1) & 112.85(8) \\ \mathrm{N}(1)-\mathrm{Ga}(1)-\mathrm{Br}(1) & 116.03(8) & \mathrm{N}(2)-\mathrm{Ga}(1)-\mathrm{Br}(2) & 107.87(8) \\ \mathrm{N}(1)-\mathrm{Ga}(1)-\mathrm{Br}(2) & 107.15(8) & \mathrm{Br}(1)-\mathrm{Ga}(1)-\mathrm{Br}(2) & 112.18(19) \\ \mathrm{C}(1)-\mathrm{N}(1)-\mathrm{C}(11) & 120.1(3) & \mathrm{C}(1)-\mathrm{N}(1)-\mathrm{Ga}(1) & 116.7(2) \\ \mathrm{C}(11)-\mathrm{N}(1)-\mathrm{Ga}(1) & 122.86(19) & \mathrm{C}(3)-\mathrm{N}(2)-\mathrm{C}(21) & 119.0(3) \\ \mathrm{C}(3)-\mathrm{N}(2)-\mathrm{Ga}(1) & 116.1(2) & \mathrm{C}(21)-\mathrm{N}(2)-\mathrm{Ga}(1) & 124.9(2) \\ \mathrm{N}(1)-\mathrm{C}(1)-\mathrm{C}(2) & 123.5(3) & \mathrm{N}(1)-\mathrm{C}(1)-\mathrm{C}(4) & 120.1(3) \\ \mathrm{C}(2)-\mathrm{C}(1)-\mathrm{C}(4) & 116.4(3) & \mathrm{C}(3)-\mathrm{C}(2)-\mathrm{C}(1) & 128.7(3) \\ \mathrm{N}(2)-\mathrm{C}(3)-\mathrm{C}(2) & 123.5(3) & \mathrm{N}(2)-\mathrm{C}(3)-\mathrm{C}(5) & 118.8(3) \\ \mathrm{C}(2)-\mathrm{C}(3)-\mathrm{C}(5) & 117.7(3) & \end{array}$

Die Kristallisation erfolgte in der monoklinen Raumgruppe P2(1)/n. Der zentrale sechsgliedrige Ring ist analog zur Chlor- und Iodverbindung ${ }^{[16]}$ nicht planar sondern um die Ga(1)-C(2)-Achse gewinkelt. Durch die Winkelung wird der Abstand zwischen den Bromatomen und den 2,6-iso-Propylphenylgruppen vergrößert. Die Ursache dafür ist der sterische Anspruch der Bromatome.

\subsubsection{Darstellung von (Dipp) ${ }_{2} \mathrm{NacNacGaF}_{2}$}

Die Synthese von (Dipp) ${ }_{2} \mathrm{NacNacGaF}_{2}$ unterscheidet sich von den anderen Halogenderivaten. Die Fluorierung von Organoübergangsmetallverbindungen $^{[17,18,19,20]}$ und Hauptgruppenelementchloriden ${ }^{[22]}$ sind literaturbekannt. Zur Darstellung wurde (Dipp) ${ }_{2} \mathrm{NacNacGaI}_{2}$ mit zwei Äquivalenten $\mathrm{Me}_{3} \mathrm{SnF}$ in Benzol 
bei Raumtemperatur umgesetzt. Da sich das Fluorierungsreagenz sehr schlecht in Benzol löst, betrug die Reaktionszeit 4 Wochen. Eine Erhöhung der Temperatur hatte keinen Einfluß auf die Reaktionsgeschwindigkeit, sondern führte zu einer Zersetzung der Reaktionskomponenten. Dies äußerte sich durch eine zunehmende Braunfärbung der Reaktionslösung. Das Ende der Reaktion wurde durch den Verbrauch des schlecht löslichen $\mathrm{Me}_{3} \mathrm{SnF}$ angezeigt. Durch Kristallisation in Diethylether wurden für die röntgenographische Untersuchung geeignete Einkristalle erhalten.

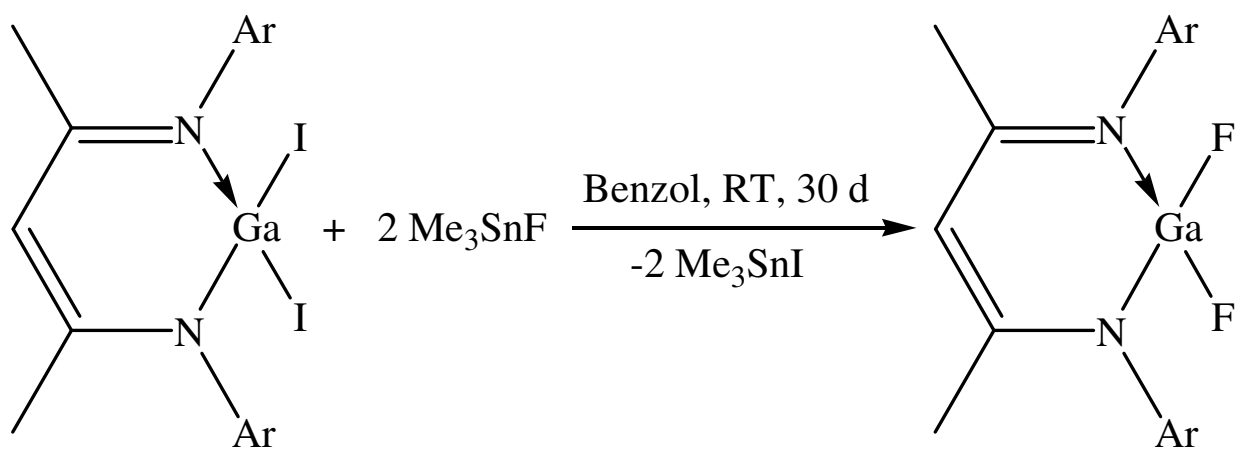

\section{Reaktionsgleichung 3.4.2.1}

Die Analysen ergaben:

Das EI-Massenspektrum (Dipp) ${ }_{2} \mathrm{NacNacGaF}_{2}$ zeigt einen Molekülionenpeak bei m/e 524. Bei der Fragmentierung des Moleküls wurden nacheinander beide Fluoratome abgespalten, was durch Signale bei m/e 505 und 486 erkennbar ist.

Das ${ }^{1}$ H-NMR-Spektrum zeigt ein für diese Verbindung typisches Septett bei einer chemischen Verschiebung von $3.30 \mathrm{ppm}$, das den $4 \mathrm{CH}\left(\mathrm{CH}_{3}\right)_{2}$-Protonen zugeordnet werden kann. Weiterhin sichtbar sind zwei Dubletts bei 1.09 und 1.44 ppm, welche von den 24 Methylprotonen der 4 iso-Propylgruppen stammen. Das Singulett bei $1.52 \mathrm{ppm}$ ist den Protonen der am Vinamidin gebundenen Methylgruppen, das 
Singulett bei $4.87 \mathrm{ppm}$ dem am $\gamma$ Kohlenstoff gebundenen Proton zuzuordnen. Die Arylprotonen ergeben im Bereich von 7.05 - 7.13 ppm ein Multiplett.

Das $\quad{ }^{13} \mathrm{C}-\mathrm{NMR}-\mathrm{Spektrum}$ von $\quad(\mathrm{Dipp})_{2} \mathrm{NacNacGaF}_{2}$ zeigt für das Vinamidingrundgerüst drei Signale $\left(20.74 \mathrm{ppm} \quad\left(\mathrm{NC}\left(\mathrm{CH}_{3}\right)\right), 95.75 \mathrm{ppm} \quad(\gamma-C)\right.$, 172.36 ppm (NC)). Die Dipp-Liganden liefern mehrere Resonanzen für die aromatischen Kohlenstoffkerne (138.02, 141.24, 142.76 und 144.38 ppm) und drei Signale der Kohlenstoffkerne der iso-Propylgruppen (23.39 ppm $\left(\mathrm{CH}\left(\mathrm{CH}_{3}\right)\right), 24.60$ und $28.60 \mathrm{ppm}\left(\mathrm{CH}\left(\mathrm{CH}_{3}\right)_{2}\right)$.

Das ${ }^{19}$ F-NMR-Spektrum von (Dipp) ${ }_{2} \mathrm{NacNacGaF}_{2}$ zeigt ein Singulett bei einer chemischen Verschiebung von 38.34 ppm, welches den beiden Fluoratomen zuzuordnen ist.

\subsubsection{Diskussion der Einkristallröntgenstrukturanalyse von (Dipp) ${ }_{2} \mathrm{NacNacGaF}_{2}$}

Die Einkristallröntgenstrukturanalyse bestätigt den angenommenen Aufbau der Zielverbindung und beweist ebenfalls den angenommenen Reaktionsverlauf. 


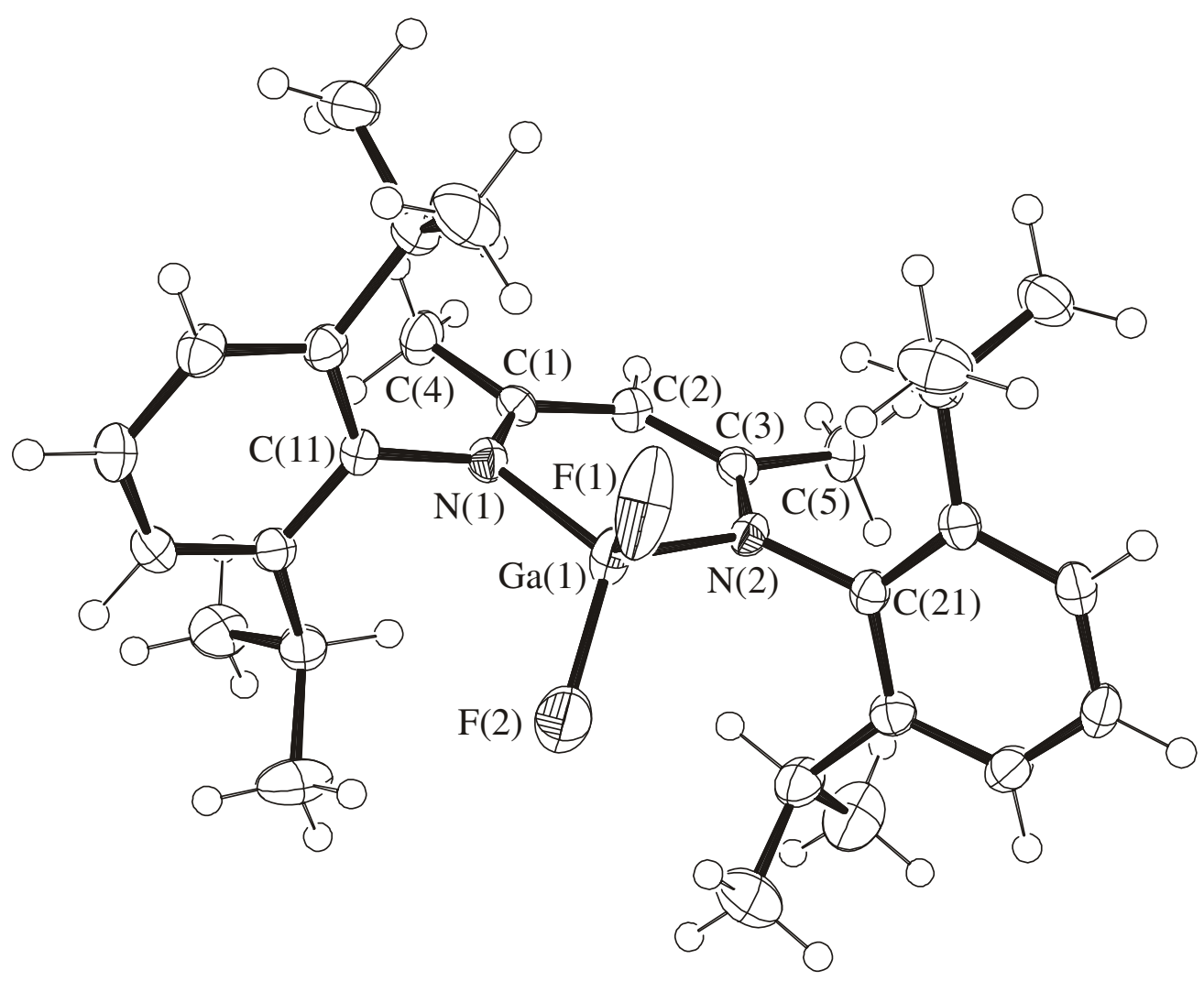

Abbildung 3.4.2.2: Struktur von (Dipp) ${ }_{2} \mathrm{NacNacGaF}_{2}$ im Kristall

Tabelle 3.4.2.2: Ausgewählte Bindungslängen $[\mathrm{pm}]$ und -winkel $\left[{ }^{\circ}\right]$ von (Dipp) ${ }_{2} \mathrm{NacNacGaF}_{2}$.

$\begin{array}{llll}\mathrm{Ga}(1)-\mathrm{F}(2) & 175.55(17) & \mathrm{Ga}(1)-\mathrm{F}(1) & 177.30(17) \\ \mathrm{Ga}(1)-\mathrm{N}(1) & 189.55(19) & \mathrm{Ga}(1)-\mathrm{N}(2) & 189.93(19) \\ \mathrm{N}(1)-\mathrm{C}(1) & 134.6(3) & \mathrm{N}(1)-\mathrm{C}(11) & 145.4(3) \\ \mathrm{N}(2)-\mathrm{C}(3) & 134.0(3) & \mathrm{N}(2)-\mathrm{C}(21) & 145.6(3) \\ \mathrm{C}(1)-\mathrm{C}(2) & 139.5(3) & \mathrm{C}(1)-\mathrm{C}(4) & 150.2(3) \\ \mathrm{C}(2)-\mathrm{C}(3) & 139.9(3) & \mathrm{C}(3)-\mathrm{C}(5) & 149.6(3)\end{array}$




$\begin{array}{llll}\mathrm{F}(2)-\mathrm{Ga}(1)-\mathrm{F}(1) & 103.70(10) & \mathrm{F}(2)-\mathrm{Ga}(1)-\mathrm{N}(1) & 111.89(9) \\ \mathrm{F}(1)-\mathrm{Ga}(1)-\mathrm{N}(1) & 114.02(9) & \mathrm{F}(2)-\mathrm{Ga}(1)-\mathrm{N}(2) & 114.46(9) \\ \mathrm{F}(1)-\mathrm{Ga}(1)-\mathrm{N}(2) & 112.41(8) & \mathrm{N}(1)-\mathrm{Ga}(1)-\mathrm{N}(2) & 100.79(8) \\ \mathrm{C}(1)-\mathrm{N}(1)-\mathrm{C}(11) & 119.97(18) & \mathrm{C}(1)-\mathrm{N}(1)-\mathrm{Ga}(1) & 121.23(15) \\ \mathrm{C}(11)-\mathrm{N}(1)-\mathrm{Ga}(1) & 118.78(14) & \mathrm{C}(3)-\mathrm{N}(2)-\mathrm{C}(21) & 121.83(19) \\ \mathrm{C}(3)-\mathrm{N}(2)-\mathrm{Ga}(1) & 120.95(16) & \mathrm{C}(21)-\mathrm{N}(2)-\mathrm{Ga}(1) & 117.21(14) \\ \mathrm{N}(1)-\mathrm{C}(1)-\mathrm{C}(2) & 123.6(2) & \mathrm{N}(1)-\mathrm{C}(1)-\mathrm{C}(4) & 118.7(2) \\ \mathrm{C}(2)-\mathrm{C}(1)-\mathrm{C}(4) & 117.7(2) & \mathrm{C}(1)-\mathrm{C}(2)-\mathrm{C}(3) & 129.4(2) \\ \mathrm{N}(2)-\mathrm{C}(3)-\mathrm{C}(2) & 124.0(2) & \mathrm{N}(2)-\mathrm{C}(3)-\mathrm{C}(5) & 118.8(2) \\ \mathrm{C}(2)-\mathrm{C}(3)-\mathrm{C}(5) & 117.2(2) & \end{array}$

Die Kristallisation erfolgte analog zum Bromderivat ebenfalls in der monoklinen Raumgruppe P2(1)/n. Im Gegensatz zum Bromderivat ist der zentrale sechsgliedrige Ring planar. Das zeigt, dass die Fluoratome genügend Platz haben und es nicht notwendig ist die Dipp-Funktion wegzudrücken und damit den Vinamidinring zu deformieren. Der N-Ga-N-Winkel ist mit $100^{\circ}$ nur ein Grad größer und die Ga-N-Bindungslänge ist mit 190 pm zwei Pikometer kürzer als bei der analogen Bromverbindung. Die aromatischen Funktionen stehen senkrecht zum Vinamidingrundgerüst um den Fluoratomen am besten auszuweichen. 


\subsubsection{Darstellung von (Dipp) ${ }_{2} \mathrm{NacNacGaH}_{2}$ aus (Dipp) ${ }_{2} \mathrm{NacNacGaI}_{2}$ oder (Dipp) ${ }_{2} \mathrm{NacNacGaCl}_{2}$ und Superhydrid}

Die Darstellung von (Dipp) ${ }_{2} \mathrm{NacNacGaH}_{2}$ erfolgte durch Umsetzung von (Dipp) ${ }_{2} \mathrm{NacNacGaCl}_{2}$ oder (Dipp) ${ }_{2} \mathrm{NacNacGaI}_{2}$ mit 2 Äquivalenten $\mathrm{LiHBEt}_{3}$ (Superhydrid). Dazu wurde das (Dipp) ${ }_{2} \mathrm{NacNacGaHal}_{2}$ in Benzol gelöst und eine $1 \mathrm{M}$ THF-Lösung des Superhydrids zugetropft. Innerhalb von $24 \mathrm{~h}$ bildete sich bei Raumtemperatur die Zielverbindung und LiHal. LiI ist im Benzol/THF-Gemisch vollständig löslich. Es wurde entfernt, indem das Lösungsmittelgemisch abdestilliert, und der feste Rückstand in $n$-Hexan aufgenommen wurde. Dabei löste sich die Zielverbindung, wobei das LiI als Feststoff abfiltriert werden konnte. Der Nachteil dieser Methode ist die signifikante Löslichkeit von LiI in $n$-Hexan. Die Elementaranalyse zeigt eine 6\%ige Verunreinigung mit I. Die Verunreinigung mit dem Nebenprodukt kann vermieden werden, wenn vom (Dipp) ${ }_{2} \mathrm{NacNacGaCl}_{2}$ ausgegangen wird. LiCl lag bei der Darstellung im Benzol/THF-Gemisch schon weitestgehend als Feststoff vor. Bei der Aufarbeitung mit $n$-Hexan wurde es vollständig durch Filtration entfernt. Die Gallium-Chlor-Bindung ist nicht zu stark, um sich der Hydrierung durch das Superhydrid zu entziehen. Somit ist es nicht nötig von der Iodverbindung mit der schwächeren Gallium-Iod-Bindung auszugehen. Das Superhydrid hat den Vorteil, dass es in einem Benzol/THF-Gemisch gelöst ist und dadurch eine ausreichende Reaktivität vorliegt. 


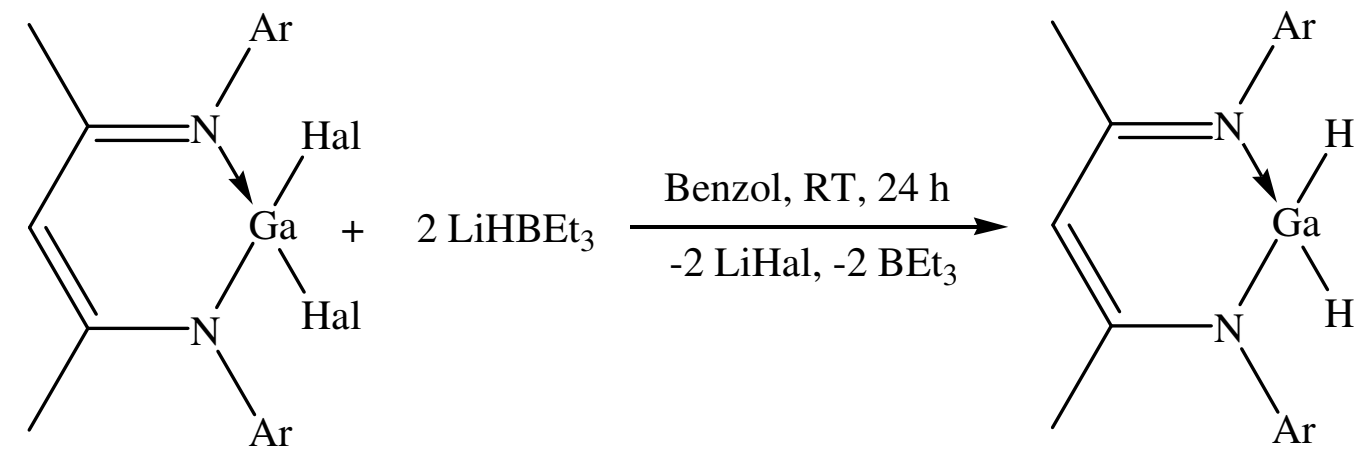

Reaktionsgleichung 3.4.3.1.1

Andere Hydrierungsmittel wie z.B. NaH, KH oder LiH lösen sich nicht, so das keine Reaktion eintritt.
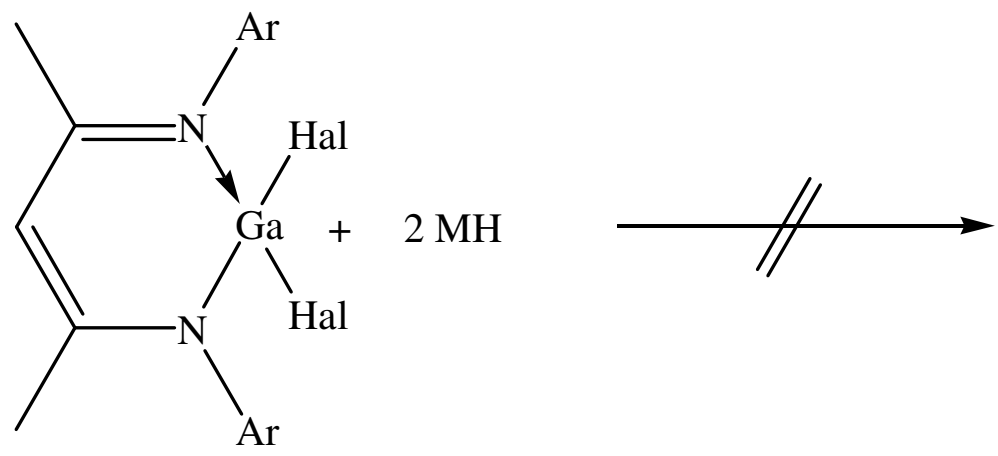

Reaktionsgleichung 3.4.3.1.2

Das lösliche Hydrierungsmittel $\mathrm{LiAlH}_{4}$ neigt zum Austausch des Zentralatoms der Ausgangsverbindung. Dies wurde bei Versuchen von P.P.Power und seinen Mitarbeitern gezeigt, als er (Dipp) ${ }_{2} \mathrm{NacNacBiH}_{2}$ aus (Dipp) ${ }_{2} \mathrm{NacNacBiHal}_{2}$ und $\mathrm{LiAlH}_{4}$ herstellen wollte und (Dipp) ${ }_{2} \mathrm{NacNacAlH}_{2}$ erhielt. ${ }^{[21]} \mathrm{LiGaH}_{4}$ ist als Hydrierungsmittel ungeeignet, da es wegen seiner Zersetzlichkeit als Feststoff schlecht zu isolieren ist und somit nicht stöchiometrisch eingesetzt werden kann. 
(Dipp) ${ }_{2} \mathrm{NacNacAlH}_{2}$ wird vorzugsweise aus $\mathrm{AlH}_{3} \cdot \mathrm{NMe}_{3}$ und (Dipp) ${ }_{2} \mathrm{NacNacH}$ hergestellt. ${ }^{[23]}$ Eine analoge Umsetzung mit $\mathrm{GaH}_{3} \cdot \mathrm{NMe}_{3}$ funktioniert nicht. Die hydridischen Wasserstoffatome sind beim $\mathrm{GaH}_{3} \cdot \mathrm{NMe}_{3}$ viel weniger reaktiv als beim Aluminiumhydrid, so dass das schwach acide Proton des (Dipp) ${ }_{2} \mathrm{NacNac-Restes} \mathrm{nicht}$ mit den hydridischen Wasserstoffen $\mathrm{zu} \mathrm{H}_{2}$ reagiert. Es ist auch nicht möglich die Reaktion durch stärkeres Erhitzen voranzutreiben, da sich $\mathrm{GaH}_{3} \cdot \mathrm{NMe}_{3}$ bei mehr als $55{ }^{\circ} \mathrm{C}$ schneller zersetzt, als es reagiert.

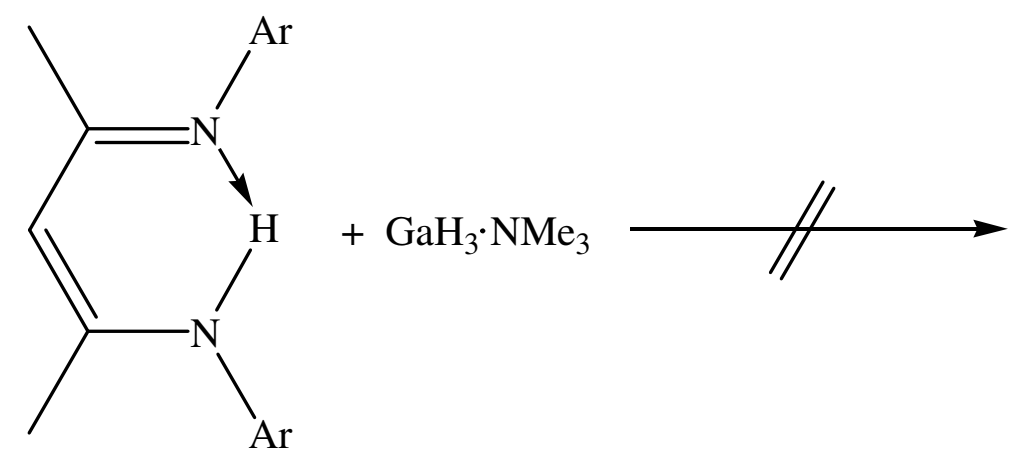

Reaktionsgleichung 3.4.3.1.3 
Die Analysen ergaben:

Das EI-Massenspektrum (Dipp) ${ }_{2} \mathrm{NacNacGaH}_{2}$ zeigt einen Molekülionenpeak bei m/e 488. Bei der Fragmentierung des Moleküls wurden nacheinander beide Hydridwasserstoffe abgespalten, was durch Signale bei m/e 487 und 486 erkennbar ist. Da die Massen der Fragmente sich nur durch ein Wasserstoffatom unterscheiden ist eine Angabe der Intensität fragwürdig, da sich die Signale durch die Isotopenverteilung überlagern. Der größte Molekülionenpeak ist sichtbar bei m/e 487 . Zur Intensität von $100 \%$ tragen alle drei Komponenten bei (M, M-H, M-2H).

Das IR-Spektrum zeigt zwei starke Absorptionsbanden die im Bereich der Ga-H-Valenzschwingung liegen bei Wellenzahlen von 1893 und $1861 \mathrm{~cm}^{-1}$.

Das ${ }^{1} \mathrm{H}-\mathrm{NMR}$-Spektrum zeigt ein für diese Verbindung typisches Septett bei einer chemischen Verschiebung von $3.20 \mathrm{ppm}$, das den $4 \mathrm{CH}\left(\mathrm{CH}_{3}\right)_{2}$-Protonen zugeordnet werden kann. Weiterhin sichtbar sind zwei Dubletts bei 1.19 und 1.23 ppm, welche von den 24 Methylprotonen der 4 iso-Propylgruppen stammen. Das Singulett bei $1.52 \mathrm{ppm}$ ist den Protonen der am Vinamidin gebundenen Methylgruppen, das Singulett bei $4.85 \mathrm{ppm}$ dem am $\gamma$-Kohlenstoff gebundenen Proton zuzuordnen. Die Arylprotonen ergeben im Bereich von 7.10 - 7.24 ppm ein Multiplett. Ein sehr breite Resonanz bei 4.58 ppm ist zweifelsfrei den am Gallium gebundenen hydridischen Protonen zuzuordnen.

Das $\quad{ }^{13} \mathrm{C}-\mathrm{NMR}-\mathrm{Spektrum}$ von $\quad$ (Dipp) ${ }_{2} \mathrm{NacNacGaH}_{2}$ zeigt für das Vinamidingrundgerüst drei Signale $\left(22.93 \mathrm{ppm}\left(\mathrm{NC}\left(\mathrm{CH}_{3}\right)\right), 94.43 \mathrm{ppm} \quad(\gamma-C)\right.$, 168.13 ppm (NC)). Die Dipp-Liganden liefern mehrere Resonanzen für die aromatischen Kohlenstoffkerne (124.64, 127.14, 1141.09 und 144.11 ppm) und drei 
Signale der Kohlenstoffkerne der iso-Propylgruppen (24.46 ppm $\left(\mathrm{CH}\left(\mathrm{CH}_{3}\right)\right), 25.52$ und $28.30 \mathrm{ppm}\left(\mathrm{CH}\left(\mathrm{CH}_{3}\right)_{2}\right)$.

\subsubsection{Diskussion der Einkristallröntgenstrukturanalyse von (Dipp) ${ }_{2} \mathrm{NacNacGaH}_{2}$}

Die Einkristallröntgenstrukturanalyse bestätigt den angenommenen Aufbau der Zielverbindung und beweist ebenfalls den angenommenen Reaktionsverlauf.

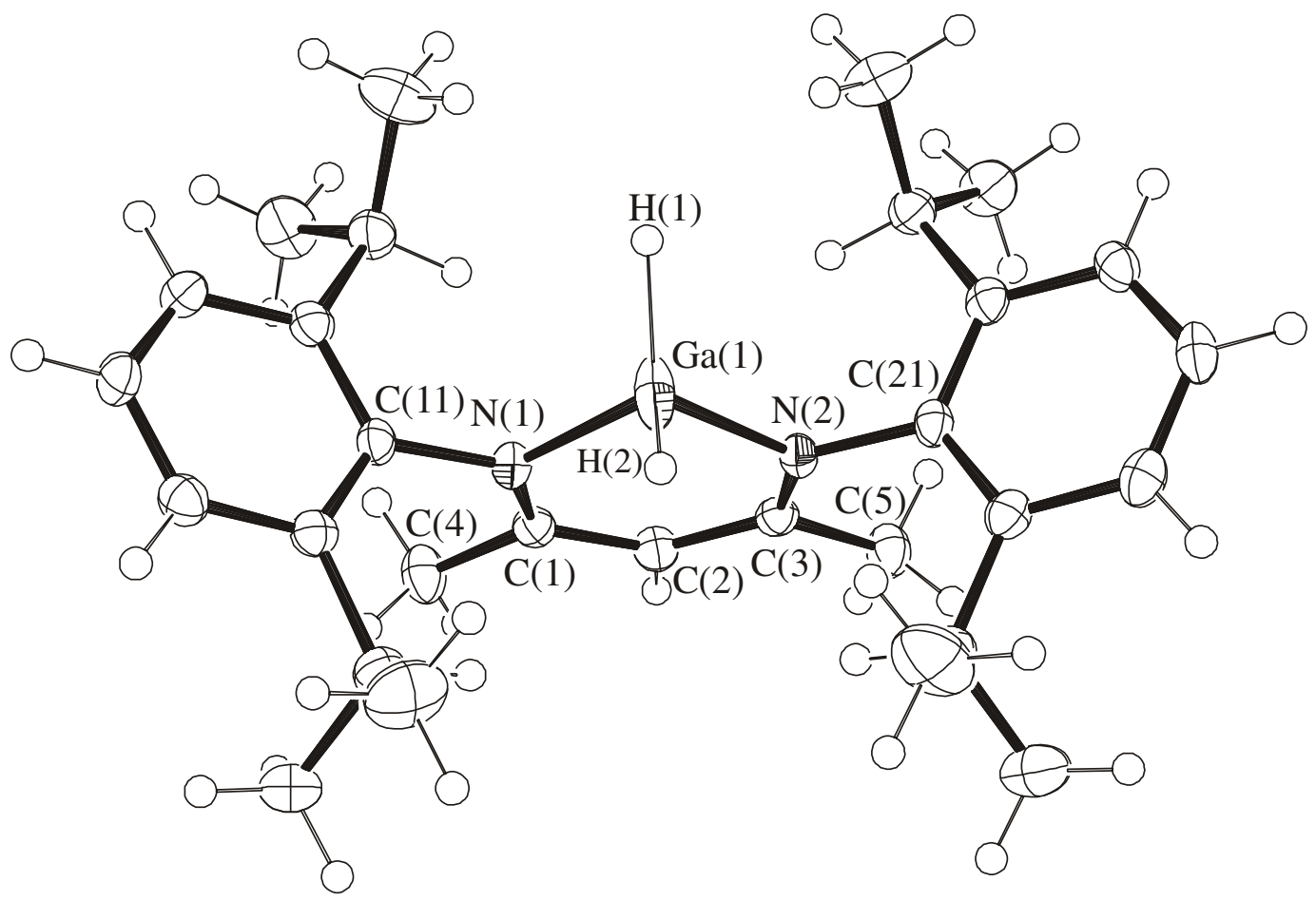

Abbildung 3.4.3.2: Struktur von (Dipp) ${ }_{2} \mathrm{NacNacGaH}_{2}$ im Kristall 
Tabelle 3.4.3.2: Ausgewählte Bindungslängen $[\mathrm{pm}]$ und -winkel $\left[{ }^{\circ}\right]$ von (Dipp) ${ }_{2} \mathrm{NacNacGaH}_{2}$.

$\begin{array}{llll}\mathrm{Ga}(1)-\mathrm{N}(1) & 195.95(14) & \mathrm{Ga}(1)-\mathrm{N}(2) & 196.95(14) \\ \mathrm{N}(1)-\mathrm{C}(1) & 132.9(2) & \mathrm{N}(1)-\mathrm{C}(11) & 144.9(2) \\ \mathrm{N}(2)-\mathrm{C}(3) & 132.6(2) & \mathrm{N}(2)-\mathrm{C}(21) & 144.6(2) \\ \mathrm{C}(1)-\mathrm{C}(2) & 140.2(2) & \mathrm{C}(1)-\mathrm{C}(4) & 150.3(2) \\ \mathrm{C}(2)-\mathrm{C}(3) & 140.2(2) & \mathrm{C}(3)-\mathrm{C}(5) & 150.7(2) \\ \mathrm{N}(1)-\mathrm{Ga}(1)-\mathrm{N}(2) & 95.66(6) & \mathrm{C}(1)-\mathrm{N}(1)-\mathrm{C}(11) & 121.78(14) \\ \mathrm{C}(1)-\mathrm{N}(1)-\mathrm{Ga}(1) & 123.87(11) & \mathrm{C}(11)-\mathrm{N}(1)-\mathrm{Ga}(1) & 114.35(11) \\ \mathrm{C}(3)-\mathrm{N}(2)-\mathrm{C}(21) & 120.51(14) & \mathrm{C}(3)-\mathrm{N}(2)-\mathrm{Ga}(1) & 124.07(11) \\ \mathrm{C}(21)-\mathrm{N}(2)-\mathrm{Ga}(1) & 115.38(11) & \mathrm{N}(1)-\mathrm{C}(1)-\mathrm{C}(2) & 123.84(15) \\ \mathrm{N}(1)-\mathrm{C}(1)-\mathrm{C}(4) & 119.26(15) & \mathrm{C}(2)-\mathrm{C}(1)-\mathrm{C}(4) & 116.90(15) \\ \mathrm{C}(3)-\mathrm{C}(2)-\mathrm{C}(1) & 128.84(16) & \mathrm{N}(2)-\mathrm{C}(3)-\mathrm{C}(2) & 123.67(15) \\ \mathrm{N}(2)-\mathrm{C}(3)-\mathrm{C}(5) & 118.98(15) & \mathrm{C}(2)-\mathrm{C}(3)-\mathrm{C}(5) & 117.34(15)\end{array}$

Die Kristallisation erfolgte analog zu den Brom- und Fluorderivaten ebenfalls in der monoklinen Raumgruppe P2(1)/n. Im Gegensatz zum Bromderivat und analog zur Fluorverbindung ist der zentrale sechsgliedrige Ring planar. Das zeigt, dass die Wasserstoffatome obwohl sie hydridisch und somit etwas vergrößert sind genügend Platz haben und es nicht notwendig ist die Dipp-Funktion wegzudrücken und damit den Vinamidinring zu deformieren. Der N-Ga-N-Winkel ist beim Hydrid mit $96^{\circ}$ vier Grad kleiner als beim Fluorid. Die Ga-N-Bindungslänge ist mit 196 pm sechs Pikometer länger als bei der analogen Fluorverbindung. Das lässt den Schluss zu, dass die hydridischen Wasserstoffe sterisch anspruchsvoller und somit größer als die 
Fluoratome sind. Die Ga-N-Bindungen und der N-Ga-N-Winkel der analogen Ga(I)-Verbindung ${ }^{[24]} \quad$ (N-Ga-N-Winkel $87.5^{\circ}, \quad$ N-Ga-Bindungen 205 pm) sind unterschiedlich zum Hydrid. Die aromatischen Funktionen stehen senkrecht zum Vinamidingrundgerüst, um den Wasserstoffatomen am besten auszuweichen.

\subsubsection{Darstellung von (Dipp) ${ }_{2}$ NacNacGaHCl}

Zur Darstellung von (Dipp) ${ }_{2} \mathrm{NacNacGaHCl}$ wurde (Dipp) ${ }_{2} \mathrm{NacNacGaH}_{2}$ mit einem Äquivalent TMSCl in Benzol umgesetzt. Das nach dem Entfernen aller flüchtigen Bestandteile erhaltene Produkt wurde ${ }^{1}$ H-NMR-spektroskopisch und massenspektrometrisch untersucht. Das ${ }^{1} \mathrm{H}-\mathrm{NMR}-\mathrm{Spektrum}$ zeigt Signale von drei Verbindungen vom Typ (Dipp) ${ }_{2} \mathrm{NacNacGaR}_{2}$ die im Verhältnis 1:1:1 auftreten. Jeweils ein Satz von Signalen kann (Dipp) ${ }_{2} \mathrm{NacNacGaCl}_{2}{ }^{[16]}$ und (Dipp) ${ }_{2} \mathrm{NacNacGaH}_{2}$ zugeordnet werden. Der dritte Satz beschreibt eine neue Verbindung. Signale vom Liganden sind nicht vorhanden. Das lässt den Schluss zu, dass sich ein Gleichgewicht zwischen Edukt, der zweifach chlorierten Verbindung und der Zielverbindung einstellt. Das EI-Massenspektrum weist nur auf die Existenz des Zielproduktes hin. Ein Molekülionenpeak von (Dipp) ${ }_{2} \mathrm{NacNacGaCl}_{2}$ ist nicht erkennbar. Möglicherweise wird das Zielprodukt bei milderen Bedingungen (niedrigere Sublimationstemperatur) detektiert als das Dichlorid. Die Analysen ergaben:

Im EI-Massenspektrum ist ein Molekülionenpeak von (Dipp) ${ }_{2} \mathrm{NacNacGaHCl}$ bei m/e $523 \mathrm{zu}$ erkennen. Fragmente sind nicht zuzuordnen, da sie auch vom Edukt stammen können, das laut ${ }^{1} \mathrm{H}-\mathrm{NMR}-$ Spektrum ebenfalls vorhanden war. 
Das ${ }^{1} \mathrm{H}$-NMR-Spektrum (bezieht sich nur auf (Dipp) ${ }_{2} \mathrm{NacNacGaHCl)}$ zeigt ein für diese Verbindung typisches Septett bei einer chemischen Verschiebung von 3.62 ppm, das den $4 \mathrm{CH}\left(\mathrm{CH}_{3}\right)_{2}$-Protonen zugeordnet werden kann. Weiterhin sichtbar sind zwei Dubletts bei 1.08 und 1.15 ppm, welche von den 24 Methylprotonen der 4 iso-Propylgruppen stammen. Das Singulett bei $1.53 \mathrm{ppm}$ ist den Protonen der am Vinamidin gebundenen Methylgruppen, das Singulett bei $4.79 \mathrm{ppm}$ dem am $\gamma$ Kohlenstoff gebundenen Proton zuzuordnen. Die Arylprotonen ergeben im Bereich von $6.90-7.20 \mathrm{ppm}$ ein Multiplett. Ein sehr breites Signal bei $5.45 \mathrm{ppm}$ ist zweifelsfrei dem am Gallium gebundenen hydridischen Proton zuzuordnen.

\subsection{Untersuchung der Reaktivität von (Dipp) ${ }_{2} \mathrm{NacNacGaH}_{2}$ gegenüber unterschiedlichen Funktionalitäten}

\subsubsection{Umsetzung von (Dipp) ${ }_{2} \mathrm{NacNacGaH}_{2}$ mit $\mathrm{H}-\mathrm{C} \equiv \mathrm{C}-\mathrm{Ph}$}

Nachdem gezeigt wurde, dass Phenylacetylen mit Galliumhydridtrimethylamin bei $55^{\circ} \mathrm{C}$ erfolgreich reagiert, lag es nahe, die analoge Reaktion mit (Dipp) ${ }_{2} \mathrm{NacNacGaH}_{2}$ durchzuführen. Bei Temperaturen von $55^{\circ} \mathrm{C}$ und $110{ }^{\circ} \mathrm{C}$ fand weder eine Reaktion noch Zersetzung statt. Da das verwendete Galliumhydrid thermisch stabil ist, wurde der Reaktionsversuch in siedendem $p$-Xylol bei $141^{\circ} \mathrm{C}$ gemacht. Bei einer anfänglichen Reaktionszeit von $12 \mathrm{~h}$ wurde weder eine Produktbildung noch eine signifikante Zersetzung des Hydrids ${ }^{1} \mathrm{H}-\mathrm{NMR}$-spektroskopisch nachgewiesen. Die Reaktionszeit wurde daraufhin schrittweise bis auf 7 Tage ausgedehnt, worauf nur noch (Dipp) ${ }_{2} \mathrm{NacNacH}^{[25]}$, also das Zersetzungsprodukt vorhanden war. Das lässt die 
Erkenntnis zu, dass (Dipp) ${ }_{2} \mathrm{NacNacGaH}_{2}$ wesentlich unreaktiver ist als Galliumhydridaddukte.

\subsubsection{Umsetzung von (Dipp) ${ }_{2} \mathrm{NacNacGaH}_{2}$ mit 1,2-cis-Diphenylethen}

Ziel dieser Reaktion war es einen dreigliedrigen Ga-C-C-Ring durch Eliminierung von $\mathrm{H}_{2}$ zu bilden. Dazu wurde (Dipp) ${ }_{2} \mathrm{NacNacGaH}_{2}$ mit 9 Äquivalenten 1,2-cisDiphenylethen in siedendem Benzol $24 \mathrm{~h}$ lang gerührt. Nach dem Entfernen aller flüchtigen Bestandteile konnte durch ein ${ }^{1} \mathrm{H}-\mathrm{NMR}$-Spektrum nur Edukte festgestellt werden.

\subsubsection{Umsetzung von (Dipp) ${ }_{2} \mathrm{NacNacGaH}_{2}$ mit ICN}

Ziel dieser Reaktion war es, analog zur Aluminiumverbindung ein Pseudohalogenderivat darzustellen. Die ${ }^{1}$ H-NMR-spektroskopische Untersuchung ergab, dass ausschließlich (Dipp) ${ }_{2} \mathrm{NacNacGaI}_{2}$ erhalten wurde, was literaturbekannt ist. $^{[16]}$ Die Tendenz mit einem Halogen eine Bindung einzugehen, ist demnach viel größer als eine Ga-C-Bindung zu bilden. Der Versuch HI mit einem Arduengocarben abzufangen, führte zum gleichen Resultat.

\subsubsection{Umsetzung von (Dipp) ${ }_{2} \mathrm{NacNacGaH}_{2}$ mit Benzonitril}

Aluminiumhydride bilden mit Nitrilen bei höheren Temperaturen interessante Cluster. ${ }^{[10]}$ Galiumtrihydridaddukte sind thermisch nicht ausreichend belastbar, um 
solche Reaktionen einzugehen. Mit (Dipp) ${ }_{2} \mathrm{NacNacGaH}_{2}$ wurde ein bei hohen Temperaturen beständiger Vertreter dieser Verbindungsklasse eingesetzt. In mehreren Versuchen wurde die Reaktionstemperatur durch die Wahl von hochsiedenden Lösungsmittel, wie Toluol $\left(110^{\circ} \mathrm{C}\right)$ und Xylol $\left(141^{\circ} \mathrm{C}\right)$ gesteigert. Es trat aber keine Reaktion ein, was ${ }^{1}$ H-NMR-spektroskopische Befunde zeigten. Als letzte Steigerung wurde Benzonitril als Lösungsmittel und Reagenz eingesetzt. Bei diesen drastischen Bedingungen $\left(180^{\circ} \mathrm{C}\right)$ zersetzte sich das Galliumhydrid allerdings, und es wurde ${ }^{1} \mathrm{H}-\mathrm{NMR}$-spektroskopisch (Dipp) ${ }_{2} \mathrm{NacNacH}^{[25]}$ nachgewiesen.

\subsubsection{Umsetzung von (Dipp) ${ }_{2} \mathrm{NacNacGaH}_{2}$ mit Methylhydrazin}

Ziel dieses Versuches war es zu untersuchen, ob Aminfunktionen reaktiv genug sind, um mit Galliumhydriden erfolgreich umgesetzt werden zu können. In diesem Fall konnte ${ }^{1} \mathrm{H}-\mathrm{NMR}$-spektroskopisch nur Edukt nachgewiesen werden. Das lässt den Schluss zu, dass Methylhydrazin nicht acid genug ist, um mit dem hydridischen Wasserstoff von (Dipp) ${ }_{2} \mathrm{NacNacGaH}_{2}$ zu reagieren.

\subsubsection{Umsetzung von (Dipp) ${ }_{2} \mathrm{NacNacGaH}_{2}$ mit tert-Butylhydroperoxid}

Hydroperoxide sind durch den elektronenziehenden Effekt eines weiteren Sauerstoffatoms acider als Alkohole. Dadurch erschien es vielversprechend, tert-Butylhydroperoxid mit (Dipp) ${ }_{2} \mathrm{NacNacGaH}_{2} \quad$ umzusetzen. ${ }^{1}$ H-NMR-spektroskopische Befunde zeigten allerdings, dass keine Reaktion stattgefunden hat. 


\subsubsection{Umsetzung von (Dipp) ${ }_{2} \mathrm{NacNacGaH}_{2}$ mit Trifluorethanol}

Fluorhaltige Alkohole sind durch den stark elektronenziehenden Effekt des Fluors in ihrer Acidität wesentlich gesteigert. Die ${ }^{1} \mathrm{H}-\mathrm{NMR}$-spektroskopische Untersuchung ergab, dass dies nicht reicht, um mit (Dipp) ${ }_{2} \mathrm{NacNacGaH}_{2} \mathrm{zu}$ reagieren. Es wurde nur Edukt nachgewiesen.

\subsection{Umsetzung von (Dipp) $)_{2} \mathrm{NacNacGaH}_{2}$ mit Säuren}

Bis auf TMSCl und ICN reagierten alle bisher eingesetzten Reagenzien nicht mit (Dipp) ${ }_{2} \mathrm{NacNacGaH}$. Die Reaktivität von organischen Säuren müsste größer sein, da ihre Protonen acider sind als die von Alkoholen oder Acetylenen.

\subsubsection{Umsetzung von (Dipp) ${ }_{2} \mathrm{NacNacGaH}_{2}$ mit Benzoesäure}

Bei dieser Reaktion wurde (Dipp) ${ }_{2} \mathrm{NacNacGaH}_{2}$ mit 2 Äquivalenten Benzoesäure $24 \mathrm{~h}$ lang bei $141{ }^{\circ} \mathrm{C}$ in $p$-Xylol umgesetzt. Dabei bildete sich ein unlöslicher Feststoff und eine sich in Lösung befindliche Substanz. Das EI-Massenspektrum des Feststoffes zeigt einen Molekülionenpeak bei m/e von 728. Das weist auf die Existenz von (Dipp) ${ }_{2} \mathrm{NacNacGa}(\mathrm{OOCPh})_{2}$ hin.

Das ${ }^{1}$ H-NMR-Spektrum der löslichen Komponente zeigt für die Verbindung typischen Signale. Diese unterscheiden sich vom Edukt und vom Liganden. Weiterhin ist ein Signal bei einer chemischen Verschiebung von 2.13 ppm zu erkennen, was auf eine NH-Funktion hinweist. Bei der Protonierung des Liganden öffnet sich der 
Vinamidinring. Es entsehen zwei NH-Funktionen, die durch die Delokalisierung der Doppelbindungen nicht unterscheidbar sind. Bei dem erhaltenen Produkt könnte es sich um $\left[(\mathrm{Dipp})_{2} \mathrm{NacNacH}_{2}\right]^{+}[\mathrm{OOCPh}]^{-}$handeln. Von der löslichen Komponente wurden Einkristalle erhalten. Der Versuch die Struktur röntgenographisch aufzuklären scheiterte. Bei dem Analyseversuch wurde allerdings keine Elektronendichte eines schweren Elementes wie Gallium gefunden, was die beschriebene These der Produktbildung unterstützt.

\subsubsection{Umsetzung von (Dipp) ${ }_{2} \mathrm{NacNacGaH}_{2}$ mit Essigsäure}

Bei dieser Reaktion wurde (Dipp) ${ }_{2} \mathrm{NacNacGaH}_{2}$ mit 2 Äquivalenten Essigsäure $12 \mathrm{~h}$ lang in siedendem Benzol umgesetzt. Dabei bildete sich ein unlöslicher Feststoff und eine sich in Lösung befindliche Substanz. Das EI-Massenspektrum des Feststoffes zeigt einen Molekülionenpeak bei m/e 604. Das weist auf die Existenz von (Dipp) ${ }_{2} \mathrm{NacNacGa}\left(\mathrm{OOCCH}_{3}\right)_{2}$ hin.

Das ${ }^{1} \mathrm{H}-\mathrm{NMR}$-Spektrum der löslichen Komponente zeigt für die Verbindung die typischen Signale. Diese unterscheiden sich vom Edukt und vom Liganden. Weiterhin ist ein breites Signal bei einer chemischen Verschiebung von 11.18 ppm zu erkennen, was auf eine $\mathrm{NH}_{2}$-Funktion hinweist. Bei dem erhaltenen Produkt könnte es sich um $\left[(\mathrm{Dipp})_{2} \mathrm{NacNaCH}_{3}\right]^{2+}\left[\mathrm{OOCCH}_{3}\right]_{2}^{-}$handeln. 


\subsubsection{Umsetzung von (Dipp) ${ }_{2} \mathrm{NacNacGaH}_{2}$ mit Trifluoressigsäure}

Bei dieser Reaktion wurde (Dipp) ${ }_{2} \mathrm{NacNacGaH}_{2}$ mit 2 Äquivalenten Trifluoressigsäure $12 \mathrm{~h}$ lang bei Raumtemperatur in Benzol umgesetzt. Dabei bildete sich ein unlöslicher Feststoff und eine sich in Lösung befindliche Substanz. Das EI-Massenspektrum des Feststoffes zeigt einen Molekülionenpeak bei m/e 712. Das weist auf die Existenz von (Dipp) $)_{2} \mathrm{NacNacGa}\left(\mathrm{OOCCF}_{3}\right)_{2}$ hin.

Das ${ }^{1}$ H-NMR-Spektrum der löslichen Komponente zeigt für die Verbindung typische Signale. Diese unterscheiden sich vom Edukt und vom Liganden. Weiterhin ist ein breites Signal bei einer chemischen Verschiebung von 10.03 ppm zu erkennen, was auf eine $\mathrm{NH}_{2}$-Funktion hinweist. Bei dem erhaltenen Produkt handelt es sich eventuell um $\left[(\mathrm{Dipp})_{2} \mathrm{NacNacH}_{3}\right]^{2+}\left[\mathrm{OOCCF}_{3}\right]_{2}^{-}$. Die Einkristallröntgenstrukturanalyse ergab die einfach protonierte Form des Vinamidins mit einem Trifluoracetat als Anion.

\subsubsection{Diskussion der Einkristallröntgenstrukturanalyse $\operatorname{von}\left[(\mathrm{Dipp})_{2} \mathrm{NacNacH}_{2}\right]^{+}\left[\mathrm{OOCCF}_{3}\right]^{-}$}

Die Kristallisation erfolgte in der monoklinen Raumgruppe P2(1)/n. Die Einkristallröntgenstrukturanalyse bestätigt die angenommene Hydrolyse des Edukts und macht den ${ }^{1}$ H-NMR-spektroskopischen Befund erklärbar. Die Säurefunktion reagierte nicht nur mit den hydridische Wasserstoffen am Gallium sondern es hydrolysierte auch die Ga-N-Bindung, was zur Abspaltung des Vinamidins führte. 
Desweiteren wurde die Aminfunktion durch die Säure protoniert und es entstand das Amid welches mit dem Säureanion zusammen kristallisierte.

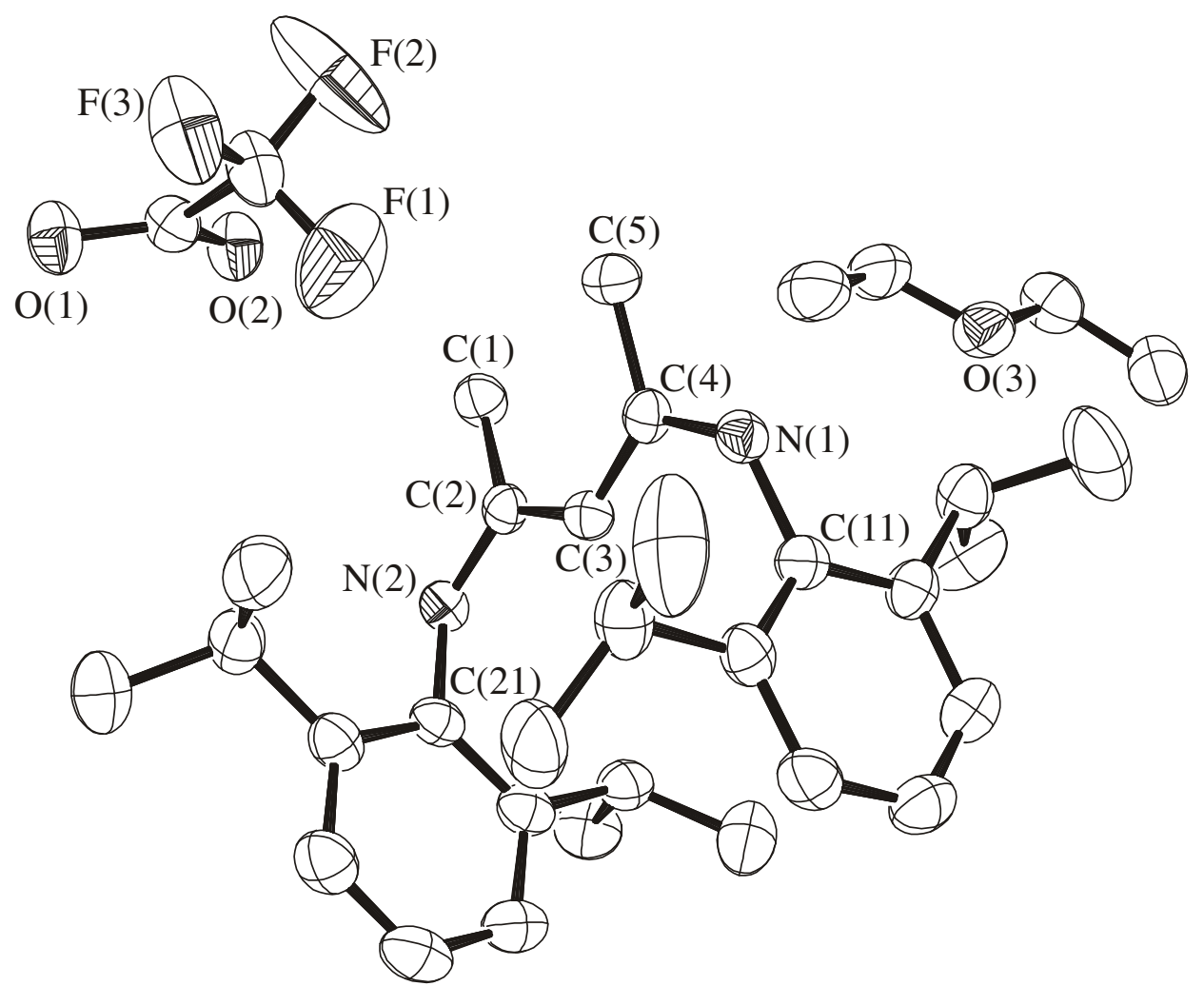

Abbildung 3.6.1.2: Struktur von $\left[(\mathrm{Dipp})_{2} \mathrm{NacNacH}_{2}\right]^{+}\left[\mathrm{OOCCF}_{3}\right]^{-}$im Kristall

Tabelle 3.6.1.2: Ausgewählte Bindungslängen $[\mathrm{pm}]$ und -winkel $\left[{ }^{\circ}\right]$ von $\left[(\mathrm{Dipp})_{2} \mathrm{NacNacH}_{2}\right]^{+}\left[\mathrm{OOCCF}_{3}\right]^{-}$.

$\begin{array}{llll}\mathrm{N}(2)-\mathrm{C}(2) & 132.9(3) & \mathrm{N}(1)-\mathrm{C}(4) & 133.5(3) \\ \mathrm{N}(2)-\mathrm{C}(21) & 144.5(3) & \mathrm{N}(1)-\mathrm{C}(11) & 144.4(3) \\ \mathrm{C}(2)-\mathrm{C}(3) & 140.0(3) & \mathrm{C}(3)-\mathrm{C}(4) & 138.9(3) \\ \mathrm{C}(1)-\mathrm{C}(2) & 150.1(3) & \mathrm{C}(4)-\mathrm{C}(5) & 150.4(3)\end{array}$




$\begin{array}{llll}\mathrm{C}(2)-\mathrm{N}(2)-\mathrm{C}(21) & 124.7(19) & \mathrm{C}(4)-\mathrm{N}(1)-\mathrm{C}(11) & 124.2(2) \\ \mathrm{N}(2)-\mathrm{C}(2)-\mathrm{C}(3) & 120.2(2) & \mathrm{N}(1)-\mathrm{C}(4)-\mathrm{C}(3) & 120.6(2) \\ \mathrm{N}(2)-\mathrm{C}(2)-\mathrm{C}(1) & 113.9(2) & \mathrm{N}(1)-\mathrm{C}(4)-\mathrm{C}(5) & 114.1(2) \\ \mathrm{C}(1)-\mathrm{C}(2)-\mathrm{C}(3) & 125.8(2) & \mathrm{C}(3)-\mathrm{C}(4)-\mathrm{C}(5) & 125.3(2) \\ \mathrm{C}(2)-\mathrm{C}(3)-\mathrm{C}(4) & 127.9(2) & & \end{array}$

Die in Tabelle 3.6.1.2 beschriebenen Bindungslängen und Winkel zeigen, dass N(1) und N(2) äquivalent sind. Die N(2)-C(2)-Bindung ist mit 132.9 pm nahezu gleichlang wie die N(1)-C(4)-Bindung mit $133.5 \mathrm{pm}$. Ebenfalls ist die C(2)-C(3)-Bindung mit $140.0 \mathrm{pm}$ ähnlich lang wie die C(3)-C(4)-Bindung mit $138.9 \mathrm{pm}$. Die beiden Doppelbindungen sind delokalisiert. An jedem Stickstoffatom befindet sich im Gegensatz zum ${ }^{1}$ H-NMR-spektroskopischen Befund ein Proton. Das Amid liegt im Kristall einfach protoniert vor. 


\section{Experimenteller Teil}

\subsection{Allgemeine Arbeitstechniken, Meßmethoden und Darstellung der Ausgangsverbindungen}

\subsubsection{Allgemeine Arbeitstechniken}

Alle Versuche wurden in ausgeheizten und im Vakuum abgekühlten Apparaturen unter einer mit $\mathrm{P}_{4} \mathrm{O}_{10}$ getrockneten Stickstoffatmosphäre durchgeführt. Die verwendeten Lösungsmittel wurden nach literaturbekannten Methoden getrocknet, mit Stickstoff gesättigt und vor Gebrauch frisch destilliert. ${ }^{[26]}$

\subsubsection{Meßmethoden}

Schmelzpunkte wurden in abgeschlossenen Glaskapillaren mit dem Gerät Büchi Melting Point B-540 bestimmt.

NMR-Spektren wurden mit den Geräten Bruker AM 200, Bruker AC 250 und Bruker MLS 400 aufgenommen.

Als externe Standards wurden Tetramethylsilan $\left({ }^{1} \mathrm{H}-,{ }^{13} \mathrm{C}-,{ }^{29} \mathrm{Si}-\mathrm{NMR}\right)$ und $\mathrm{C}_{6} \mathrm{~F}_{6}$ $\left({ }^{19}\right.$ F-NMR) verwendet. Heterokern-NMR-Spektren wurden ${ }^{1} \mathrm{H}$-breitbandentkoppelt aufgenommen. Lösungsmittel und Betriebsfrequenzen sind in den Versuchsbeschreibungen aufgeführt. Die Multiplizitäten werden wie folgt angegeben: $\mathrm{s}=$ Singulett, $\mathrm{d}=$ Dublett, $\mathrm{t}=$ Triplett, $\mathrm{q}=$ Quartett, sept $=$ Septett, $\mathrm{m}=$ Multiplett . 
Die chemischen Verschiebungen $\delta$ sind in ppm angegeben, wobei negative Vorzeichen eine Hochfeldverschiebung und positive Vorzeichen eine Tieffeldverschiebung bezogen auf den jeweiligen Standard bedeuten. Die Messungen erfolgten bei Raumtemperatur.

Massenspektren wurden mit den Geräten Varian MAT CH5, Finnigan MAT 95 und Finnigan MAT System 8230 aufgenommen. In allen Fällen wurde ElektronenstoßIonisation (EI) bei $70 \mathrm{eV}$ als Ionisationsmethode verwendet. Es wird der stärkste $\mathrm{zu}$ einer Isotopenverteilung gehörende Peak mit seiner relativen prozentualen Intensität angegeben.

IR-Spektren wurden auf einem BIO-RAD Digilab FTS 7 Spektrometer aufgenommen. Alle Substanzen wurden als Nujolverreibung zwischen KBr-Platten gemessen. Die Bandenintensitäten sind wie folgt abgekürzt: sst $=$ sehr stark, $s t=$ stark, $\mathrm{m}=$ mittel, $\mathrm{s}=$ schwach. Es werden nur mittelstarke, starke, sehr starke und charakteristische Banden angegeben.

Elementaranalysen wurden im Analytischen Labor des Institutes für Anorganische Chemie der Georg-August-Universität Göttingen durchgeführt.

Einkristall-Röntgenstrukturanalysen wurden auf einem Stoe-Siemens-AEDVierkreisdiffraktometer oder auf einem Stoe-Siemens-Huber-Vierkreisdiffraktometer, ausgerüstet mit einem Siemens-Smart-CCD-Flächenzähler, an einem schockgekühlten Kristall im Öltröpfchen ${ }^{[27]}$ mit monochromatisierter 
Mo-K $\mathrm{K}_{\alpha}$-Strahlung $(\lambda=71.073 \mathrm{pm})$ durchgeführt. Die Lösung der Strukturen erfolgte mit direkten Methoden (SHELXS-97), ${ }^{[28]}$ die Verfeinerung gegen $F^{2}$ unter Verwendung aller Daten (SHELXL-97). ${ }^{[29]}$ Alle Atome mit Ausnahme der Wasserstoffatome wurden anisotrop verfeinert. Die Positionierung der Wasserstoffatome erfolgte nach dem Reitermodell. Am Gallium gebundene Wasserstoffe wurden ebenfalls anisotrop verfeinert.

\subsubsection{Darstellung der Ausgangsverbindungen}

Die Ausgangsverbindungen waren im Handel erhältlich oder wurden nach folgenden Literaturvorschriften dargestellt:

Di-iso-propylarduengocarben, ${ }^{[30]}$ Dimesitylarduengocarben, ${ }^{[31]}(\mathrm{Dipp})_{2} \mathrm{NacNacH},{ }^{[25]}$ (Dipp) ${ }_{2} \mathrm{NacNacGaCl}_{2},{ }^{[16]}$ (Dipp) ${ }_{2} \mathrm{NacNacGaI}_{2},{ }^{[16]} \mathrm{H}-\mathrm{C} \equiv \mathrm{C}-\mathrm{S}-n-\mathrm{Bu},{ }^{[32]} \mathrm{H}-\mathrm{C} \equiv \mathrm{C}-\mathrm{SO}_{2}-$ $\left(\mathrm{p}-\mathrm{MeC}_{6} \mathrm{H}_{4}\right),{ }^{[33]} \quad \mathrm{GaCl}_{3},{ }^{[34,35]} \quad \mathrm{GaBr}_{3},{ }^{[36]} \quad \mathrm{GaI}_{3},{ }^{[37]} \quad$ TMSacetylen, ${ }^{[13,14,15]}$ (Dipp) ${ }_{2} \mathrm{NacNacLi} \cdot \mathrm{Et}_{2} \mathrm{O},{ }^{[38]} \mathrm{LiGaH}_{4},{ }^{[5]} \mathrm{GaH}_{3} \cdot \mathrm{NMe}_{3},{ }^{[6]} \mathrm{LiHBEt}_{3},{ }^{[39,40]} \mathrm{Me}_{3} \mathrm{SnF}^{[41]}$

\subsubsection{Modifizierte Darstellung von $\mathrm{GaH}_{3} \cdot \mathrm{NMe}_{3}$}

$12.00 \mathrm{~g}$ (1.500 mol) LiH-Pulver werden zwei Stunden lang in einer Kugelmühle gemahlen. Das gemahlene Hydrid wird in $800 \mathrm{ml}$ Diethylether suspendiert. $16.50 \mathrm{~g}$ (93.70 mmol) frisch sublimiertes $\mathrm{GaCl}_{3}$ werden bei ca. $-40{ }^{\circ} \mathrm{C}$ in $200 \mathrm{ml}$ Diethylether gelöst, wobei sich bei einer stark exothermen Reaktion das Etherat bildet. Bei $0{ }^{\circ} \mathrm{C}$ wird unter starkem Rühren die $\mathrm{GaCl}_{3}$-Lösung zur LiH-Suspension getropft. Eine Stunde nach der Zugabe wird die Kühlung entfernt und weiter $24 \mathrm{~h}$ gerührt. Die 
entstandene $\mathrm{LiGaH}_{4}$-Lösung wird durch Filtration vom entstandenem $\mathrm{LiCl}$ und dem überschüssigen LiH getrennt. Die Ausbeute wird auf $75 \%$ geschätzt. $\mathrm{Zu}$ dieser Lösung wird bei $0{ }^{\circ} \mathrm{C}$ ein Äquivalent $(6.72 \mathrm{~g}, 70.27 \mathrm{mmol})$ getrocknetes $\mathrm{NMe}_{3} \cdot \mathrm{HCl}$ mittels Schneckenradfeststofftrichter zugeführt. Bei vorzeitiger Beendigung der $\mathrm{H}_{2}$-Entwicklung ist gegebenenfalls die Zugabe abzubrechen (Ausbeute von $\mathrm{LiGaH}_{4}$ geringer als $75 \%$ ). Das Reaktionsgemisch ist noch eine Stunde lang nach Beendigung der Zugabe bei Raumtemperatur zu rühren. Danach wird die Lösung mit einem Kühlaggregat auf $-45^{\circ} \mathrm{C}$ gekühlt. Nach dem Erreichen dieser Temperatur wird mit dem Abdestillieren des Ethers im Vakuum begonnen. Dieser Prozess dauert ca. 12 h. Das zurückgebliebene Reaktionsprodukt wird bei Raumtemperatur sublimiert. Dies geschieht direkt aus dem Reaktionsgefäß über Umfüllbögen in ein breites Schlenkrohr. Dabei ist darauf zu achten, dass große Querschnitte benutzt werden. Das Schlenkrohr ist ein Gefäß, das aus zwei parallelen Glasrohren besteht. Ein breites mit einem großen Schliff an der Oberseite und ein schmales mit einem Hahn an der Oberseite. Beide Rohre sind an der Unterseite miteinander verbunden. An das schmale Rohr wird Vakuum angelegt (Schlenkhahn) und in das breite Rohr wird hineinsublimiert. Bei der Sublimation ist das Schlenkrohr mit einem Kühlaggregat auf $-45{ }^{\circ} \mathrm{C}$ zu kühlen. Die Ausbeute beträgt ca. $43 \%$ (6 g, $\left.40 \mathrm{mmol}\right)$ bezogen auf das eingesetzte $\mathrm{GaCl}_{3}$.

${ }^{1}$ H-NMR (300.13 MHz, C ${ }_{6} \mathrm{D}_{6}$ ): $\delta 2.00$ (s, $9 \mathrm{H}, \mathrm{CH}_{3}$ ), 4.97 (s (br), $3 \mathrm{H}, \mathrm{Ga} H$ ) ppm.

IR (Nujol, $\mathrm{cm}^{-1}$ ) $\tilde{v}:$ 3160(s), 1906 (s), 1837(st), 1631(st), 1307(s), 1272(m), 1153(m), 972(s), 739(sst), 618(s), 486(s). 


\subsection{Umsetzung von $\mathrm{GaH}_{3} \cdot \mathrm{NMe}_{3}$ mit Acetylenen}

\subsubsection{Darstellung von $\mathrm{Me}_{3} \mathrm{~N} \cdot \mathrm{Ga}(-\mathrm{C} \equiv \mathrm{C}-\mathrm{Ph})_{3}$}

$\mathrm{Zu} 3.00 \mathrm{~g}, 22.7 \mathrm{mmol} \mathrm{GaH} \cdot \mathrm{NMe}_{3}$ gelöst in $150 \mathrm{ml}$ Toluol werden 10 Äquivalente (23.1 g, $227 \mathrm{mmol})$ Phenylacetylen getropft. Das Reaktionsgemisch wird $12 \mathrm{~h}$ lang bei einer Ölbadtemperatur von $55^{\circ} \mathrm{C}$ gerührt. Nachdem die Lösung auf Raumtemperatur abgekühlt ist, werden alle flüchtigen Bestandteile im Vakuum abdestilliert. Der Rückstand wird erneut in $50 \mathrm{ml}$ Toluol gelöst und filtriert. Die Lösung wird auf ca. 10 bis $20 \mathrm{ml}$ eingeengt, wobei das Produkt ausfällt. Durch Erhitzen löst sich das Produkt wieder vollständig und kristallisiert bei $4{ }^{\circ} \mathrm{C}$ mit einer Ausbeute von $71 \%$ (6.96 g, $16.1 \mathrm{mmol})$.

Schmelzpunkt: $151^{\circ} \mathrm{C}$.

${ }^{1}$ H-NMR $\left(500.13 \mathrm{MHz}, \mathrm{C}_{6} \mathrm{D}_{6}\right): \delta 2.28\left(\mathrm{~s}, 9 \mathrm{H}, \mathrm{CH}_{3}\right), 6.97-6.95(\mathrm{~m}, 9 \mathrm{H}, m$ - und $\left.p-\mathrm{C}_{6} H_{5}\right), 7.51-7.49\left(\mathrm{~m}, 6 \mathrm{H}, o-\mathrm{C}_{6} H_{5}\right) \mathrm{ppm}$.

${ }^{13}$ C-NMR (125.75 MHz, $\left.\mathrm{C}_{6} \mathrm{D}_{6}\right): \delta 47.3\left(\mathrm{CH}_{3}\right), 99.2(\mathrm{br}, \mathrm{Ga}-C), 107.2\left(\mathrm{C}_{6} \mathrm{H}_{5}-C\right)$, 132.3, 128.4, 128.0, 125.1 (arom. C) ppm.

MS: m/e (\%) $431(\mathrm{M}, 30), 372\left(\mathrm{M}-\mathrm{NMe}_{3}, 100\right)$.

IR (Nujol, $\mathrm{cm}^{-1}$ ) $\tilde{v}:$ 3053(s), 2137 (st), 1981(s), 1960(s), 1890(s), 1815(s), 1683(s), 1597(m), 1570(m), 1487(s), 1303(s), 1277(s), 1249(m), 1210(st), 1176(m), 1158(s), 
1105(m), 1070(m), 1024(m), 996(s), 920(st), 848(s), 823(m), 811(m), 796(st), 764(st), 756(st), 725(m), 692(st), 623(s), 568(st), 535(st), 520(st), 422(m), 384(st).

Elementaranalyse: $\quad$ ber. C 75.03, H 5.60, Ga 16.13, N 3.24; gef. C 75.10, H 5.60, Ga 15.30, N 3.33.

\subsubsection{Darstellung von $\mathrm{Me}_{3} \mathrm{~N} \cdot \mathrm{Ga}(-\mathrm{C} \equiv \mathrm{C}-\mathrm{TMS})_{3}$}

$\mathrm{Zu} 1.00 \mathrm{~g}$ (7.58 mmol) $\mathrm{GaH}_{3} \cdot \mathrm{NMe}_{3}$ gelöst in $50 \mathrm{ml}$ Toluol werden 9 Äquvivalente (6.70 g, $0.0682 \mathrm{~mol})$ Trimethylsilylacetylen getropft. Die Lösung wird $1 \mathrm{~d}$ lang bei $55^{\circ} \mathrm{C}$ gerührt. Nach dem Abkühlen auf Raumtemperatur werden alle flüchtigen Bestandteile im Vakuum destillativ entfernt. Der feste Rückstand wird in $50 \mathrm{ml}$ Toluol aufgenommen und filtriert. Die erhaltene Lösung wird auf $15 \mathrm{ml}$ eingeengt. Das dabei als Feststoff anfallende Produkt wird durch kurzes Erwärmen wieder gelöst. Die erhaltene Zielverbindung kristallisiert bei Raumtemperatur mit einer Ausbeute von $44 \%(1.40 \mathrm{~g}, 3.35 \mathrm{mmol})$.

Schmelzpunkt: $154^{\circ} \mathrm{C}$.

${ }^{1}$ H-NMR (300.13 MHz, C $\left.6 \mathrm{D}_{6}\right): \delta 0.12\left(\mathrm{~s}, 27 \mathrm{H}, \mathrm{SiCH}_{3}\right), 1.96\left(\mathrm{~s}, 9 \mathrm{H}, \mathrm{NCH}_{3}\right) \mathrm{ppm}$.

${ }^{13}$ C-NMR $\left(125.75 \mathrm{MHz}, \mathrm{C}_{6} \mathrm{D}_{6}\right): \delta 0.06\left(\mathrm{SiCH}_{3}\right), 46.73\left(\mathrm{NCH}_{3}\right), 89.71(\mathrm{Ga}-\mathrm{C}), 93.88$ (TMS-C) ppm.

${ }^{29} \mathrm{Si}-\mathrm{NMR}\left(59.68 \mathrm{MHz}, \mathrm{CDCl}_{3}\right): \delta-19.64\left(\mathrm{C} \equiv \mathrm{C}-\mathrm{SiMe}_{3}\right) \mathrm{ppm}$. 
MS: m/e (\%) 419 (M, 9), $404(\mathrm{M}-\mathrm{Me}, 33), 359\left(\mathrm{M}-\mathrm{NMe}_{3}, 16\right)$,

$344\left(\mathrm{M}-\mathrm{NMe}_{3}-\mathrm{Me}, 100\right)$.

Elementaranalyse: $\quad$ ber. C 51.42, H 8.63, Ga 16.58, N 3.33, Si 20.04;

gef. C 49.88, H 8.31, Ga 16.30, N 3.45, Si 19.64.

\subsubsection{Darstellung von $\mathrm{Me}_{3} \mathrm{~N} \cdot \mathrm{Ga}(-\mathrm{C} \equiv \mathrm{C}-\mathrm{S}-n-\mathrm{Bu})_{3}$}

$\mathrm{Zu} 1.00 \mathrm{~g}(7.58 \mathrm{mmol}) \mathrm{Me}_{3} \mathrm{~N} \cdot \mathrm{GaH}_{3}$ gelöst in $50 \mathrm{ml}$ Toluol werden 3 Äquvivalente $(2.59 \mathrm{~g}, 0.0227 \mathrm{~mol})$ des Acetylens (in $30 \mathrm{ml}$ Toluol gelöst) getropft. Die Lösung wird $1 \mathrm{~d}$ lang bei RT gerührt. Danach werden alle flüchtigen Bestandteile im Vakuum destillativ entfernt. Der feste Rückstand wird in $30 \mathrm{ml}$ Diethylether aufgenommen und filtriert. Nach dem Entfernen des Diethylethers wird die ölige Zielverbindung mit einer Ausbeute von $48 \%$ erhalten $(1.70 \mathrm{~g}, 3.64 \mathrm{mmol})$.

${ }^{1} \mathbf{H}$-NMR $\left(300.13 \mathrm{MHz}, \mathrm{CDCl}_{3}\right): \delta 0.87\left(\mathrm{~m}, 9 \mathrm{H}, \mathrm{S}-\mathrm{CH}_{2}-\mathrm{CH}_{2}-\mathrm{CH}_{2}-\mathrm{CH}_{3}\right), 1.41(\mathrm{~m}$, $\left.6 \mathrm{H}, \mathrm{S}-\mathrm{CH}_{2}-\mathrm{CH}_{2}-\mathrm{CH}_{2}-\mathrm{CH}_{3}\right), 1.68\left(\mathrm{~m}, 6 \mathrm{H}, \mathrm{S}-\mathrm{CH}_{2}-\mathrm{CH}_{2}-\mathrm{CH}_{2}-\mathrm{CH}_{3}\right), 2.47$ (s, $9 \mathrm{H}$, $\left.\mathrm{NCH}_{3}\right), 2.70\left(\mathrm{~m}, 6 \mathrm{H}, \mathrm{S}-\mathrm{CH}_{2}-\mathrm{CH}_{2}-\mathrm{CH}_{2}-\mathrm{CH}_{3}\right) \mathrm{ppm}$.

MS: m/e (\%) $393\left(\mathrm{M}-\mathrm{NMe}_{3}-\mathrm{Me}, 2\right), 366$ (M - $\mathrm{NMe}_{3}-n$-Propyl, 2), $182(\mathrm{Ga}-\mathrm{C} \equiv \mathrm{C}-\mathrm{S}-n-\mathrm{Bu}, 15), 92(\mathrm{Ga}-\mathrm{C} \equiv \mathrm{C}, 100)$. 


\subsubsection{Darstellung von $\mathrm{NMe}_{3} \cdot \mathrm{Ga}\left(-\mathrm{C} \equiv \mathrm{C}-\mathrm{SO}_{2}-\left(p-\mathrm{MeC}_{6} \mathrm{H}_{4}\right)\right)_{3}$}

$\mathrm{Zu} 1.00 \mathrm{~g}$ (7.58 mmol) $\mathrm{GaH}_{3} \cdot \mathrm{NMe}_{3}$ gelöst in $50 \mathrm{ml}$ Toluol werden 3 Äquvivalente $(4.09 \mathrm{~g}, 0.0227 \mathrm{~mol})$ des Acetylens (gelöst in $30 \mathrm{ml}$ Toluol) getropft. Die Lösung wird $1 \mathrm{~d}$ lang bei RT gerührt. Danach werden alle flüchtigen Bestandteile im Vakuum destillativ entfernt. Der feste Rückstand wird in $30 \mathrm{ml}$ Diethylether aufgenommen und filtriert. Nach dem Entfernen des Diethylethers wird die ölige Zielverbindung mit einer Ausbeute von $63 \%$ erhalten $(3.18 \mathrm{~g}, 4.77 \mathrm{mmol})$.

${ }^{1}$ H-NMR $\left(300.13 \mathrm{MHz}, \mathrm{CDCl}_{3}\right): \delta 2.42\left(\mathrm{~s}, 9 \mathrm{H}, \mathrm{NCH}_{3}\right), 2.58\left(\mathrm{~s}, 9 \mathrm{H}, p-\mathrm{CH}_{3}-\mathrm{Ph}\right), 7.33$ (m, $6 \mathrm{H}, m$-arom), $7.78(\mathrm{~m}, 6 \mathrm{H}, o$-arom) ppm.

MS: m/e (\%) $202(\mathrm{Ga}-\mathrm{C} \equiv \mathrm{C}-\mathrm{S}-\mathrm{Ph}, 8), 150\left(\mathrm{NMe}_{3} \mathrm{Ga}-\mathrm{C} \equiv \mathrm{C}, 55\right), 114\left(\mathrm{GaNMe}_{2}, 45\right)$, $57\left(\mathrm{NMe}_{2} \mathrm{CH}, 100\right)$.

\subsection{Austausch von $\mathrm{NMe}_{3}$ durch Arduengocarbene am $\mathrm{GaH}_{3}$}

\subsubsection{Darstellung von $\mathrm{H}_{3} \mathrm{Ga} \cdot \mathrm{C}(\mathrm{N}(\text { iso-propyl }))_{2} \mathrm{C}_{2} \mathrm{H}_{2}$}

$\mathrm{Zu} 1.00 \mathrm{~g}$ (7.58 mmol) $\mathrm{Me}_{3} \mathrm{~N} \cdot \mathrm{GaH}_{3}$ gelöst in $50 \mathrm{ml}$ Toluol wird 1 Äquivalent iso-Propylarduengocarben $(7.58 \mathrm{mmol}, 1.15 \mathrm{~g})$ gelöst in $20 \mathrm{ml}$ Toluol getropft. Die Lösung wird $1 \mathrm{~d}$ lang bei Raumtemperatur gerührt. Die bei der Reaktion entstehenden unlöslichen Zersetzungsprodukte werden abfiltriert. Das Lösungsmittel wird im 
Vakuum abdestilliert. Das erhaltene Rohprodukt kristallisiert in Toluol bei $-27{ }^{\circ} \mathrm{C}$ mit einer Ausbeute von $10 \%(0.17 \mathrm{~g}, 0.76 \mathrm{mmol})$.

Schmelzpunkt: $127^{\circ} \mathrm{C}$.

${ }^{1}$ H-NMR (300.13 MHz, $\left.\mathrm{C}_{6} \mathrm{D}_{6}\right): \delta 0.91\left(\mathrm{~d}, J=6.7 \mathrm{~Hz}, 12 \mathrm{H}, \mathrm{C}\left(\mathrm{CH}_{3}\right)_{2}\right), 4.55$ (s br, $3 \mathrm{H}$, $\left.\mathrm{GaH}_{3}\right), 5.18$ (sept, $\left.2 \mathrm{H}, \mathrm{CH}\left(\mathrm{CH}_{3}\right)_{2}\right), 6.23(\mathrm{~s}, 2 \mathrm{H}, \mathrm{HC}=\mathrm{CH}) \mathrm{ppm}$.

\subsubsection{Darstellung von $\mathrm{H}_{3} \mathrm{Ga} \cdot \mathrm{C}(\mathrm{NMes})_{2} \mathrm{C}_{2} \mathrm{H}_{2}$}

$\mathrm{Zu} 1.00 \mathrm{~g}$ (7.58 mmol) $\mathrm{Me}_{3} \mathrm{~N} \cdot \mathrm{GaH}_{3}$ gelöst in $50 \mathrm{ml}$ Benzol wird 1 Äquivalent Mesitylarduengocarben (7.58 mmol, $2.02 \mathrm{~g}$ ) gelöst in $20 \mathrm{ml}$ Benzol getropft. Die Lösung wird $1 \mathrm{~d}$ lang bei Raumtemperatur gerührt. Das schwer lösliche Produkt bildet im Verlauf der Reaktion einen Niederschlag. Das Lösungsmittel wird im Vakuum abdestilliert. Die Ausbeute beträgt $96 \%$ (2.47 g, 7.28 mmol).

Schmelzpunkt: $247^{\circ} \mathrm{C}$.

${ }^{1} \mathbf{H}-\mathbf{N M R}\left(200.3 \mathrm{MHz}, \mathrm{CDCl}_{3}\right): \delta 2.33\left(\mathrm{~s}, 12 \mathrm{H}, o-\mathrm{CH}_{3}\right), 2.60\left(\mathrm{~s}, 6 \mathrm{H}, p-\mathrm{CH}_{3}\right), 3.05(\mathrm{~s}$ br, $\left.3 \mathrm{H}, \mathrm{Ga} H_{3}\right), 7.39$ (s, $\left.4 \mathrm{H}, \mathrm{Ar}-H\right), 7.51(\mathrm{~s}, 2 \mathrm{H}, H \mathrm{C}=\mathrm{C} H) \mathrm{ppm}$.

${ }^{13}$ C-NMR $\left(50.3 \mathrm{MHz}, \mathrm{CDCl}_{3}\right): \delta 17.52\left(o-\mathrm{CH}_{3}\right), 21.16\left(p-\mathrm{CH}_{3}\right), 122.68(\mathrm{HC}=C \mathrm{H})$, 129.13(Ar-C), $134.78(\mathrm{Ar}-C), 139.50(\mathrm{Ar}-C) \mathrm{ppm}$.

MS: m/e (\%) $376(\mathrm{M}, 35), 375$ (M - H, 100), 361 (M - Me, 12). 
IR (Nujol, $\mathrm{cm}^{-1}$ ) $\tilde{v}:$ 3621(s), 3160(s), 2030(s), 1797(sst), 1683(s), 1610(s), 1305(m), 1235(s), 1155(m), 1112(s), 1081(s), 1035(m), 964(m), 932(s), 895(m), 856(st), 723(sst), 645(s), 571(m), 550(m), 513(m), 492(s), 455(s).

Elementaranalyse: ber.: C 66.87, H 7.22, Ga 18.49, N 7.43; gef.: C 66.96, H 7.23, Ga 18.25, N 7.51.

\subsection{Umsetzung von $\mathrm{GaH}_{3}$-Arduengocarbenadukt mit TMSI}

\subsubsection{Umsetzung von $\mathrm{H}_{3} \mathrm{Ga} \cdot \mathrm{C}(\mathrm{NMes})_{2} \mathrm{C}_{2} \mathrm{H}_{2}$ mit 1,2 und 3 Äq. TMSI}

$0.20 \mathrm{~g}(0.59 \mathrm{mmol}) \mathrm{H}_{3} \mathrm{Ga} \cdot \mathrm{C}(\mathrm{NMes})_{2} \mathrm{C}_{2} \mathrm{H}_{2}$ gelöst in $50 \mathrm{ml}$ Benzol werden jeweils mit einem, zwei und drei Äquivalenten Trimethysilyliodid versetzt und $24 \mathrm{~h}$ lang bei Raumtemperatur gerührt. Danach werden alle flüchtigen Bestandteile im Vakuum entfernt. Bei einem und zwei Äquivalenten wird ein Gemisch aus $\mathrm{H}_{3} \mathrm{Ga} \cdot \mathrm{C}(\mathrm{NMes})_{2} \mathrm{C}_{2} \mathrm{H}_{2}$ und $\mathrm{I}_{3} \mathrm{Ga} \cdot \mathrm{C}(\mathrm{NMes})_{2} \mathrm{C}_{2} \mathrm{H}_{2}$ erhalten. Bei drei $\ddot{\text { Äquivalenten bildet }}$ sich ausschließlich $\mathrm{I}_{3} \mathrm{Ga} \cdot \mathrm{C}(\mathrm{NMes})_{2} \mathrm{C}_{2} \mathrm{H}_{2}$ mit einer Ausbeute von $92 \%(0.39 \mathrm{~g}$, $0.54 \mathrm{mmol})$.

Analytische Daten bei Umsetzung mit 3 Äquivalenten TMSI:

Schmelzpunkt: $280^{\circ} \mathrm{C}$.

${ }^{1}$ H-NMR (300.13 MHz, $\mathrm{C}_{6} \mathrm{D}_{6}$ ): $\delta 1.79\left(\mathrm{~s}, 6 \mathrm{H}, p-\mathrm{CH}_{3}\right), 2.04\left(\mathrm{~s}, 12 \mathrm{H}, o-\mathrm{CH}_{3}\right), 6.71(\mathrm{~s}$, $4 \mathrm{H}, \mathrm{Ar}-H), 5.77(\mathrm{~s}, 2 \mathrm{H}, H \mathrm{C}=\mathrm{C} H) \mathrm{ppm}$. 
${ }^{13}$ C-NMR $\quad\left(125.75 \quad \mathrm{MHz}, \quad \mathrm{C}_{6} \mathrm{D}_{6}\right): \quad \delta \quad 18.93 \quad\left(o-\mathrm{CH}_{3}\right), \quad 21.17 \quad\left(p-\mathrm{CH}_{3}\right), \quad 68.01$ $(\mathrm{Ga}-C-\mathrm{N}) 124.27(\mathrm{HC}=C \mathrm{H}), 130.13(\mathrm{Ar}-C), 135.63(\mathrm{Ar}-C), 141.75(\mathrm{Ar}-C) \mathrm{ppm}$.

MS: m/e (\%) 753 (M, 1), 627 (M - I, 100), 499 (M - 2I, 5), 373 (M - 3I).

IR (Nujol, $\mathrm{cm}^{-1}$ ) $\tilde{v}:$ 3618(s), 3149(s), 2405(s), 1733(sst), 1664(s), 1603(s), 1302(m), 1263(m), 1154(s), 1121(s), 1073(s), 1031(m), 965(s), 932(s), 892(s), 848(s), 802 (s), 769 (s), 722(sst), 640(s), 565(s), 469(s).

Elementaranalyse: ber.: C 33.41, H 3.20, Ga 9.24, I 50.43, N 3.71

gef.: C 33.56, H 3.27, Ga 9.12, I 49.86, N 3.85.

\subsection{Darstellung von (Dipp) ${ }_{2}$ NacNacGa-Halogeniden und Hydriden}

\subsubsection{Darstellung von (Dipp) ${ }_{2} \mathrm{NacNacGaBr}_{2}$}

$1.00 \mathrm{~g}$ (3.23 mmol) $\mathrm{GaBr}_{3}$ wird bei $-78^{\circ} \mathrm{C}$ in $40 \mathrm{ml}$ Diethylether gelöst. Dazu werden ebenfalls bei $-78 \quad{ }^{\circ} \mathrm{C} \quad 1.61 \mathrm{~g} \quad(3.23 \mathrm{mmol}) \quad \mathrm{NacNacLi} \cdot \mathrm{Et}_{2} \mathrm{O}$, gelöst in $20 \mathrm{ml}$ Diethylether, getropft. Nachdem die Zugabe beendet ist, wird die Reaktionslösung $12 \mathrm{~h}$ lang gerührt, wobei diese sich langsam auf Raumtemperatur erwärmt. Danach wird das Lösungsmittel im Vakuum destillativ entfernt. Der feste Rückstand wird in $50 \mathrm{ml} n$-Hexan aufgenommen und filtriert. Das $n$-Hexan wird ebenfalls im Vakuum entfernt. Das erhaltene Rohprodukt wird in $30 \mathrm{ml}$ Diethylether gelöst, und kristallisiert bei $-27{ }^{\circ} \mathrm{C}$ mit einer Ausbeute von $82 \%$ (1.71 g, $\left.2.65 \mathrm{mmol}\right)$. 
Schmelzpunkt: $236^{\circ} \mathrm{C}$.

${ }^{1}$ H-NMR $\left(200.13 \mathrm{MHz}, \mathrm{CDCl}_{3}\right): \delta 1.20\left(\mathrm{~d}, J=6.8 \mathrm{~Hz}, 12 \mathrm{H}, \mathrm{CH}\left(\mathrm{CH}_{3}\right)_{2}, 1.31(\mathrm{~d}\right.$, $J=6.8 \mathrm{~Hz}, 12 \mathrm{H}, \mathrm{CH}\left(\mathrm{CH}_{3}\right)_{2}, 1.90\left(\mathrm{~s}, 6 \mathrm{H}, \mathrm{CH}_{3}\right), 3.37$ (sept, J=6.8 Hz, $4 \mathrm{H}$, $\mathrm{CH}\left(\mathrm{CH}_{3}\right)_{2}, 5.31(\mathrm{~s}, 1 \mathrm{H}, \mathrm{CH}), 7.20-7.30(\mathrm{~m}, 6 \mathrm{H}, \mathrm{ArH}) \mathrm{ppm}$.

${ }^{13}$ C-NMR $\left(200.13 \mathrm{MHz}, \mathrm{CDCl}_{3}\right): \delta 24.15\left(\mathrm{NC}\left(\mathrm{CH}_{3}\right)\right), 24.57\left(\mathrm{CH}\left(\mathrm{CH}_{3}\right)\right), 25.54$ $\left(\mathrm{CH}\left(\mathrm{CH}_{3}\right)_{2}\right), 28.58\left(\mathrm{CH}\left(\mathrm{CH}_{3}\right)_{2}\right), 97.52(\gamma-C), 124.51(\mathrm{ArC}), 127.86(\mathrm{ArC}), 138.19$ $(\mathrm{ArC}), 144.50(\mathrm{ArC}), 171.18(\mathrm{NC}) \mathrm{ppm}$.

MS: m/e (\%) $646(\mathrm{M}, 38), 567(\mathrm{M}-\mathrm{Br}, 100), 486(\mathrm{M}-2 \mathrm{Br}, 7)$.

IR (Nujol, $\mathrm{cm}^{-1}$ ) $\tilde{v}: 1877$ (s), 1654 (m), 1522 (st), $1261(\mathrm{~m}), 1149$ (m), 1099 (sst), 1021 (sst), 934 (s), 875 (s), 799 (st), 722 (st), 638 (s), 535 (m).

Elementaranalyse: $\quad$ ber.: C 53.82, H 6.39, Br 24.69, Ga 10.77, N 4.33 gef.: C 53.91, H 6.42, Br 24.32, Ga 10.54, N 4.39.

\subsubsection{Darstellung von (Dipp) ${ }_{2} \mathrm{NacNacGaF}_{2}$}

$1.00 \mathrm{~g}(1.35 \mathrm{mmol}) \mathrm{NacNacGaI}_{2}$ gelöst in $60 \mathrm{ml}$ Benzol werden mit 2 Äquivalenten $(0.494 \mathrm{~g}, \quad 2.70 \mathrm{mmol}) \mathrm{Me}_{3} \mathrm{SnF}$ versetzt. Die Suspension wird solange bei Raumtemperatur gerührt bis sich eine klare Lösung bildet (4 Wochen). Alle flüchtigen Bestandteile werden im Vakuum destillativ entfernt. Der verbliebene 
Feststoff wird in $30 \mathrm{ml}$ Diethylethter aufgenommen. Nach dem Filtrieren der Lösung kristallisiert das Produkt bei $4{ }^{\circ} \mathrm{C}$ mit einer Ausbeute von $90 \%$ (0.63 g, $\left.1.2 \mathrm{mmol}\right)$.

Schmelzpunkt: $237^{\circ} \mathrm{C}$.

${ }^{1}$ H-NMR $\left(500.13 \mathrm{MHz}, \mathrm{C}_{6} \mathrm{D}_{6}\right): \delta 1.09\left(\mathrm{~d}, J=6.8 \mathrm{~Hz}, 12 \mathrm{H}, \mathrm{CH}\left(\mathrm{CH}_{3}\right)_{2}, 1.44(\mathrm{~d}\right.$, $J=6.8 \mathrm{~Hz}, 12 \mathrm{H}, \mathrm{CH}\left(\mathrm{CH}_{3}\right)_{2}, 1.52\left(\mathrm{~s}, 6 \mathrm{H}, \mathrm{CH}_{3}\right), 3.30$ (sept, J=6.8 Hz, $4 \mathrm{H}$, $\mathrm{CH}\left(\mathrm{CH}_{3}\right)_{2}, 4.87(\mathrm{~s}, 1 \mathrm{H}, \mathrm{CH}), 7.05-7.13(\mathrm{~m}, 6 \mathrm{H}, \mathrm{ArH}) \mathrm{ppm}$.

${ }^{13} \mathrm{C}-\mathrm{NMR} \quad\left(500.13 \mathrm{MHz}, \mathrm{C}_{6} \mathrm{D}_{6}\right): \delta 20.74\left(\mathrm{NC}\left(\mathrm{CH}_{3}\right)\right), 23.39\left(\mathrm{CH}\left(\mathrm{CH}_{3}\right)_{2}\right), 24.60$ $\left(\mathrm{CH}\left(\mathrm{CH}_{3}\right)_{2}\right), 28.60\left(\mathrm{CH}\left(\mathrm{CH}_{3}\right)_{2}\right), 95.75(\gamma-C), 138.02(\mathrm{ArC}), 141.24(\mathrm{ArC}), 142.76$ $(\mathrm{ArC}), 144.38(\mathrm{ArC}), 172.36(\mathrm{NC}) \mathrm{ppm}$.

${ }^{19}$ F-NMR $\left(188.28 \mathrm{MHz}, \mathrm{C}_{6} \mathrm{D}_{6}\right): \delta 38.34(\mathrm{GaF}) \mathrm{ppm}$.

MS: m/e (\%) 524 (M, 13), 505 ( M - F, 100), 486 (M - 2 F, 33).

Elementaranalyse: $\quad$ ber. C 66.30, H 7.87, F 7.23, Ga 13.27, N 5.33;

gef. C 66.42, H 7.90, F 7.19, Ga 13.10, N 5.38. 


\subsection{3.a Darstellung von (Dipp) $)_{2} \mathrm{NacNacGaH}_{2}$ aus (Dipp) $)_{2} \mathrm{NacNacGaI}_{2}$ und Superhydrid}

$\mathrm{Zu} 1.00 \mathrm{~g}(1.35 \mathrm{mmol})(\mathrm{Dipp})_{2} \mathrm{NacNacGaI}_{2}$ gelöst in $50 \mathrm{ml}$ Benzol werden 2 Äquivalente $(2.7 \mathrm{ml}, 2.70 \mathrm{mmol}) 1 \mathrm{M}$ Superhydridlösung (in THF gelöst) getropft. Die Lösung wird $12 \mathrm{~h}$ gerührt. Dabei entsteht LiI, das vollständig gelöst bleibt. Es werden alle flüchtigen Bestandteile im Vakuum destillativ entfernt. Der feste Rückstand wird in $n$-Hexan aufgenommen, wobei sich das Produkt löst und das LiI nahezu ungelöst bleibt. Die Suspension wird filtriert und das $n$-Hexan wird im Vakuum abdestilliert. Das Produkt kristallisiert in Diethylether bei $-27^{\circ} \mathrm{C}$ mit einer Ausbeute von $75 \%$ (0.49 g, $1.0 \mathrm{mmol})$. Das Produkt ist mit ca. $5 \%$ LiI verunreinigt, da sich LiI zu einem geringen Anteil in $n$-Hexan löst.

Schmelzpunkt: $135^{\circ} \mathrm{C}$.

${ }^{1}$ H-NMR $\left(300.13 \mathrm{MHz}, \mathrm{CDCl}_{3}\right): \delta 1.19\left(\mathrm{~d}, J=6.8 \mathrm{~Hz}, 12 \mathrm{H}, \mathrm{CH}\left(\mathrm{CH}_{3}\right)_{2}, 1.23(\mathrm{~d}\right.$, $J=6.8 \mathrm{~Hz}, 12 \mathrm{H}, \mathrm{CH}\left(\mathrm{CH}_{3}\right)_{2}, 1.72\left(\mathrm{~s}, 6 \mathrm{H}, \mathrm{CH}_{3}\right), 3.20$ (sept, J=6.8 Hz, $4 \mathrm{H}$, $\mathrm{CH}\left(\mathrm{CH}_{3}\right)_{2}, 4.58$ (br s, $\left.2 \mathrm{H}, \mathrm{Ga} H\right), 4.85(\mathrm{~s}, 1 \mathrm{H}, \mathrm{CH}), 7.10-7.24(\mathrm{~m}, 6 \mathrm{H}, \mathrm{ArH}) \mathrm{ppm}$.

${ }^{13}$ C-NMR $\left(125.75 \mathrm{MHz}, \mathrm{C}_{6} \mathrm{D}_{6}\right): \delta 22.93\left(\mathrm{NC}\left(\mathrm{CH}_{3}\right)\right), 24.46\left(\mathrm{CH}\left(\mathrm{CH}_{3}\right)_{2}\right), 25.52$ $\left(\mathrm{CH}\left(\mathrm{CH}_{3}\right)_{2}\right), 28.30\left(\mathrm{CH}\left(\mathrm{CH}_{3}\right)_{2}\right), 94.43(\gamma-C), 124.64(\mathrm{ArC}), 127.14(\mathrm{ArC}), 141.09$ $(\mathrm{ArC}), 144.11(\mathrm{ArC}), 168.13(\mathrm{NC}) \mathrm{ppm}$.

MS: m/e (\%) $488(\mathrm{M}, 65), 487(\mathrm{M}-\mathrm{H}, 100), 486(\mathrm{M}-2 \mathrm{H}, 8)$. 
IR (KBr, Nujol) $\tilde{v}$ : 1893 (st), 1861 (st), 1562 (m), 1526 (st), 1405 (s), 1320 (m), 1263 (s), 1253 (s), 1233 (s), 1176 (m), 1101 (m), 1055 (s), 1023 (m), 965 (s), 935 (m), 869 (s), 799 (st), 763 (st), 743 (st), 722 (m), 713 (m), 666 (m), 638 (m), 596 (s), $545(\mathrm{~s}), 526(\mathrm{~s}), 500(\mathrm{~m}), 441(\mathrm{~s})$.

Elementaranalyse: ber.: C 71.17, H 8.86, Ga 14.25, N 5.72

gef.: C 66.36, H 8.43, Ga 11.39, N 5.15, I 6.76.

\subsection{3.b Darstellung von (Dipp) $)_{2} \mathrm{NacNacGaH}_{2}$ aus (Dipp) $)_{2} \mathrm{NacNacGaCl}_{2}$ und Superhydrid}

$\mathrm{Zu} 1.00 \mathrm{~g}$ (1.79 mmol) (Dipp) ${ }_{2} \mathrm{NacNacGaCl}_{2}$ gelöst in $50 \mathrm{ml}$ Benzol werden 2 Äquivalente (3.6 ml, $3.58 \mathrm{mmol}) 1 \mathrm{M}$ Superhydridlösung (in THF gelöst) getropft. Die Lösung wird $12 \mathrm{~h}$ lang gerührt. Dabei entsteht LiCl. Es werden alle flüchtigen Bestandteile im Vakuum destillativ entfernt. Der feste Rückstand wird in $n$-Hexan aufgenommen, wobei sich das Produkt löst und das $\mathrm{LiCl}$ ungelöst bleibt. Die Suspension wird filtriert und das $n$-Hexan wird im Vakuum abdestilliert. Das Produkt kristallisiert in Diethylether bei $-27^{\circ} \mathrm{C}$ mit einer Ausbeute von $80 \%(0.70 \mathrm{~g}$, $1.4 \mathrm{mmol})$.

Schmelzpunkt: $135^{\circ} \mathrm{C}$. 
${ }^{1}$ H-NMR $\left(300.13 \mathrm{MHz}, \mathrm{CDCl}_{3}\right): \delta 1.19\left(\mathrm{~d}, J=6.8 \mathrm{~Hz}, 12 \mathrm{H}, \mathrm{CH}\left(\mathrm{CH}_{3}\right)_{2}, 1.23(\mathrm{~d}\right.$, $J=6.8 \mathrm{~Hz}, 12 \mathrm{H}, \mathrm{CH}\left(\mathrm{CH}_{3}\right)_{2}, 1.72\left(\mathrm{~s}, 6 \mathrm{H}, \mathrm{CH}_{3}\right), 3.20$ (sept, J=6.8 Hz, $4 \mathrm{H}$, $\mathrm{CH}\left(\mathrm{CH}_{3}\right)_{2}, 4.58$ (br s, $\left.2 \mathrm{H}, \mathrm{GaH}\right), 4.85$ (s, $\left.1 \mathrm{H}, \mathrm{CH}\right), 7.10-7.24$ (m, $\left.6 \mathrm{H}, \mathrm{ArH}\right) \mathrm{ppm}$.

${ }^{13}$ C-NMR $\left(125.75 \mathrm{MHz}, \mathrm{C}_{6} \mathrm{D}_{6}\right): \delta 22.93\left(\mathrm{NC}\left(\mathrm{CH}_{3}\right)\right), 24.46\left(\mathrm{CH}\left(\mathrm{CH}_{3}\right)_{2}\right), 25.52$ $\left(\mathrm{CH}\left(\mathrm{CH}_{3}\right)_{2}\right), 28.30\left(\mathrm{CH}\left(\mathrm{CH}_{3}\right)_{2}\right), 94.43(\gamma-\mathrm{C}), 124.64(\mathrm{ArC}), 127.14(\mathrm{ArC}), 141.09$ $(\mathrm{ArC}), 144.11(\mathrm{ArC}), 168.13(\mathrm{NC}) \mathrm{ppm}$.

MS: m/e (\%) $488(\mathrm{M}, 65), 487(\mathrm{M}-\mathrm{H}, 100), 486(\mathrm{M}-2 \mathrm{H}, 8)$.

IR (KBr, Nujol) $\tilde{v}$ : 1893 (st), 1861 (st), 1562 (m), 1526 (st), 1405 (s), 1320 (m), 1263 (s), 1253 (s), 1233 (s), 1176 (m), 1101 (m), 1055 (s), 1023 (m), 965 (s), 935 (m), 869 (s), 799 (st), 763 (st), 743 (st), 722 (m), 713 (m), 666 (m), 638 (m), 596 (s), $545(\mathrm{~s}), 526(\mathrm{~s}), 500(\mathrm{~m}), 441(\mathrm{~s})$.

Elementaranalyse: ber.: C 71.17, H 8.86, Ga 14.25, N 5.72 gef.: C 71.25, H 8.89, Ga 14.19, N 5.73

\subsubsection{Darstellung von (Dipp) ${ }_{2} \mathrm{NacNacGaHCl}$}

$\mathrm{Zu} 1.00 \mathrm{~g}$ (2.05 mmol) $\mathrm{NacNacGaH}_{2}$ gelöst in $50 \mathrm{ml}$ Benzol wird 1 Äquivalent (0.22 g, $2.05 \mathrm{mmol})$ TMSCl gelöst in $20 \mathrm{ml}$ Benzol getropft. Die Lösung wird $24 \mathrm{~h}$ lang gerührt. Danach werden alle flüchtigen Bestandteile im Vakuum destillativ entfernt. Der feste Rückstand wird in $30 \mathrm{ml}$ Diethylether aufgenommen und filtriert. 
Bei der erhaltenen Substanz handelt es sich um ein Gemisch aus (Dipp) ${ }_{2} \mathrm{NacNacGaH}_{2}$, (Dipp) ${ }_{2} \mathrm{NacNacGaHCl}$ und (Dipp) ${ }_{2} \mathrm{NacNacGaCl}_{2}$. Spektroskopische Daten von (Dipp) ${ }_{2} \mathrm{NacNacGaHCl:}$

${ }^{1}$ H-NMR $\left(500.13 \mathrm{MHz}, \mathrm{CDCl}_{3}\right): \delta 1.08\left(\mathrm{~d}, J=6.8 \mathrm{~Hz}, 12 \mathrm{H}, \mathrm{CH}\left(\mathrm{CH}_{3}\right)_{2}, 1.15(\mathrm{~d}\right.$, $J=6.8 \mathrm{~Hz}, 12 \mathrm{H}, \mathrm{CH}\left(\mathrm{CH}_{3}\right)_{2}, 1.53\left(\mathrm{~s}, 6 \mathrm{H}, \mathrm{CH}_{3}\right), 3.62$ (sept, J=6.8 Hz, $4 \mathrm{H}$, $\mathrm{CH}\left(\mathrm{CH}_{3}\right)_{2}, 5.45$ (br s, $\left.1 \mathrm{H}, \mathrm{GaH}\right), 4.79(\mathrm{~s}, 1 \mathrm{H}, \mathrm{CH}), 6.90-7.20(\mathrm{~m}, 6 \mathrm{H}, \mathrm{ArH}) \mathrm{ppm}$.

MS: m/e (\%) 523 (M, 40), $471(\mathrm{M}-\mathrm{H}-\mathrm{Cl}-\mathrm{Me}, 100)$.

\subsection{Untersuchung der Reaktivität von (Dipp) ${ }_{2} \mathrm{NacNacGaH}_{2}$ gegenüber unterschiedlichen Funktionalitäten}

\subsubsection{Umsetzung von (Dipp) ${ }_{2} \mathrm{NacNacGaH}_{2}$ mit $\mathrm{H}-\mathrm{C} \equiv \mathrm{C}-\mathrm{Ph}$}

$\mathrm{Zu} \quad 1.00 \mathrm{~g} \quad(2.04 \mathrm{mmol}) \quad(\mathrm{Dipp})_{2} \mathrm{NacNacGaH}_{2}$ gelöst in $50 \mathrm{ml}$ Xylol werden 9 Äquivalente ( 1.88 g, 18.4 mmol) Phenylacetylen getropft. Die Reaktionsmischung wird 7 Tage lang am Rückfluß erhitzt. Nach dem Abkühlen werden alle flüchtigen Bestandteile destillativ entfernt. Der erhaltene Feststoff wird in $50 \mathrm{ml}$ Diethylether aufgenommen. Unlösliche Substanzen werden durch Filtration abgetrennt. Nach dem Abdestillieren des Diethylethers wird ausschließlich (Dipp) ${ }_{2} \mathrm{NacNacH}$ erhalten. 
${ }^{1}$ H-NMR $\left(500.13 \mathrm{MHz}, \mathrm{C}_{6} \mathrm{D}_{6}\right): \delta 1.13\left(\mathrm{~d}, J=6.8 \mathrm{~Hz}, 12 \mathrm{H}, \mathrm{CH}\left(\mathrm{CH}_{3}\right)_{2}, 1.19(\mathrm{~d}\right.$, $J=6.8 \mathrm{~Hz}, 12 \mathrm{H}, \mathrm{CH}\left(\mathrm{CH}_{3}\right)_{2}, 1.63\left(\mathrm{~s}, 6 \mathrm{H}, \mathrm{CH}_{3}\right), 3.28$ (sept, J=6.8 Hz, $4 \mathrm{H}$, $\mathrm{CH}\left(\mathrm{CH}_{3}\right)_{2}, 4.86(\mathrm{~s}, 1 \mathrm{H}, \mathrm{CH}), 7.10-7.16(\mathrm{~m}, 6 \mathrm{H}, \mathrm{Ar} H), 12.40$ (br s, $\left.1 \mathrm{H}, \mathrm{NH}\right) \mathrm{ppm}$.

\subsubsection{Umsetzung von (Dipp) ${ }_{2} \mathrm{NacNacGaH}_{2}$ mit 1,2-cis-Diphenylethen}

$\mathrm{Zu} \quad 1.00 \mathrm{~g} \quad(2.04 \mathrm{mmol}) \quad(\mathrm{Dipp})_{2} \mathrm{NacNacGaH}_{2}$ gelöst in $50 \mathrm{ml}$ Benzol werden 9 Äquivalente $\quad(3.24 \mathrm{~g}, \quad 18.4 \mathrm{mmol}) \quad$ 1,2-cis-Diphenylethen getropft. Die Reaktionsmischung wird 24 h lang am Rückfluß erhitzt. Nach dem Abkühlen werden alle flüchtigen Bestandteile destillativ entfernt. Der erhaltene Feststoff wird in $50 \mathrm{ml}$ Diethylether aufgenommen. Unlösliche Substanzen werden durch Filtration abgetrennt. Nach dem Abdestillieren des Diethylethers werden $0.92 \mathrm{~g}(1.8 \mathrm{mmol})$ (Dipp) ${ }_{2} \mathrm{NacNacGaH}_{2}$ erhalten.

${ }^{\mathbf{1}} \mathbf{H}-\mathbf{N M R}\left(300.13 \mathrm{MHz}, \mathrm{CDCl}_{3}\right): \delta 1.19\left(\mathrm{~d}, J=6.8 \mathrm{~Hz}, 12 \mathrm{H}, \mathrm{CH}\left(\mathrm{CH}_{3}\right)_{2}, 1.23(\mathrm{~d}\right.$, $J=6.8 \mathrm{~Hz}, 12 \mathrm{H}, \mathrm{CH}\left(\mathrm{CH}_{3}\right)_{2}, 1.72\left(\mathrm{~s}, 6 \mathrm{H}, \mathrm{CH}_{3}\right), 3.20$ (sept, J=6.8 Hz, $4 \mathrm{H}$, $\mathrm{CH}\left(\mathrm{CH}_{3}\right)_{2}, 4.58$ (br s, $\left.2 \mathrm{H}, \mathrm{Ga} H\right), 4.85(\mathrm{~s}, 1 \mathrm{H}, \mathrm{CH}), 7.10-7.24$ (m, $\left.6 \mathrm{H}, \mathrm{ArH}\right) \mathrm{ppm}$.

\subsubsection{Umsetzung von (Dipp) ${ }_{2} \mathrm{NacNacGaH}_{2}$ mit ICN}

$\mathrm{Zu} 1.00 \mathrm{~g}(2.04 \mathrm{mmol})(\mathrm{Dipp})_{2} \mathrm{NacNacGaH}_{2}$ gelöst in $40 \mathrm{ml}$ Benzol wird ein Äquivalent (0.312 g, $2.04 \mathrm{mmol})$ ICN gelöst in $40 \mathrm{ml}$ Benzol getropft. Die Lösung wird $24 \mathrm{~h}$ lang gerührt. Bei der Reaktion entsteht ein unlöslicher Feststoff der abfiltriert wird. Danach werden alle flüchtigen Bestandteile im Vakuum destillativ 
entfernt. Der feste Rückstand wird in $30 \mathrm{ml}$ Diethylether aufgenommen, filtriert und kristallisiert bei $-27^{\circ} \mathrm{C}$. Die erhaltenen Einkristalle wurden röngenographisch untersucht. Bei dem entstandenem Produkt handelt es sich um das literaturbekannte (Dipp) ${ }_{2} \mathrm{NacNacGaI}_{2}$.

${ }^{1}$ H-NMR $\left(300.13 \mathrm{MHz}, \mathrm{C}_{6} \mathrm{D}_{6}\right): \delta 1.15\left(\mathrm{~d}, J=6.8 \mathrm{~Hz}, 12 \mathrm{H}, \mathrm{CH}\left(\mathrm{CH}_{3}\right)_{2}, 1.20(\mathrm{~d}\right.$, $J=6.8 \mathrm{~Hz}, 12 \mathrm{H}, \mathrm{CH}\left(\mathrm{CH}_{3}\right)_{2}, 1.66\left(\mathrm{~s}, 6 \mathrm{H}, \mathrm{CH}_{3}\right), 3.30$ (sept, J=6.8 Hz, $4 \mathrm{H}$, $\mathrm{CH}\left(\mathrm{CH}_{3}\right)_{2}, 4.85(\mathrm{~s}, 1 \mathrm{H}, \mathrm{CH}), 6.90-7.20(\mathrm{~m}, 6 \mathrm{H}, \mathrm{Ar} H) \mathrm{ppm}$.

\subsubsection{Umsetzung von (Dipp) ${ }_{2} \mathrm{NacNacGaH}_{2}$ mit Benzonitril}

$1.00 \mathrm{~g}$ (2.04 mmol) (Dipp) ${ }_{2} \mathrm{NacNacGaH}_{2}$ werden in $15 \mathrm{ml}$ Benzonitril gelöst und $24 \mathrm{~h}$ lang auf $180^{\circ} \mathrm{C}$ erhitzt. Nach dem Abkühlen wird das Benzonitril im Vakuum entfernt. Der erhaltene Feststoff wird in $50 \mathrm{ml}$ Diethylether aufgenommen. Unlösliche Substanzen werden durch Filtration abgetrennt. Nach dem Abdestillieren des Diethylethers werden $0.82 \mathrm{~g}(1.67 \mathrm{mmol})(\mathrm{Dipp})_{2} \mathrm{NacNacH}$ erhalten.

${ }^{1}$ H-NMR $\left(500.13 \mathrm{MHz}, \mathrm{C}_{6} \mathrm{D}_{6}\right): \delta 1.13\left(\mathrm{~d}, J=6.8 \mathrm{~Hz}, 12 \mathrm{H}, \mathrm{CH}\left(\mathrm{CH}_{3}\right)_{2}, 1.19(\mathrm{~d}\right.$, $J=6.8 \mathrm{~Hz}, 12 \mathrm{H}, \mathrm{CH}\left(\mathrm{CH}_{3}\right)_{2}, 1.63\left(\mathrm{~s}, 6 \mathrm{H}, \mathrm{CH}_{3}\right), 3.28$ (sept, J=6.8 Hz, $4 \mathrm{H}$, $\mathrm{CH}\left(\mathrm{CH}_{3}\right)_{2}, 4.86(\mathrm{~s}, 1 \mathrm{H}, \mathrm{CH}), 7.10-7.16(\mathrm{~m}, 6 \mathrm{H}, \mathrm{Ar} H), 12.40$ (br s, $\left.1 \mathrm{H}, \mathrm{NH}\right) \mathrm{ppm}$. 


\subsubsection{Umsetzung von (Dipp) ${ }_{2} \mathrm{NacNacGaH}_{2}$ mit Methylhydrazin}

$\mathrm{Zu} \quad 1.00 \mathrm{~g} \quad(2.04 \mathrm{mmol}) \quad(\mathrm{Dipp})_{2} \mathrm{NacNacGaH}_{2}$ gelöst in $50 \mathrm{ml}$ Benzol werden 9 Äquivalente $(0.84$ g, 18 mmol) Methylhydrazin getropft. Die Reaktionsmischung wird $24 \mathrm{~h}$ lang am Rückfluß erhitzt. Nach dem Abkühlen werden alle flüchtigen Bestandteile destillativ entfernt. Der erhaltene Feststoff wird in $50 \mathrm{ml}$ Diethylether aufgenommen. Unlösliche Substanzen werden durch Filtration abgetrennt. Nach dem Abdestillieren des Diethylethers werden $0.96 \mathrm{~g}$ (1.9 mmol) (Dipp) ${ }_{2} \mathrm{NacNacGaH}_{2}$ erhalten.

${ }^{1}$ H-NMR $\left(300.13 \mathrm{MHz}, \mathrm{CDCl}_{3}\right): \delta 1.19\left(\mathrm{~d}, J=6.8 \mathrm{~Hz}, 12 \mathrm{H}, \mathrm{CH}\left(\mathrm{CH}_{3}\right)_{2}, 1.23(\mathrm{~d}\right.$, $J=6.8 \mathrm{~Hz}, 12 \mathrm{H}, \mathrm{CH}\left(\mathrm{CH}_{3}\right)_{2}, 1.72\left(\mathrm{~s}, 6 \mathrm{H}, \mathrm{CH}_{3}\right), 3.20$ (sept, J=6.8 Hz, $4 \mathrm{H}$, $\mathrm{CH}\left(\mathrm{CH}_{3}\right)_{2}, 4.58$ (br s, $\left.2 \mathrm{H}, \mathrm{Ga} H\right), 4.85$ (s, $\left.1 \mathrm{H}, \mathrm{CH}\right), 7.10-7.24$ (m, $\left.6 \mathrm{H}, \mathrm{ArH}\right) \mathrm{ppm}$.

\subsubsection{Umsetzung von (Dipp) ${ }_{2} \mathrm{NacNacGaH}_{2}$ mit tert-Butylhydroperoxid}

$\mathrm{Zu} \quad 1.00 \mathrm{~g} \quad(2.04 \mathrm{mmol}) \quad(\mathrm{Dipp})_{2} \mathrm{NacNacGaH}_{2}$ gelöst in $50 \mathrm{ml}$ Benzol werden 9 Äquivalente $\quad(1.62 \mathrm{~g}, \quad 18.4 \mathrm{mmol}) \quad$ tert-Butylhydroperoxid getropft. Die Reaktionsmischung wird $24 \mathrm{~h}$ lang am Rückfluß erhitzt. Nach dem Abkühlen werden alle flüchtigen Bestandteile destillativ entfernt. Der erhaltene Feststoff wird in $50 \mathrm{ml}$ Diethylether aufgenommen. Unlösliche Substanzen werden durch Filtration abgetrennt. Nach dem Abdestillieren des Diethylethers werden $0.72 \mathrm{~g}(1.5 \mathrm{mmol})$ (Dipp) ${ }_{2} \mathrm{NacNacGaH}_{2}$ erhalten. 
${ }^{1}$ H-NMR $\left(300.13 \mathrm{MHz}, \mathrm{CDCl}_{3}\right): \delta 1.19\left(\mathrm{~d}, J=6.8 \mathrm{~Hz}, 12 \mathrm{H}, \mathrm{CH}\left(\mathrm{CH}_{3}\right)_{2}, 1.23(\mathrm{~d}\right.$, $J=6.8 \mathrm{~Hz}, 12 \mathrm{H}, \mathrm{CH}\left(\mathrm{CH}_{3}\right)_{2}, 1.72\left(\mathrm{~s}, 6 \mathrm{H}, \mathrm{CH}_{3}\right), 3.20$ (sept, J=6.8 Hz, $4 \mathrm{H}$, $\mathrm{CH}\left(\mathrm{CH}_{3}\right)_{2}, 4.58$ (br s, $\left.2 \mathrm{H}, \mathrm{GaH}\right), 4.85(\mathrm{~s}, 1 \mathrm{H}, \mathrm{CH}), 7.10-7.24(\mathrm{~m}, 6 \mathrm{H}, \mathrm{ArH}) \mathrm{ppm}$.

\subsubsection{Umsetzung von (Dipp) ${ }_{2} \mathrm{NacNacGaH}_{2}$ mit Trifluorethanol}

$\mathrm{Zu} \quad 1.00 \mathrm{~g} \quad(2.04 \mathrm{mmol}) \quad(\mathrm{Dipp})_{2} \mathrm{NacNacGaH}_{2}$ gelöst in $50 \mathrm{ml}$ Benzol werden 9 Äquivalente (1.84 g, $18.4 \mathrm{mmol})$ Trifluorethanol getropft. Die Reaktionsmischung wird $24 \mathrm{~h}$ lang am Rückfluß erhitzt. Nach dem Abkühlen werden alle flüchtigen Bestandteile destillativ entfernt. Der erhaltene Feststoff wird in $50 \mathrm{ml}$ Diethylether aufgenommen. Unlösliche Substanzen werden durch Filtration abgetrennt. Nach dem Abdestillieren des Diethylethers werden $0.89 \mathrm{~g}$ (1.8 mmol) (Dipp) ${ }_{2} \mathrm{NacNacGaH}_{2}$ erhalten.

${ }^{1}$ H-NMR $\left(300.13 \mathrm{MHz}, \mathrm{CDCl}_{3}\right): \delta 1.19\left(\mathrm{~d}, J=6.8 \mathrm{~Hz}, 12 \mathrm{H}, \mathrm{CH}\left(\mathrm{CH}_{3}\right)_{2}, 1.23(\mathrm{~d}\right.$, $J=6.8 \mathrm{~Hz}, 12 \mathrm{H}, \mathrm{CH}\left(\mathrm{CH}_{3}\right)_{2}, 1.72\left(\mathrm{~s}, 6 \mathrm{H}, \mathrm{CH}_{3}\right), 3.20$ (sept, J=6.8 Hz, $4 \mathrm{H}$, $\mathrm{CH}\left(\mathrm{CH}_{3}\right)_{2}, 4.58$ (br s, $\left.2 \mathrm{H}, \mathrm{Ga} H\right), 4.85(\mathrm{~s}, 1 \mathrm{H}, \mathrm{CH}), 7.10-7.24$ (m, $\left.6 \mathrm{H}, \mathrm{ArH}\right) \mathrm{ppm}$.

\subsection{Umsetzung von (Dipp) $)_{2} \mathrm{NacNacGaH}_{2}$ mit Säuren}

\subsubsection{Umsetzung von (Dipp) ${ }_{2} \mathrm{NacNacGaH}_{2}$ mit Benzoesäure}

$\mathrm{Zu} 1.00 \mathrm{~g}$ (2.04 mmol) (Dipp) ${ }_{2} \mathrm{NacNacGaH}_{2}$ gelöst in $50 \mathrm{ml}$-Xylol werden 2 Äquivalente $(0.50 \mathrm{~g}, 4.08 \mathrm{mmol})$ Benzoesäure gelöst in $30 \mathrm{ml} p$-Xylol getropft. Die 
Lösung wird 24 h lang am Rückfluss erhitzt. Es bildet sich eine unlösliche Substanz, die nach dem Abkühlen auf Raumtemperatur abfiltriert wird. Von der klaren Lösung werden alle flüchtigen Bestandteile im Vakuum destillativ entfernt. Der feste Rückstand wird in $30 \mathrm{ml}$ Diethylether aufgenommen und filtriert. Das Produkt kristallisiert in Diethylether bei $-27^{\circ} \mathrm{C}$ mit einer Ausbeute von $44 \%(0.60 \mathrm{~g}$, $0.9 \mathrm{mmol}$ ). Dabei handelt es sich nicht um die Zielverbindung, sondern um $\left[(\mathrm{Dipp})_{2} \mathrm{NacNacH}_{2}\right]^{+}[\mathrm{OOCPh}]^{-}$. Bei der massenspektrometrischen Untersuchung der unlöslichen Komponente lässt sich (Dipp) ${ }_{2} \mathrm{NacNacGa}(\mathrm{OOCPh})_{2}$ nachweisen.

Schmelzpunkt: $184{ }^{\circ} \mathrm{C}$.

Analytischer Befund der löslichen Komponente:

${ }^{1}$ H-NMR $\left(300.13 \mathrm{MHz}, \mathrm{C}_{6} \mathrm{D}_{6}\right): \delta 1.15\left(\mathrm{~d}, J=6.8 \mathrm{~Hz}, 12 \mathrm{H}, \mathrm{CH}\left(\mathrm{CH}_{3}\right)_{2}, 1.21(\mathrm{~d}\right.$, $J=6.8 \mathrm{~Hz}, 12 \mathrm{H}, \mathrm{CH}\left(\mathrm{CH}_{3}\right)_{2}, 1.66\left(\mathrm{~s}, 6 \mathrm{H}, \mathrm{CH}_{3}\right), 2,13$ (s, $\left.2 \mathrm{H}, \mathrm{NH}\right), 3.31$ (sept, $J=6.8 \mathrm{~Hz}, 4 \mathrm{H}, \mathrm{CH}\left(\mathrm{CH}_{3}\right)_{2}, 4.87(\mathrm{~s}, 1 \mathrm{H}, \mathrm{CH}), 6.90-7.20(\mathrm{~m}, 16 \mathrm{H}, \mathrm{ArH}) \mathrm{ppm}$.

Analytischer Befund der unlöslichen Komponente:

MS: m/e (\%) 728 (M, 3), 607 (M - Benzoat, 6), 507 (M - Benzoat - 2 iso-Propyl Me, 100).

\subsubsection{Umsetzung von (Dipp) ${ }_{2} \mathrm{NacNacGaH}_{2}$ mit Essigsäure}

$\mathrm{Zu} 1.00 \mathrm{~g}$ (2.04 mmol) (Dipp) ${ }_{2} \mathrm{NacNacGaH}_{2}$ gelöst in $50 \mathrm{ml}$ Benzol werden 2 Äquivalente (0.245 g, 4.08 mmol) Essigsäure getropft. Die Lösung wird 12 h lang am 
Rückfluß gerührt. Danach werden alle flüchtigen Bestandteile im Vakuum destillativ entfernt. Der feste Rückstand wird in $30 \mathrm{ml}$ Diethylether aufgenommen und filtriert. Die erhaltene Substanz kristallisiert in Diethylether bei $-27{ }^{\circ} \mathrm{C}$ mit einer Ausbeute von $18 \% \quad(0.20 \mathrm{~g}, \quad 0.37 \mathrm{mmol})$. Dabei handelt es sich um $\left[(\text { Dipp })_{2} \mathrm{NacNaCH}_{3}\right]^{2+}\left[\mathrm{OOCCH}_{3}\right]_{2}^{-}$. Bei der massenspektrometrischen Untersuchung der unlöslichen Komponente lässt sich (Dipp) ${ }_{2} \mathrm{NacNacGa}\left(\mathrm{OOCCH}_{3}\right)_{2}$ nachweisen.

Schmelzpunkt: $160^{\circ} \mathrm{C}$.

Analytischer Befund der löslichen Komponente:

${ }^{1}$ H-NMR $\left(300.13 \mathrm{MHz}, \mathrm{CDCl}_{3}\right): \delta 1.10\left(\mathrm{~d}, J=6.8 \mathrm{~Hz}, 12 \mathrm{H}, \mathrm{CH}\left(\mathrm{CH}_{3}\right)_{2}, 1.19(\mathrm{~d}\right.$, $J=6.8 \mathrm{~Hz}, 12 \mathrm{H}, \mathrm{CH}\left(\mathrm{CH}_{3}\right)_{2}, 1.70\left(\mathrm{~s}, 6 \mathrm{H}, \mathrm{CH}_{3}\right), 2.08$ (s, $\left.6 \mathrm{H}, \mathrm{O}=\mathrm{CCH}_{3}\right), 3.09$ (sept, $J=6.8 \mathrm{~Hz}, 4 \mathrm{H}, \mathrm{CH}\left(\mathrm{CH}_{3}\right)_{2}, 4.85(\mathrm{~s}, 1 \mathrm{H}, \mathrm{CH}), 7.09-7.34(\mathrm{~m}, 6 \mathrm{H}, \mathrm{ArH}), 9.50-$ 11.18 (br s, $2 \mathrm{H}, \mathrm{NH}_{2}$ ) ppm.

${ }^{13}$ C-NMR $\left(75.47 \mathrm{MHz}, \mathrm{CDCl}_{3}\right): \quad \delta 15.26\left(\mathrm{O}=\mathrm{CCH}_{3}\right), 20.74\left(\mathrm{NC}\left(\mathrm{CH}_{3}\right)\right), 23.21$ $\left(\mathrm{CH}\left(\mathrm{CH}_{3}\right)_{2}\right), 254.26\left(\mathrm{CH}\left(\mathrm{CH}_{3}\right)_{2}\right), 28.19\left(\mathrm{CH}_{\left.\left(\mathrm{CH}_{3}\right)_{2}\right),}, 93.23(\gamma-\mathrm{C}), 123.01(\mathrm{ArC})\right.$, $123.20(\mathrm{ArC}), 125.07(\mathrm{ArC}), 128.33(\mathrm{ArC}), 140.73(\mathrm{NC}), 142.46(\mathrm{NC}), 161.18$ $\left(\mathrm{O}=\mathrm{CCH}_{3}\right) \mathrm{ppm}$.

Analytischer Befund der unlöslichen Komponente:

MS: m/e (\%) 604 (M, 1), 418 ((Dipp) $\left.{ }_{2} \mathrm{NacNac}, 30\right), 403$ ((Dipp) $\left.{ }_{2} \mathrm{NacNac}-\mathrm{Me}, 90\right)$, 187 (Dipp-N-C, 100). 


\subsubsection{Umsetzung von (Dipp) ${ }_{2} \mathrm{NacNacGaH}_{2}$ mit Trifluoressigsäure}

$\mathrm{Zu} 1.00 \mathrm{~g}$ (2.04 mmol) (Dipp) ${ }_{2} \mathrm{NacNacGaH}_{2}$ gelöst in $50 \mathrm{ml}$ Benzol werden 2 Äquivalente $(0.46 \mathrm{~g}, 4.08 \mathrm{~mol})$ Trifluoressigsäure getropft. Die Lösung wird $12 \mathrm{~h}$ lang bei Raumtemperatur gerührt. Es werden alle flüchtigen Bestandteile im Vakuum destillativ entfernt. Der feste Rückstand wird in $30 \mathrm{ml}$ Diethylether aufgenommen und filtriert. Das Produkt kristallisiert in Diethylether bei $-27^{\circ} \mathrm{C}$ mit einer Ausbeute von $90 \%(1.19 \mathrm{~g}, 1.84 \mathrm{mmol})$. Dabei handelt es sich nicht um die gewünschte Zielverbindung sondern um $\quad\left[(\mathrm{Dipp})_{2} \mathrm{NacNacH}_{2}\right]^{+}\left[\mathrm{OOCCF}_{3}\right]^{-}$. Bei der massenspektrometrischen Untersuchung der unlöslichen Komponente lässt sich (Dipp) ${ }_{2} \mathrm{NacNacGa}\left(\mathrm{OOCCF}_{3}\right)_{2}$ nachweisen.

Analytischer Befund der löslichen Komponente:

${ }^{1}$ H-NMR $\left(300.13 \mathrm{MHz}, \mathrm{CDCl}_{3}\right): \delta 0.81\left(\mathrm{~d}, J=6.8 \mathrm{~Hz}, 12 \mathrm{H}, \mathrm{CH}\left(\mathrm{CH}_{3}\right)_{2}, 0.10(\mathrm{~d}\right.$, $J=6.8 \mathrm{~Hz}, 12 \mathrm{H}, \mathrm{CH}\left(\mathrm{CH}_{3}\right)_{2}, 2.62\left(\mathrm{~s}, 6 \mathrm{H}, \mathrm{CH}_{3}\right), 3.56$ (sept, J=6.8 Hz, $4 \mathrm{H}$, $\mathrm{CH}\left(\mathrm{CH}_{3}\right)_{2}, 4.45(\mathrm{~s}, 1 \mathrm{H}, \mathrm{CH}), 6.90-7.40(\mathrm{~m}, 6 \mathrm{H}, \mathrm{ArH}), 10.03$ (br s, $2 \mathrm{H}, \mathrm{NH}_{2}$ ppm. ${ }^{19}$ F-NMR $\left(188.29 \mathrm{MHz}, \mathrm{CDCl}_{3}\right): \delta 85.64\left(\mathrm{~s}, \mathrm{CF}_{3}\right) \mathrm{ppm}$.

Analytischer Befund der unlöslichen Komponente:

MS: m/e (\%) $712(\mathrm{M}, 85), 599$ (M - Trifluoracetat, 28), 583 (M - Trifluoracetat Me, 100). 


\section{Ergebnisse}

Das Galliumhydridetherate thermisch instabil sind, ist bekannt. Frühere Arbeiten zeigen, dass Aluminiumhydridaminaddukte mit aciden Acetylenen und Nitrilen eine Vielzahl von Reaktionen eingehen, bei denen bei erhöhten Temperaturen und einem Unterschuss vom Hydrid, interessante Cluster gebildet werden. Vergleichbare Reaktionen mit Galliumhydridtrimetylamin waren nicht möglich, da sich dieses bei Temperaturen über $55{ }^{\circ} \mathrm{C}$ in $\mathrm{Ga}$ und $\mathrm{H}_{2}$ zersetzte. Im Rahmen dieses niedrigen Temperaturbereiches konnten allerdings einfache Galliumacetylidderivate dargestellt und charakterisiert werden. Weiterhin wurden Arduengocarbene als stabilisierende Basen eingesetzt. Im Falle des iso-Propylarduengocarbens konnten nur geringe Ausbeuten erzielt werden. Mesitylarduengocarbengalliumhydrid ist sehr schlecht löslich und somit für weitere Reaktionen nur bedingt geeignet.

Mit (Dipp) $)_{2}$ NacNac als Substituent konnten die Fluor- und Bromderivate dargestellt und strukturell untersucht werden. Als weiteres vielversprechendes Hydrid konnte das (Dipp) ${ }_{2} \mathrm{NacNacGaH}_{2}$ hergestellt werden, was sich durch seine hohe thermische Stabilität und gute Löslichkeit auszeichnet. Der hydridische Charakter der Wasserstoffe ist allerdings zu gering um eine Derivatisierung mit Acetylenen oder Nitrilen zu ermöglichen. Bei Reaktionen mit stark aciden Reagenzien, wie organischen Säuren, wurde die Hydrolyse des (Dipp) ${ }_{2}$ NacNac-Substituenten beobachtet. Die Zielverbindungen sind unlöslich und konnten nur massenspektroskopisch nachgewiesen werden. Im Gegensatz $\mathrm{zu}$ den Galliumhydridaddukten konnte selektiv nur ein Hydridwasserstoff durch ein 
Halogenatom substituiert werden, was durch die Synthese von (Dipp) ${ }_{2} \mathrm{NacNacHCl}$ gezeigt wurde. 


\section{Behandlung und Entsorgung der Abfälle}

Die eingesetzten Lösungsmittel wurden abdestilliert oder im Vakuum in Kühlfallen einkondensiert und in die bereitgestellten Behälter gegeben.

Ethanol aus Kühlbädern wurde dem KOH-Reinigungsbad für Glasgeräte zugeführt. Natriumreste die bei Trocknungen von Lösungsmitteln anfielen, wurden mit iso-Propanol hydrolysiert und dem KOH-Reinigungsbad zugeführt.

Andere Trockenmittelreste wie Calciumhydrid oder $\mathrm{P}_{4} \mathrm{O}_{10}$ wurden nach vollständiger Hydrolyse mit Wasser in die Basen- bzw. Säureabfälle gegeben.

Konzentrierte Salpetersäure und Königswasser zur Reinigung von Glasfiltern wurden in den Säureabfallbehältern gesammelt.

Schwermetallfreie Chemiekalienrückstände wurden hydrolysiert und in die Gefäße für saure bzw. basische Abfälle gegeben.

Die in die bereitgestellten Kanister entsorgten Abfälle wurden in ausliegenden Listen ordnungsgemäß deklariert.

Das KOH-Reinigungsbad wurde unter Spezifikation und Angabe des Gehalts aller Bestandteile am zentralen Sammellager der chemischen Institute zur Entsorgung abgegeben.

Das Säurebad wurde mit Natriumcarbonat neutralisiert und die entstandene Natriumchloridlösung dem kommunalen Abwassernetz zugeführt.

Die bei der Anfertigung dieser Arbeit angefallen Mengen Sonderabfall können wie folgt abgeschätzt werden:

Lösungsmittelabfälle $\quad 1501$

Säureabfälle

Basenabfälle 


\section{Röntgenographischer Anhang}

\subsection{Verbindung}

Summenformel

Molare Masse

Temperatur

Wellenlänge

Kristallsystem

Raumgruppe

Zelldimensionen

Zellvolumen

Formeleinheiten pro Zelle

Berechnete Dichte

Absorptionskoeffitient

F (000)

Kristallgröße

Gemessener $\theta$-Bereich

Indexbereich

Anzahl der gemessenen Reflexe

Unabhängige Reflexe

Vollständigkeit an $\theta=22.54^{\circ}$

Max. und min. Transmission

Verfeinerungsmethode

Daten / Restraints / Parameter

Goodness-of-Fit an $\mathrm{F}^{2}$

Endgültige R-Werte [I > $2 \sigma$ (I)]

R-Werte (sämtliche Daten)

Größtes Maximum und Minimum

\author{
$\mathrm{Me}_{3} \mathbf{N} \cdot \mathrm{Ga}(\mathrm{C} \equiv \mathrm{C}-\mathrm{Ph})_{3}$ \\ $\mathrm{C}_{27} \mathrm{H}_{24} \mathrm{GaN}$ \\ 432.19 \\ 203(2) K \\ $71.073 \mathrm{pm}$ \\ monoklin \\ $\mathrm{P} 2(1) / \mathrm{n}$ \\ $\mathrm{a}=1391.9(2) \mathrm{pm}$ \\ $\alpha=90^{\circ}$ \\ $\mathrm{b}=1192.12(15) \mathrm{pm}$ \\ $\beta=94.363(16)^{\circ}$ \\ $\mathrm{c}=1401.9(4) \mathrm{pm}$ \\ $\gamma=90^{\circ}$
}

4

$1.238 \mathrm{Mg} / \mathrm{m}^{3}$

$1.198 \mathrm{~mm}^{-1}$

896

$0.80 \times 0.80 \times 0.40 \mathrm{~mm}^{3}$

$3.59-22.54^{\circ}$

$-15<=\mathrm{h}<=15,-8<=\mathrm{k}<=12,-12<=\mathrm{l}<=15$

3241

$3022[\mathrm{R}(\mathrm{int})=0.1175]$

$99.4 \%$

0.6459 und 0.4475

Full-matrix least-squares on $\mathrm{F}^{2}$

3022 / 0 / 265

1.184

$\mathrm{R} 1=0.0435, \mathrm{wR} 2=0.1212$

$\mathrm{R} 1=0.0455, \mathrm{wR} 2=0.1231$

0.827 und -0.706 e. $\AA^{-3}$ 


\subsection{Verbindung}

Summenformel

Molare Masse

Temperatur

Wellenlänge

Kristallsystem

Raumgruppe

Zelldimensionen

Zellvolumen

Formeleinheiten pro Zelle

Berechnete Dichte

Absorptionskoeffitient

F (000)

Kristallgröße

Gemessener $\theta$-Bereich

Indexbereich

Anzahl der gemessenen Reflexe

Unabhängige Reflexe

Vollständigkeit an $\theta=22.54^{\circ}$

Max. und min. Transmission

Verfeinerungsmethode

Daten / Restraints / Parameter

Goodness-of-Fit an $\mathrm{F}^{2}$

Endgültige R-Werte [I $>2 \sigma(\mathrm{I})]$

R-Werte (sämtliche Daten)

Größtes Maximum und Minimum

\section{$\mathrm{Me}_{3} \mathrm{~N} \cdot \mathrm{Ga}(\mathrm{C} \equiv \mathrm{C}-\mathrm{TMS})_{2} \mathrm{Cl}$}

$\mathrm{C}_{13} \mathrm{H}_{27} \mathrm{ClGaNSi}_{2}$

358.71

133(2) K

$71.073 \mathrm{pm}$

triklin

P-1

$\mathrm{a}=660.96(6) \mathrm{pm}$

$\alpha=82.983(8)^{\circ}$

$\mathrm{b}=1052.18(10) \mathrm{pm}$

$\beta=82.129(8)^{\circ}$

$\mathrm{c}=1459.59(14) \mathrm{pm}$ $\gamma=84.197(8)^{\circ}$

$0.99429(16) \mathrm{nm}^{3}$

2

$1.198 \mathrm{Mg} / \mathrm{m}^{3}$

$1.626 \mathrm{~mm}^{-1}$

376

$0.50 \times 0.10 \times 0.10 \mathrm{~mm}^{3}$

$1.96-24.74^{\circ}$

$-7<=\mathrm{h}<=7,-12<=\mathrm{k}<=10,-17<=\mathrm{l}<=17$

13096

$3381[\mathrm{R}(\mathrm{int})=0.0781]$

$99.5 \%$

0.8543 und 0.4969

Full-matrix least-squares on $\mathrm{F}^{2}$

$3381 / 0 / 172$

1.008

$\mathrm{R} 1=0.0377, \mathrm{wR} 2=0.0814$

$\mathrm{R} 1=0.0530, \mathrm{wR} 2=0.0860$

0.481 und -0.560 e. $\AA^{-3}$ 


\subsection{Verbindung}

Summenformel

Molare Masse

Temperatur

Wellenlänge

Kristallsystem

Raumgruppe

Zelldimensionen

Zellvolumen

Formeleinheiten pro Zelle

Berechnete Dichte

Absorptionskoeffitient

F (000)

Kristallgröße

Gemessener $\theta$-Bereich

Indexbereich

Anzahl der gemessenen Reflexe

Unabhängige Reflexe

Vollständigkeit an $\theta=22.54^{\circ}$

Max. und min. Transmission

Verfeinerungsmethode

Daten / Restraints / Parameter

Goodness-of-Fit an $\mathrm{F}^{2}$

Endgültige R-Werte [I > $2 \sigma$ (I)]

R-Werte (sämtliche Daten)

Größtes Maximum und Minimum

\section{$\mathrm{H}_{3} \mathrm{Ga} \cdot \mathrm{C}(\mathrm{N}(\text { iso-propyl }))_{2} \mathrm{C}_{2} \mathbf{H}_{2}$}

$\mathrm{C}_{9} \mathrm{H}_{19} \mathrm{GaN}_{2}$

224.98

133(2) K

$71.073 \mathrm{pm}$

orthorhombisch

Pca2(1)

$$
\begin{array}{ll}
\mathrm{a}=1181.16(12) \mathrm{pm} & \alpha=90^{\circ} \\
\mathrm{b}=1067.24(12) \mathrm{pm} & \beta=90^{\circ} \\
\mathrm{c}=1895.0(2) \mathrm{pm} & \gamma=90^{\circ}
\end{array}
$$

$2.3888(4) \mathrm{nm}^{3}$

8

$1.251 \mathrm{Mg} / \mathrm{m}^{3}$

$2.262 \mathrm{~mm}^{-1}$

944

$0.30 \times 0.20 \times 0.20 \mathrm{~mm}^{3}$

$1.91-26.96^{\circ}$

$-11<=\mathrm{h}<=12,-12<=\mathrm{k}<=13,-19<=\mathrm{l}<=22$

6879

$3765[\mathrm{R}(\mathrm{int})=0.0299]$

$77.6 \%$

0.6604 und 0.5502

Full-matrix least-squares on $\mathrm{F}^{2}$

3765 / $16 / 249$

1.023

$\mathrm{R} 1=0.0289, \mathrm{wR} 2=0.0761$

$\mathrm{R} 1=0.0319, \mathrm{wR} 2=0.0780$

0.492 und -0.375 e. $\AA^{-3}$ 


\subsection{Verbindung}

Summenformel

Molare Masse

Temperatur

Wellenlänge

Kristallsystem

Raumgruppe

Zelldimensionen

Zellvolumen

Formeleinheiten pro Zelle

Berechnete Dichte

Absorptionskoeffitient

F (000)

Kristallgröße

Gemessener $\theta$-Bereich

Indexbereich

Anzahl der gemessenen Reflexe

Unabhängige Reflexe

Vollständigkeit an $\theta=22.54^{\circ}$

Max. und min. Transmission

Verfeinerungsmethode

Daten / Restraints / Parameter

Goodness-of-Fit an $\mathrm{F}^{2}$

Endgültige R-Werte [I $>2 \sigma(\mathrm{I})]$

R-Werte (sämtliche Daten)

Größtes Maximum und Minimum

\section{$\mathrm{I}_{3} \mathrm{Ga} \cdot \mathrm{C}(\mathrm{NMes})_{2} \mathrm{C}_{2} \mathrm{H}_{2}$}

$\mathrm{C}_{28} \mathrm{H}_{32} \mathrm{GaI}_{3} \mathrm{~N}_{2}$

846.98

200(2) K

$71.073 \mathrm{pm}$

orthorhombisch

Pnma

$\mathrm{a}=1464.5(3) \mathrm{pm}$

$\alpha=90^{\circ}$

$\mathrm{b}=2429.8(9) \mathrm{pm}$

$\beta=90^{\circ}$

$\mathrm{c}=869.45(17) \mathrm{pm}$

$\gamma=90^{\circ}$

$3.0938(14) \mathrm{nm}^{3}$

4

$1.818 \mathrm{Mg} / \mathrm{m}^{3}$

$3.901 \mathrm{~mm}^{-1}$

1616

$0.70 \times 0.60 \times 0.60 \mathrm{~mm}^{3}$

$3.64-24.96^{\circ}$

$-17<=\mathrm{h}<=17,-28<=\mathrm{k}<=28,-10<=\mathrm{l}<=10$

5560

$2780[\mathrm{R}(\mathrm{int})=0.0912]$

$99.6 \%$

0.2031 und 0.1710

Full-matrix least-squares on $\mathrm{F}^{2}$

$2780 / 286 / 178$

1.125

$\mathrm{R} 1=0.0561, \mathrm{wR} 2=0.1485$

$\mathrm{R} 1=0.0608, \mathrm{wR} 2=0.1537$

1.503 und -1.574 e. $\AA^{-3}$ 


\subsection{Verbindung}

Summenformel

Molare Masse

Temperatur

Wellenlänge

Kristallsystem

Raumgruppe

Zelldimensionen

Zellvolumen

Formeleinheiten pro Zelle

Berechnete Dichte

Absorptionskoeffitient

F (000)

Kristallgröße

Gemessener $\theta$-Bereich

Indexbereich

Anzahl der gemessenen Reflexe

Unabhängige Reflexe

Vollständigkeit an $\theta=22.54^{\circ}$

Max. und min. Transmission

Verfeinerungsmethode

Daten / Restraints / Parameter

Goodness-of-Fit an $\mathrm{F}^{2}$

Endgültige R-Werte [I > $2 \sigma$ (I)]

R-Werte (sämtliche Daten)

Größtes Maximum und Minimum

\section{(Dipp) ${ }_{2} \mathrm{NacNacGaBr}_{2}$}

$\mathrm{C}_{29} \mathrm{H}_{41} \mathrm{Br}_{2} \mathrm{GaN}_{2}$

647.18

133(2) K

$71.073 \mathrm{pm}$

monoklin

$\mathrm{P} 2(1) / \mathrm{n}$

$\mathrm{a}=1268.51(15) \mathrm{pm}$

$\alpha=90^{\circ}$

$\mathrm{b}=1972.9(2) \mathrm{pm}$

$\beta=117.426(9)^{\circ}$

$\mathrm{c}=1332.12(19) \mathrm{pm}$

$\gamma=90^{\circ}$
$2.9591(6) \mathrm{nm}^{3}$

4

$1.453 \mathrm{Mg} / \mathrm{m}^{3}$

$3.650 \mathrm{~mm}^{-1}$

1320

$0.30 \times 0.30 \times 0.20 \mathrm{~mm}^{3}$

$1.84-24.84^{\circ}$

$-14<=\mathrm{h}<=14,-23<=\mathrm{k}<=21,-15<=\mathrm{l}<=15$

16490

$5068[\mathrm{R}(\mathrm{int})=0.0707]$

$99.1 \%$

0.5289 und 0.4073

Full-matrix least-squares on $\mathrm{F}^{2}$

5068 / 0 / 317

0.950

$\mathrm{R} 1=0.0362, \mathrm{wR} 2=0.0926$

$\mathrm{R} 1=0.0424, \mathrm{wR} 2=0.0962$

1.297 und -1.037 e. $\AA^{-3}$ 


\subsection{Verbindung}

Summenformel

Molare Masse

Temperatur

Wellenlänge

Kristallsystem

Raumgruppe

Zelldimensionen

Zellvolumen

Formeleinheiten pro Zelle

Berechnete Dichte

Absorptionskoeffitient

F (000)

Kristallgröße

Gemessener $\theta$-Bereich

Indexbereich

Anzahl der gemessenen Reflexe

Unabhängige Reflexe

Vollständigkeit an $\theta=22.54^{\circ}$

Max. und min. Transmission

Verfeinerungsmethode

Daten / Restraints / Parameter

Goodness-of-Fit an $\mathrm{F}^{2}$

Endgültige R-Werte [I $>2 \sigma(\mathrm{I})]$

R-Werte (sämtliche Daten)

Größtes Maximum und Minimum

\section{(Dipp) ${ }_{2} \mathrm{NacNacGaF}_{2}$}

$\mathrm{C}_{29} \mathrm{H}_{41} \mathrm{~F}_{2} \mathrm{GaN}_{2}$

525.36

133(2) K

$71.073 \mathrm{pm}$

monoklin

$\mathrm{P} 2(1) / \mathrm{n}$

$\mathrm{a}=1250.03(8) \mathrm{pm}$

$\alpha=90^{\circ}$

$\mathrm{b}=1586.89(6) \mathrm{pm}$

$\beta=104.495(5)^{\circ}$

$\mathrm{c}=1428.49(7) \mathrm{pm}$ $\gamma=90^{\circ}$
$2.7434(2) \mathrm{nm}^{3}$

4

$1.272 \mathrm{Mg} / \mathrm{m}^{3}$

$1.035 \mathrm{~mm}^{-1}$

1112

$0.20 \times 0.20 \times 0.20 \mathrm{~mm}^{3}$

$1.94-24.82^{\circ}$

$-14<=\mathrm{h}<=14,-18<=\mathrm{k}<=18,-16<=\mathrm{l}<=16$

40901

$4709[\mathrm{R}(\mathrm{int})=0.0688]$

$99.7 \%$

0.8197 und 0.8197

Full-matrix least-squares on $\mathrm{F}^{2}$

4709 / 0 / 317

0.951

$\mathrm{R} 1=0.0334, \mathrm{wR} 2=0.0701$

$\mathrm{R} 1=0.0539, \mathrm{wR} 2=0.0746$

0.413 und -0.377 e. $\AA^{-3}$ 


\subsection{Verbindung}

Summenformel

Molare Masse

Temperatur

Wellenlänge

Kristallsystem

Raumgruppe

Zelldimensionen

Zellvolumen

Formeleinheiten pro Zelle

Berechnete Dichte

Absorptionskoeffitient

F (000)

Kristallgröße

Gemessener $\theta$-Bereich

Indexbereich

Anzahl der gemessenen Reflexe

Unabhängige Reflexe

Vollständigkeit an $\theta=22.54^{\circ}$

Max. und min. Transmission

Verfeinerungsmethode

Daten / Restraints / Parameter

Goodness-of-Fit an $\mathrm{F}^{2}$

Endgültige R-Werte [I > $2 \sigma$ (I)]

R-Werte (sämtliche Daten)

Größtes Maximum und Minimum

\section{(Dipp) ${ }_{2} \mathrm{NacNacGaH}$}

$\mathrm{C}_{29} \mathrm{H}_{43} \mathrm{GaN}_{2}$

489.37

133(2) K

$71.073 \mathrm{pm}$

monoklin

$\mathrm{P} 2(1) / \mathrm{n}$

$\mathrm{a}=1250.80(7) \mathrm{pm}$

$\alpha=90^{\circ}$

$\mathrm{b}=1618.84(6) \mathrm{pm}$

$\beta=105.055(4)^{\circ}$

$\mathrm{c}=1409.49(7) \mathrm{pm}$

$\gamma=90^{\circ}$
$2.7560(2) \mathrm{nm}^{3}$

4

$1.179 \mathrm{Mg} / \mathrm{m}^{3}$

$1.016 \mathrm{~mm}^{-1}$

1048

$0.30 \times 0.30 \times 0.20 \mathrm{~mm}^{3}$

$1.96-24.79^{\circ}$

$-14<=\mathrm{h}<=14,-19<=\mathrm{k}<=18,-16<=\mathrm{l}<=16$

38900

$4716[\mathrm{R}(\mathrm{int})=0.0630]$

$99.6 \%$

0.8226 und 0.7503

Full-matrix least-squares on $\mathrm{F}^{2}$

4716 / 0 / 307

1.033

$\mathrm{R} 1=0.0292, \mathrm{wR} 2=0.0668$

$\mathrm{R} 1=0.0390, \mathrm{wR} 2=0.0697$

0.223 und -0.343 e. $\AA^{-3}$ 


\subsection{Verbindung}

Summenformel

Molare Masse

Temperatur

Wellenlänge

Kristallsystem

Raumgruppe

Zelldimensionen

Zellvolumen

Formeleinheiten pro Zelle

Berechnete Dichte

Absorptionskoeffitient

F (000)

Kristallgröße

Gemessener $\theta$-Bereich

Indexbereich

Anzahl der gemessenen Reflexe

Unabhängige Reflexe

Vollständigkeit an $\theta=22.54^{\circ}$

Max. und min. Transmission

Verfeinerungsmethode

Daten / Restraints / Parameter

Goodness-of-Fit an $\mathrm{F}^{2}$

Endgültige R-Werte [I > $2 \sigma(\mathrm{I})]$

R-Werte (sämtliche Daten)

Größtes Maximum und Minimum

\section{$\left[(\text { Dipp })_{2} \mathrm{NacNacH}_{2}\right]^{+}\left[\mathrm{OOCCF}_{3}\right]^{-} \cdot \mathrm{OEt}_{2}$}

$\mathrm{C}_{35} \mathrm{H}_{53} \mathrm{~F}_{3} \mathrm{~N}_{2} \mathrm{O}_{3}$

606.79

133(2) K

$71.073 \mathrm{pm}$

monoklin

$\mathrm{P} 2(1) / \mathrm{n}$

$$
\begin{array}{ll}
\mathrm{a}=1716.35(11) \mathrm{pm} & \alpha=90^{\circ} \\
\mathrm{b}=1291.88(7) \mathrm{pm} & \beta=117.301(4)^{\circ} \\
\mathrm{c}=1798.87(11) \mathrm{pm} & \gamma=90^{\circ}
\end{array}
$$

\section{$3.5444(4) \mathrm{nm}^{3}$}

4

\section{$1.137 \mathrm{Mg} / \mathrm{m}^{3}$}

$0.082 \mathrm{~mm}^{-1}$

1312

$0.20 \times 0.20 \times 0.10 \mathrm{~mm}^{3}$

$2.03-24.81^{\circ}$

$-20<=\mathrm{h}<=20,-15<=\mathrm{k}<=15,-21<=\mathrm{l}<=21$

35819

$6092[\mathrm{R}(\mathrm{int})=0.0877]$

$99.5 \%$

0.9918 und 0.9838

Full-matrix least-squares on $\mathrm{F}^{2}$

6092 / 0 / 401

1.011

$\mathrm{R} 1=0.0574, \mathrm{wR} 2=0.1371$

$\mathrm{R} 1=0.0974, \mathrm{wR} 2=0.1537$

0.600 und -0.493 e. $\AA^{-3}$ 


\subsection{Literaturverzeichnis}

[1] http://de.wikipedia.org/wiki/Gallium-Arsenid

[2] http://www.it2006.de/kapitel4_3_4.html

[3] Ashby, E. C., Adv. Inorg. Radiochem. 1966, 8, 283-335.

[4] Wiberg, E.; Amberger, E.: „Hydrides“, Elsevier, Amsterdam, 1971, S. 443454.

[5] Shirk, A. E.; Shriver, D. F., Inorg. Synth. 1977, 17, 45-47.

[6] Shriver, D. F.; Shirk, A. E., Inorg. Synth. 1977, 17, 42-45.

[7] Uhl, W.; Breher, F.; Lützen, A.; Saak, W., Angew. Chem. 2000, 112, 414.

[8] Uhl, W.; Breher, F.; Grunnenberg, J.; Lützen, A.; Saak, W., Organometallics 2000, 19, 4536.

[9] Uhl, W.; Breher, F.; Mbonimana, A.; Gauss, J.; Haase, D.; Lützen, A.; Saak, W., J. Inorg. Chem. 2001, 3059.

[10] Schiefer, M.; Reddy, N. D., Ahn, H.-J.; Stasch, A.; Roesky, H. W.; Schlicker, A. C.; Schmidt, H.-G.; Noltemeyer, M.; Vidovic, D., Inorg. Chem. 2003, 42, 4970.

[11] Uhl, W.; Breher, F.; Haddadpour, S.; Rogel, F., Organometallics 2005, 24, 2210-2213.

[12] Uhl, W.; Cuypers, L.; Neumueller, B.; Weller, F., Organometallics 2002, 21, $2365-2368$.

[13] Novikova, O. A.; Kuznetsova, V. P.; Kornev, K. A., Zhurnal Obshchei Khimii 1968, $38,178-180$.

[14] Kruerke, U., J. Organomet. Chem. 1970, 21, 83-90.

[15] Komarov, N. V.; Loi, L. I., Zhurnal Obshchei Khimii 1975, 45, 2101. 
[16] Stender, M.; Eichler,B. E.; Hardman, N. J.; Power, P. P.; Prust, J.; Noltemeyer, M.; Roesky, H. W., Inorg. Chem. 2001, 40, 2794-2799.

[17] Herzog, A.; Liu, F.-Q.; Roesky, H. W.; Demsar, A.; Keller, K.; Noltemeyer, M.; Pauer, F., Organometallics 1994, 13, 1251-1256.

[18] Murphy, E. F.; Luebben, T.; Herzog, A.; Roesky, H. W.; Demsar, A.; Noltemeyer, M.; Schmidt, H.-G., Inorg. Chem. 1996, 35, 23-29.

[19] Liu, F.-Q.; Herzog, A.; Roesky, H. W.; Uson, I., Inorg. Chem. 1996, 35, $741-744$.

[20] Herzog, A.; Roesky, H. W.; Jaeger, F.; Steiner, A., Chem. Commun. 1996, 1, 29-30.

[21] Twamley, B.; Hardman, N. J; Power, P. P., Acta Crystallo., Sect. E: Structure Reports Online 2001, E57, m227-m228.

[22] Roesky, H. W.; Herzog, A.; Keller, K., Z. Naturforsch., B: Chem. Sciences 1994, 49, 981-982.

[23] Cui, C.; Roesky, H. W.; Hao, H.; Schmidt, H.-G.; Noltemeyer, M., Ang. Chem., Int. Ed. 2000, 39, 1815-1817.

[24] Hardman, N. J.; Eichler, B. E.; Power, P. P., Chem. Commun. 2000, 20, 1991-1992.

[25] Clegg, W.; Cope, E. K.; Edwards, A. J.; Mair, F. S., Inorg. Chem. 1998, 37, 2317-2319.

[26] Autorenkollektiv, Organikum, 19. Aufl., VEB Deutscher Verlag der Wissenschaften, Berlin, 1993.

[27] Kottke, T.; Stalke, D., J. Appl. Crystallogr. 1993, 26, 615.

[28] Sheldrick, G. M., Acta Crystallogr. 1990, A 46, 467. 
[29] SHELXL, Programm zur Kristallstruktur-Verfeinerung, Sheldrick, G. M., Universität Göttingen, 1997.

[30] Starikova, O. V.; Dolgushin, G. V.; Larina, L. I.; Komarova, T. N.; Lopyrev, V. A., ARKIVOC (Gainesville, FL, United States) 2003, 13, 119-124.

[31] Arduengo, A. J., III; Krafczyk, R.; Schmutzler, R.; Craig, H. A.; Goerlich, J. R.; Marshall, W. J.; Unverzagt, M., Tetrahedron 1999, 55, 14523-14534.

[32] Sauvetre, R.; Normant, J.; Villiras, J., Tetrahedron 1975, 31, 897-903.

[33] Waycole, L.; Paquette, L. A., Organic Syntheses 1989, 67, 149-156.

[34] Agliukov, N. Kh.; Nikolaeva, L. G., Trudy po Khimii i Khimicheskoi Tekhnologii 1973, 4, 49-50.

[35] Kovar, R. A., Inorg. Synt. 1977, 17 167-172.

[36] Breusov, O. N.; Lavrent'eva, V. G., Metody Polucheniya Khimicheskikh Reaktivov i Preparatov 1967, 16 130-131.

[37] Gil, F. J. M.; Salgado, M. A.; Gil, J. M., Synth. and React. Inorg. MetalOrganic Chem. 1986, 16, 663-666.

[38] Stender, M.; Wright, R. J.; Eichler, B. E.; Prust, J.; Olmstead, M. M.; Roesky, H. W.; Power, P. P., J. Chem. Soc, Dalt. Trans. 2001, 23, 3465-3469.

[39] Song, L.; Zhao, Z.; Wang, J., Huaxue Shiji 1986, 8, 104-105.

[40] Brown, H. C.; Kramer, G. W.; Hubbard, J. L.; Krishnamurthy, S., J. Organomet. Chem. 1980, 188, 1-10.

[41] Wood, M. E., Science of Synthesis 2003, 5, 315-352. 


\section{Lebenslauf:}

$\begin{array}{lcl}\text { Name } & : & \text { Ahn } \\ \text { Geburtsname } & : & \text { Schuster } \\ \text { Vorname } & : & \text { Hans-Jürgen } \\ \text { Geburtsort } & : & \text { Erfurt } \\ \text { Geburtsdatum } & : & 17.07 .1968 \\ \text { Adresse } & : & \text { Erich-Schmidt-Weg 1, 37083 Göttingen } \\ \text { Tel. } & : & \text { 0551/5030571 } \\ \text { Eltern } & : & \text { Siegfried Schuster (Ingenieur) } \\ & : & \begin{array}{l}\text { Christine Schuster, geb.Walter } \\ \text { (Krankenschwester) }\end{array} \\ \text { Familienstand } & : & \begin{array}{l}\text { verheiratet (seit 07.07.2001 mit Soo-Hyun Ahn) } \\ \text { Kinder }\end{array} \\ \text { Staatsangehörigkeit : } & : & \begin{array}{l}\text { Aaron Jae-Sun Ahn, geboren am 02.03.2004 } \\ \text { deutsch }\end{array}\end{array}$

\section{schulische Ausbildung:}

$\begin{array}{ll}1975-1977 & \text { Pestalozzi-Oberschule Erfurt } \\ 1977-1978 & \text { Hochheimer-Oberschule Erfurt } \\ 1978-1982 & \text { Maxim-Gorki-Oberschule Dermbach } \\ 1982-1984 & \text { Geschwister-Scholl-Oberschule Dachwig } \\ 04.1984 & \text { Übersiedlung von der „DDR“ in die Bundesrepublik } \\ & \text { Deutschland } \\ 1984 & \text { Gesamtschule-Ahnatal Vellmar } \\ 1984-1985 & \text { Goethe-Gymnasium I Kassel } \\ 1985-1988 & \text { Goethe-Gymnasium II Kassel } \\ 06.1988 & \text { Abitur }\end{array}$

\section{Bundeswehr:}

10. -12.1988

Grundausbildung in Koblenz

01. -12.1989

Stammeinheit in Homberg/Efze

\section{universitäre Ausbildung:}

10.1989

Immatrikulation an der Georg-August-Universität

Göttingen

07.1996 Diplomvorprüfung in Chemie

07.1999 - 03.2000 Diplomarbeit unter der Leitung von Prof. Dr. Dr.

h.c.mult. H. W. Roesky am Institut für Anorganische

Chemie der Georg-August-Universität Göttingen

05.2000

seit 05.2000

Diplomprüfung in Chemie

Promotion unter der Leitung von Prof. Dr. Dr. h.c.mult. H. W. Roesky am Institut für Anorganische Chemie der Georg-August-Universität

Göttingen 


\section{Publikationsliste}

[1] Schiefer, M.; Reddy, N. D.; Ahn, H.-J.; Stasch, A.; Roesky, H. W.; Schlicker, A. C.; Schmidt, H.-G.; Noltemeyer, M.; Vidovic, D., Neutral and ionic aluminum, gallium, and indium compounds carrying two or three terminal ethynyl groups, Inorg. Chem. 2003, 42, 4970.

[2] Singh, S.; Kumar, S. S.; Chandrasekhar, V.; Ahn, H.-J.; Biadene, M.; Roesky, H. W.; Hosmane, N. S.; Noltemeyer, M.; Schmidt, H.-G., Tetranuclear Homo- and Heteroalumoxanes Containing Reactive Functional Groups: Syntheses and X-ray Crystal Structures of $\left[\left\{[\mathrm{LAl}(\mathrm{Me})](\mu-\mathrm{O})\left(\mathrm{MH}_{2}\right)\right\}_{2}\right]$, Ang. Chem., Int. Ed. 2004, 43, 4940-4943.

[3] Singh, S.; Ahn, H.-J.; Stasch, A.; Jancik, V.; Roesky, H. W.; Pal, A.; Biadene, M.; Herbst-Irmer, R.; Noltemeyer, M.; Schmidt, H.-G., Syntheses, Characterization and X-ray Crystal Structures of $\beta$-diketiminate Group 13 Hydrides, Chlorides and Fluorides, Inorg. Chem. 2006, 45, 1853-1860. 